\title{
GENERALIZED LINEAR MODELS WITH NONIGNORABLE MISSING COVARIATES
}

\author{
by
}

\section{Sonia Afroje Lopa}

A thesis submitted to the Faculty of Graduate and Postdoctoral Affairs in partial fulfilment of the requirements for the degree of

\section{MASTER OF SCIENCE}

IN

Probability And Statistics

Carleton University

OtTaWA, Ontario

(C) 2017

Sonia Afroje Lopa 


\section{Abstract}

In this thesis, we present an overview of generalized linear models (GLMs) for binary and count data with missing covariates when the missing data mechanism is nonignorable. We study the maximum likelihood method to estimate the parameters in GLMs. Particularly, we consider joint estimation of the regression parameters and nuisance parameters by the maximum likelihood method, when some covariates are nonignorably missing. We study a set of estimating equations from the maximum likelihood method for fitting regression models to binary and Poisson data in the presence of missing covariates.

Simulations were carried out to observe the behaviour of the maximum likelihood estimates under both correctly specified and misspecified structures. Our simulation study shows that if the fitted model is correctly specified, then the maximum likelihood method generally provides unbiased and efficient estimators, where as a misspecified model provides biased and inefficient estimators. The simulation results also indicate that when the sample size is small, the empirical coverage probabilities of the parameter estimates are a bit apart from the nominal 95\% level. But they tend to get closer to the nominal level when the sample size is larger. Also, the average lengths of the confidence intervals for the regression parameters tend to be smaller for larger sample size, as expected. Under misspecified missing data models, we observe systemic bias in the regression estimators and also poor coverage probabilities from the confidence intervals.

We conclude that when analyzing incomplete data with missing covariates, it is necessary to incorporate a suitable missing data model into the observed data likelihood function in order to obtain unbiased and efficient estimators of the model parameters. We also note that a misspecified missing data model can provide systematic bias in the maximum likelihood estimation. So it is important to assess the validity of a missing 
data model when performing a likelihood inference based on the given observed data. 


\section{Acknowledgements}

I would like to express my deepest gratitude to my supervisor, Dr. Sanjoy K. Sinha for his kind supervision, advice, and guidance. It has been an honour to get a chance of being his M.Sc. student. I appreciate all his encouragement, productive ideas, and support throughout this work. Completion of this work would have been impossible without his guidance.

I also thank many people in the School of Mathematics and Statistics at Carleton University for their help, continual support to continue my graduate study in Statistics. Specially I would like to thank Nicole Gaertner, the Graduate Administrator of the school, for her valuable help and suggestions.

I am grateful to Alia Alkhathami and all friends in my group specially Parvin Dehghani, Marie-Joe Nemnom, Touba Warsi for sharing their experience and knowledge during my graduate study.

It is an honour to thank my wonderful mother Shahera Akter who has dedicated her whole life to my sisters and myself and provided unconditional love and care. Also, she has helped me a lot in this very crucial time by looking after my first baby girl. I would like to extend my sincere thanks to my two sisters Lovely Akter and Lata Akter for their love, support, and encouragement in the higher education from my childhood. And most of all my loving, caring, encouraging and patient husband Musharraf whose support in every stage of this M.Sc is unforgettable. I also want to remember my nephew (Soad), nieces (Aboni, Tanha, Abriti) and my new born daughter Aritri (Mahdisha Afroje) who have enriched my life with joy and happiness.

Lastly, I offer my regards and blessing to all of those who supported me in any aspect 
during the completion of my study. I am heartily thankful to all of you. 
I dedicate this thesis to My Daughter And My Mother Mahdisha Afroje Aritri And Shahera Akter for their unconditional love. 


\section{Contents}

Abstract

Acknowledgements $\quad$ iii

Abbreviations xiii

1 Introduction $\quad 1$

1.1 Generalized Linear Model . . . . . . . . . . . . . . . . . . . . . . . . 1

1.2 Missing Data Problems . . . . . . . . . . . . . . . . . . . 2

1.3 Statement of the Problems . . . . . . . . . . . . . . . . 5

1.4 Organization of the Thesis . . . . . . . . . . . . . . 6

2 Generalized Linear Models And Missing Covariates $\quad 7$

2.1 Introduction . . . . . . . . . . . . . . . . . . 7

2.2 Linear Models . . . . . . . . . . . . . . . . . . . . . . 7

2.3 Literature on Generalized Linear Models . . . . . . . . . . . . . . . . . . 9

2.3.1 Structure of GLMs . . . . . . . . . . . . . . . . . . . . . . . . 10

2.3.2 Maximum Likelihood Estimation in GLMs . . . . . . . . . . . . . 12

2.4 Literature on Missing Data . . . . . . . . . . . . . . . . . . . 15

2.5 Literature on Missing Covariates . . . . . . . . . . . . . . . 16

3 Maximum Likelihood Estimation under Nonignorible Missing Covari$\begin{array}{ll}\text { ates } & 20\end{array}$

3.1 Introduction . . . . . . . . . . . . . . . . . . . 20

3.2 Model and Notation . . . . . . . . . . . . . . . . . . . 20

3.2.1 Full Model . . . . . . . . . . . . . . . . . . . . . . . 20

3.2.2 Model for Covariates . . . . . . . . . . . . . . . . . . . 21 
3.2 .3 Model for Missing Data . . . . . . . . . . . . . . . 23

3.3 Methods of Estimation . . . . . . . . . . . . . . . . . . 25

3.3.1 ML Estimation under Missing Covariates . . . . . . . . . . . . 25

3.3.2 Asymptotic Variance of the ML Estimator . . . . . . . . . . . 28

3.3.3 Newton-Raphson Method . . . . . . . . . . . . . . . . 29

3.3 .4 Asymptotics . . . . . . . . . . . . . . . . . 30

4 Binary Regression $\quad 34$

4.1 Introduction . . . . . . . . . . . . . . . . 34

4.2 Model and Notation . . . . . . . . . . . . . . . . . 35

4.2 .1 Binary Logistic Model . . . . . . . . . . . . . . . . 35

4.2 .2 Maximum Likelihood Estimation . . . . . . . . . . . . . 38

4.3 Simulation Study . . . . . . . . . . . . . . . . . . . . . . . . . 39

4.3 .1 Investigative Methods . . . . . . . . . . . . . . . . . . 40

4.3.2 Binary Logistic Model for Simulation . . . . . . . . . . . . . . 42

4.3.3 Results and Discussion for the Binary Model . . . . . . . . . . . . 44

5 Poisson Regression $\quad 63$

5.1 Introduction . . . . . . . . . . . . . . . . . 63

5.2 Model and Notation . . . . . . . . . . . . . . . . . . 65

5.2 .1 Poisson Model for Count Data . . . . . . . . . . . . . . . 65

5.2 .2 Maximum Likelihood Estimation . . . . . . . . . . . . 66

5.3 Simulation Study . . . . . . . . . . . . . . . . . . 67

5.3 .1 Poisson Model for Simulation . . . . . . . . . . . . . 68

5.3.2 Results and Discussion for the Poisson Model . . . . . . . . . . 69

$\begin{array}{llr}6 & \text { Conclusion } & 87\end{array}$

6.1 Future Research . . . . . . . . . . . . . . . . . . . . . . . . . 88

$\begin{array}{ll}\text { Bibliography } & 89\end{array}$

$\begin{array}{ll}\text { A R Codes For Binary Data } & 93\end{array}$

B R Codes For Count Data 110 


\section{List of Tables}

4.1 Empirical biases and mean squared errors (MSEs) of maximum likelihood estimators in binary logistic regression models with missing covariates for different sample sizes. Slope parameter $\beta_{1}=1$. Correctly specified MAR model is assumed for missing data. . . . . . . . . . . . . . . . 45

4.2 Coverage probabilities (CPs), average lengths of maximum likelihood estimators in binary logistic regression models with missing covariates for different sample sizes. Slope parameter $\beta_{1}=1$. Correctly specified MAR model is assumed for missing data. . . . . . . . . . . . . . . . 46

4.3 Empirical biases and mean squared errors (MSEs) of maximum likelihood estimators in binary logistic regression models with missing covariates for different sample sizes. Slope parameter $\beta_{1}=0.5$. Correctly specified MAR model is assumed for missing data. . . . . . . . . . . . . . . . 46

4.4 Coverage probabilities (CPs), average lengths of maximum likelihood estimators in binary logistic regression models with missing covariates for different sample sizes. Slope parameter $\beta_{1}=0.5$. Correctly specified MAR model is assumed for missing data. . . . . . . . . . . . . . . . 47

4.5 Empirical biases and mean squared errors (MSEs) of maximum likelihood estimators in binary logistic regression models with missing covariates for different sample sizes. Slope parameter $\beta_{1}=1$. Correctly specified NMAR model is assumed for missing data. . . . . . . . . . . . . . . . . 49

4.6 Coverage probabilities (CPs), average lengths of maximum likelihood estimators in binary logistic regression models with missing covariates for different sample sizes. Slope parameter $\beta_{1}=1$. Correctly specified NMAR model is assumed for missing data. . . . . . . . . . . . . . . . 50 
4.7 Empirical biases and mean squared errors (MSEs) of maximum likelihood estimators in binary logistic regression models with missing covariates for different sample sizes. Slope parameter $\beta_{1}=0.5$. Correctly specified NMAR model is assumed for missing data. . . . . . . . . . . . . . . . . . 51

4.8 Coverage probabilities (CPs), average lengths of maximum likelihood estimators in binary logistic regression models with missing covariates for different sample sizes. Slope parameter $\beta_{1}=0.5$. Correctly specified NMAR model is assumed for missing data. . . . . . . . . . . . . . . . . 52

4.9 Empirical biases and mean squared errors (MSEs) of maximum likelihood estimators in binary logistic regression models with missing covariates for different sample sizes. Slope parameter $\beta_{1}=1$. Misspecified NMAR model is assumed for missing data. . . . . . . . . . . . . . . . . . 53

4.10 Coverage probabilities (CPs), average lengths of maximum likelihood estimators in binary logistic regression models with missing covariates for different sample sizes. Slope parameter $\beta_{1}=1$. Misspecified NMAR model is assumed for missing data. . . . . . . . . . . . . . . . . . . . . 54

4.11 Empirical biases and mean squared errors (MSEs) of maximum likelihood estimators in binary logistic regression models with missing covariates for different sample sizes. Slope parameter $\beta_{1}=0.5$. Misspecified NMAR model is assumed for missing data. . . . . . . . . . . . . . . . . . . . 55

4.12 Coverage probabilities (CPs), average lengths of maximum likelihood estimators in binary logistic regression models with missing covariates for different sample sizes. Slope parameter $\beta_{1}=0.5$. Misspecified NMAR model is assumed for missing data. . . . . . . . . . . . . . . . . . . 56

4.13 Empirical biases and mean squared errors (MSEs) of maximum likelihood estimators in binary logistic regression models with missing covariates for different sample sizes. Slope parameter $\beta_{1}=1$. Misspecified MAR model is assumed for missing data. . . . . . . . . . . . . . . . . . . . . 57

4.14 Coverage probabilities (CPs), average lengths of maximum likelihood estimators in binary logistic regression models with missing covariates for different sample sizes. Slope parameter $\beta_{1}=1$. Misspecified MAR model is assumed for missing data. . . . . . . . . . . . . . . . . . 58 
4.15 Empirical biases and mean squared errors (MSEs) of maximum likelihood estimators in binary logistic regression models with missing covariates for different sample sizes. Slope parameter $\beta_{1}=0.5$. Misspecified MAR model is assumed for missing data. . . . . . . . . . . . . . . . . 58

4.16 Coverage probabilities (CPs), average lengths of maximum likelihood estimators in binary logistic regression models with missing covariates for different sample sizes. Slope parameter $\beta_{1}=0.5$. Misspecified MAR model is assumed for missing data. . . . . . . . . . . . . . . . . . . . . . 59

5.1 Empirical biases and mean squared errors (MSEs) of maximum likelihood estimators in Poisson regression models with missing covariates for different sample sizes. Slope parameter $\beta_{1}=0.5$. Correctly specified MAR model is assumed for missing data. . . . . . . . . . . . . . . . . 70

5.2 Coverage probabilities (CPs), average lengths of maximum likelihood estimators in Poisson regression models with missing covariates for different sample sizes. Slope parameter $\beta_{1}=0.5$. Correctly specified MAR model is assumed for missing data. . . . . . . . . . . . . . . . . . . . . . . 71

5.3 Empirical biases and mean squared errors (MSEs) of maximum likelihood estimators in Poisson regression models with missing covariates for different sample sizes. Slope parameter $\beta_{1}=1$. Correctly specified MAR model is assumed for missing data. . . . . . . . . . . . . . . . . . . . . 71

5.4 Coverage probabilities (CPs), average lengths of maximum likelihood estimators in Poisson regression models with missing covariates for different sample sizes. Slope parameter $\beta_{1}=1$. Correctly specified MAR model is assumed for missing data. . . . . . . . . . . . . . . . . . . . . . . . 72

5.5 Empirical biases and mean squared errors (MSEs) of maximum likelihood estimators in Poisson regression models with missing covariates for different sample sizes. Slope parameter $\beta_{1}=0.5$. Correctly specified NMAR model is assumed for missing data. . . . . . . . . . . . . . . . . . . . . . 74

5.6 Coverage probabilities (CPs), average lengths of maximum likelihood estimators in Poisson regression models with missing covariates for different sample sizes. Slope parameter $\beta_{1}=0.5$. Correctly specified NMAR model is assumed for missing data. . . . . . . . . . . . . . . . . . . . . . . . . 75 
5.7 Empirical biases and mean squared errors (MSEs) of maximum likelihood estimators in Poisson regression models with missing covariates for different sample sizes. Slope parameter $\beta_{1}=1$. Correctly specified NMAR model is assumed for missing data. . . . . . . . . . . . . . . 76

5.8 Coverage probabilities (CPs), average lengths of maximum likelihood estimators in Poisson regression models with missing covariates for different sample sizes. Slope parameter $\beta_{1}=1$. Correctly specified NMAR model is assumed for missing data. . . . . . . . . . . . . . . . . . . . 76

5.9 Empirical biases and mean squared errors (MSEs) of maximum likelihood estimators in Poisson regression models with missing covariates for different sample sizes. Slope parameter $\beta_{1}=0.5$. Misspecified NMAR model is assumed for missing data. . . . . . . . . . . . . . . . . . . . . 78

5.10 Coverage probabilities (CPs), average lengths of maximum likelihood estimators in Poisson regression models with missing covariates for different sample sizes. Slope parameter $\beta_{1}=0.5$. Misspecified NMAR model is assumed for missing data. . . . . . . . . . . . . . . . . . . . . . . . . 79

5.11 Empirical biases and mean squared errors (MSEs) of maximum likelihood estimators in Poisson regression models with missing covariates for different sample sizes. Slope parameter $\beta_{1}=1$. Misspecified NMAR model is assumed for missing data. . . . . . . . . . . . . . . . . . . . . . 79

5.12 Coverage probabilities (CPs), average lengths of maximum likelihood estimators in Poisson regression models with missing covariates for different sample sizes. Slope parameter $\beta_{1}=1$. Misspecified NMAR model is assumed for missing data. . . . . . . . . . . . . . . . . . . .

5.13 Empirical biases and mean squared errors (MSEs) of maximum likelihood estimators in Poisson regression models with missing covariates for different sample sizes. Slope parameter $\beta_{1}=0.5$. Misspecified MAR model is assumed for missing data. . . . . . . . . . . . . . .

5.14 Coverage probabilities (CPs), average lengths of maximum likelihood estimators in Poisson regression models with missing covariates for different sample sizes. Slope parameter $\beta_{1}=0.5$. Misspecified MAR model is assumed for missing data. . . . . . . . . . . . . . . . . 82 
5.15 Empirical biases and mean squared errors (MSEs) of maximum likelihood estimators in Poisson regression models with missing covariates for different sample sizes. Slope parameter $\beta_{1}=1$. Misspecified MAR model is assumed for missing data. . . . . . . . . . . . . . . . . . . . . 83

5.16 Coverage probabilities (CPs), average lengths of maximum likelihood estimators in Poisson regression models with missing covariates for different sample sizes. Slope parameter $\beta_{1}=1$. Misspecified MAR model is assumed for missing data. . . . . . . . . . . . . . . . . . . . . . 83 


\section{Abbreviations}

$\begin{array}{ll}\text { CI } & \text { Confidence Interval } \\ \text { GLM } & \text { Generalized Linear Model } \\ \text { LM } & \text { Linear Model } \\ \text { MAR } & \text { Missing at Random } \\ \text { MCAR } & \text { Missing Completely at Random } \\ \text { NMAR } & \text { Nonignorable Missing at Random } \\ \text { MLE } & \text { Maximum Likelihood Estimates } \\ \text { MSE } & \text { Mean Squared Error } \\ \text { NI } & \text { Non-Ignorable } \\ \text { NR } & \text { Newton-Raphson }\end{array}$




\section{Chapter 1}

\section{Introduction}

\section{$1.1 \quad$ Generalized Linear Model}

In biostatistics and other areas of applied statistics, analysis of generalized linear models (McCullagh and Nelder, 1989) with missing data is attracting more attention to the researchers in recent years. Missing values in the data could be either ignorable or nonignorable (Ibrahim, Lipsitz and Chen, 1999). An individual's unobserved response is called non-ignorable if it is related to the missing values of the response variable (Little, 1982). If there are non-ignorable missing responses, the distribution of the missing data needs to be correctly modelled to avoid any biases that may be resulted from the incomplete data analysis (Baker and Laird, 1988).

In regression analysis, the generalized linear model (GLM) has been considered one of the most significant advanced methods in the past decades (Hoffman, 2004). GLM is referred to as the most significant statistical tool since it is very flexible to address a variety of statistical problems and has the available software to fit the models. 
There is a substantial confusion because of the abbreviation GLM, some practitioners use a term to refer this to the general linear model, which only comprises LMs, analysis of variance, and analysis of covariance (Lane, 2002). On the other hand, GLMs provide a unified framework, incorporating ordinary general linear models as well as many common nonlinear models (Wu, 2005).

The generalized linear model (GLM) is a common generalization of ordinary linear regression when the error distribution of response variables is not normal including count, binary, proportions and positive valued continuous distributions (Nelder and Wedderburn, 1972; Hilbe, 1994; Hoffman, 2004). Generalized linear models (GLMs) unify different approaches that can handle continuous variables as well as discrete variables when the error distribution of the variables belongs to the natural exponential family such as Poisson and binomial distributions.

One of the approaches to estimating the parameters in GLMs is obtaining the likelihood function by integrating out the random effects from the joint distribution of responses. Usually, Monte Carlo approximations are used to evaluate the integrations since it is quite demanding to evaluate the likelihood function in a closed form (Wei and Tanner, 1990). The likelihood function is maximized to estimate the parameters of interest.

\subsection{Missing Data Problems}

Missing data are common problems that arise very often in biological research. In common phenomenon, an individual's response might not be observed at one follow-up time 
and might be measured at the next follow-up time, resulting in a large number of missingness patterns. When missing data occur, they must be taken into account in order to obtain valid statistical inference.

Missing data patterns are discussed in three different ways according to Little and Rubin (1987), which suggest the relationship between the missingness and observed values in the data. Suppose $Y$ is the complete data that would occur in the absence of missing values. We can write $Y=\left(Y_{\text {obs }}, Y_{\text {mis }}\right)$, where $Y_{\text {obs }}$ denotes the observed values and $Y_{\text {mis }}$ the missing values of $Y$. The density function of the joint distribution of $Y_{\text {obs }}$ and $Y_{\text {mis }}$ can be written as

$$
f(Y \mid \theta) \equiv f\left(Y_{\text {obs }}, Y_{\text {mis }} \mid \theta\right)
$$

depending on some vector of unknown parameters $\theta$.

Consider a binary variable that indicates whether each component of $Y$ is observed or missing. A vector of response indicators $R=\left(R_{i j}\right)$ is defined in such a way that

$$
R_{i j}=\left\{\begin{array}{lll}
1 & \text { if } & y_{i j} \text { is observed } \\
0 & \text { if } \quad y_{i j} \text { is missing }
\end{array}\right.
$$

Here $R$ is treated as a random vector in the model and we assume a joint distribution of $R$ and $Y$. The density of the distribution can be expressed as

$$
f(Y, R \mid \theta, \psi)=f(Y \mid \theta) f(R \mid Y, \psi)
$$


where $f(R \mid Y, \psi)$ depends on some parameters $\psi$, which is the conditional distribution of $R$ given $Y$ for the missing data mechanism.

The data are considered to be missing completely at random (MCAR) when the missing data process does not depend on the observed values as well as the missing values of the data, which means that the missingness is independent of $Y$. It can be expressed as

$$
f\left(R \mid Y_{\mathrm{obs}}, Y_{\mathrm{mis}}, \psi\right)=f(R \mid \psi) .
$$

The missing data mechanism is considered to be missing at random (MAR) when the missingness depends only on the observed values of the variables in the data, which means that the missingness only depends on observed components $Y_{\text {obs }}$ of $Y$. It can be expressed as

$$
f\left(R \mid Y_{\mathrm{obs}}, Y_{\mathrm{mis}}, \psi\right)=f\left(R \mid Y_{\mathrm{obs}}, \psi\right) .
$$

An individual's non-response is considered to be missing not at random (NMAR), when the individual's response probability depends on the response variable itself, which means that the missingness depends on the unobserved values. It can be defined as

$$
f(R, Y \mid X, \theta, \psi)=f(Y \mid X, \theta) f(R \mid Y, X, \psi),
$$


where $X$ represents a full set of covariates. The first component classifies the distribution of $Y$ given $X$ in the population and the second component models the occurrence of response as a function of $Y$ and $X$.

Rubin (1976) discussed that when MAR occurs, the likelihood based inference doesn't depend on missing data mechanism. Baker and Laird (1988) pointed out that if missingness depends on individual's unobserved response, then it is considered to be nonignorable missing data. In such cases, the distribution needed to be modelled for the missing-data mechanism. Several examples that describe the likelihood-based inference using maximum likelihood and missing data mechanisms are discussed in Little (1995)

Likelihood function under certain modelling assumptions is mostly used as an estimation method for missing data. For the ML estimation, the EM algorithm is a well-known iterative algorithm. The M step of the EM algorithm creates a function for the ML estimation when data are complete. The conditional expectation of the current estimates of the parameters for missing data given the observed data is performed on the E step.

\subsection{Statement of the Problems}

In this thesis, we study the maximum likelihood method to make simultaneous inferences for generalized linear models with missing covariates occurring from the nonignorable missing-data mechanism. 
We investigate a set of maximum likelihood equations for fitting regression models to both binary and count data when there are missing values. Under a given missing data mechanism, we study the finite sample properties of empirical biases and mean squared errors as well as coverage probabilities (CPs) and average lengths of the confidence intervals for regression and association parameters for both binary and Poisson models. We also investigate the impact of changing the coefficients of the missing values in terms of biases and mean squared errors as well as coverage probabilities (CPs) and average lengths under both models. Our interest also lies in studying the empirical properties of the parameter estimates in the generalized linear model under both correctly specified and misspecified models.

\subsection{Organization of the Thesis}

The thesis is organized as follows. In Chapter 2, we introduce the generalized linear models (GLMs) and review the literature on missing covariates for analyzing incomplete data. In Chapter 3, we discuss our studied methodology and estimation algorithm based on the maximum likelihood for fitting regression models to binary and Poisson data. In Chapter 4, we present a simulation study and discuss the results of the binary model. We present results from a simulation study for Poisson model in Chapter 5. Chapter 6 concludes the thesis with some discussion and direction for further research. 


\section{Chapter 2}

\section{Generalized Linear Models And Missing Covariates}

\section{$2.1 \quad$ Introduction}

Before we present our methods for estimation in GLMs with nonignorable missing covariates, we give a brief introduction to LMs, GLMs, and models for the missing data in this chapter. In Section 2.2, we introduce LMs and the model. In Section 2.3, we discuss GLMs and methods of estimation for the parameters in GLMs. Section 2.4 presents some reviews of the literature on GLMs. In Section 2.5, we give a literature review of methods for handling non-ignorable missing covariates.

\section{$2.2 \quad$ Linear Models}

Linear models (LMs) are an extensive and venerable issue that provides a rich background of statistical concepts (Janke and Tinsley, 2005). The classical linear models are the most widely used statistical method in regression analysis since they are simple to construct and interpret. They can clarify the linear relationship between the expectation of a response (dependent) variable and a set of explanatory (independent) variables 
very easily.

To estimate the regression coefficients of LMs, the least squares method is mostly used. It provides the most efficient estimator of all unbiased estimators, because it has various suitable statistical properties and thus termed as the "best linear unbiased estimator" or BLUE (Draper and Smith, 1981).

The basic assumption of LMs is the linear relationship between the response variable and the explanatory variables, when values of the response variable (Y) are continuous and follow a conditional normal distribution (given X) with a constant (error) variance.

Let the response variables $\left(Y_{1}, \ldots, Y_{n}\right)$ be independent normal with means $\mu_{i}(i=$ $1,2 \ldots, n)$ and a constant variance $\sigma^{2}$. Suppose $x_{i j}$ is the $j$ th explanatory variable for the $i$ th observation and $\beta_{j}$ is the unknown parameter associated with the $j$ th explanatory variable. Then the classical linear model can be written as

$$
E\left(Y_{i}\right)=\mu_{i}=\sum_{j=1}^{p} \beta_{j} x_{i j}, i=1, \ldots, n .
$$

In matrix notation, it can be rewritten as

$$
E(Y)=\mu=\mathbf{X} \boldsymbol{\beta},
$$

where $\mathbf{X}$ is the $n \times p$ design matrix, $\mu$ is the $n \times 1, \boldsymbol{\beta}$ is the $p \times 1$ vector and $p$ is the number of unknown parameters including the intercept. According to McCullagh and Nelder's (1989) terminology, $Y$ contains the random part, whereas $\mathbf{X} \boldsymbol{\beta}$ is the linear predictor. 


\subsection{Literature on Generalized Linear Models}

The classical linear model is very popular to model a continuous response under certain assumptions. It is more appropriate when the response follows a normal distribution and has a linear relationship with the mean of the covariates. However, sometimes the relationship between the mean of the responses and the covariates cannot be expressed in a linear form. In this case, the standard linear model is unrealistic. Rather than classical linear models, one can consider generalized linear models (GLMs) which generalize the classical linear models, allowing non linear forms between the mean of responses and the covariates.

The generalized linear model for incomplete data is discussed in Ibrahim et al. (1990). A method for estimating parameters in binomial regression models was proposed by Ibrahim and Lipsitz (1996) when the missing data mechanism is non-ignorable. They proposed a conditional model for incomplete covariates in parametric regression models.

In Zhao and Shao (2015), a generalized linear model is considered for identifiability and estimation when the missing data mechanism is nonignorable and unspecified. They assumed a pseudo-likelihood approach by implementing an instrumental variable to support identifying unknown parameters in the presence of nonignorable missing data.

Shi, Zhu, and Ibrahim (2009) developed a method of general local influence to the generalized linear models in the presence of missing covariate data to convey sensitivity analyses of minor perturbations. They also developed various local influence measures 
to identify influential points and test model misspecification. To assess appropriate perturbation schemes, they conducted influence measures based on two objective functions which are the maximum likelihood estimate and the likelihood ratio statistic. Furthermore, they modelled the missing data mechanism as a sequence of one-dimensional conditional distributions of binary logistic regressions.

Sinha (2008) used a generalized linear model for maximum likelihood estimation. He proposed a robust approach to downweight any influential observation in the presence of nonignorable missing covariates. He adopted a Metropolis-Hastings algorithm based on a Markov chain sampling method to carry out some simulations. He examined the behavior of the robust estimates and compared them to the classical maximum likelihood estimates. Finally, he applied his method to real life data of delirium patients.

\subsubsection{Structure of GLMs}

The generalized linear model was defined by Nelder and Wedderburn (1972), which is one of the widely used statistical models considered in regression analysis.

GLMs generalize LMs by relaxing the two assumptions: (i) the response variables are not necessarily to be continuous and to follow normal distributions. They can be categorical or ordinal. (ii) The nonlinear relationship may exist between the responses and explanatory variables.

Let $\left\{\left(y_{i}, \mathbf{x}_{i}\right): i=1, \ldots, n\right\}$ be independent observations, where $y_{i}$ is the $i$ th response variable and $\mathbf{x}_{i}$ is the $i$ th random vector of $p$ covariates. Assume that $y_{i}$ is independently and identically distributed, which follows a distribution in the exponential family. Then 
the density function of each observation $y_{i}$ can be written in the form

$$
f\left(y_{i} ; \boldsymbol{\beta}\right)=\exp \left[\left\{y_{i} \theta_{i}-b\left(\theta_{i}\right)\right\} / a(\phi)+c\left(y_{i}, \phi\right)\right]
$$

for some specific functions $a, b$ and $c$, and $\phi$ is a scaler dispersion parameter (McCullagh and Nelder, 1997). Here, $\theta=\left(\theta_{1}, \theta_{2}, \ldots, \theta_{n}\right)^{t}$ are natural parameters.

Generally, a GLM contains three components including a random component, a systematic component, and a link function.

First, the random components of $y_{i}$ have independent distributions with mean $E\left(y_{i}\right)=$ $\mu_{i}$, which follow a distribution in the exponential family.

Second, the systematic component makes linear relationship between the covariates $\mathbf{x}_{i}$ and the linear predictors $\eta_{i}$ in the form

$$
\eta_{i}=\mathbf{x}_{i}^{t} \boldsymbol{\beta} ; i=1, \ldots, n
$$

where $\boldsymbol{\beta}$ is a vector of regression coefficients.

Third, the link component connects the random components and the systematic components of the model as a form

$$
\eta_{i}=\mathbf{x}_{i}^{t} \boldsymbol{\beta}=g\left(\mu_{i}\right) ; i=1, \ldots, n
$$

Here $g($.$) is a monotonic and differentiable function, connecting the mean and the linear$ predictor. The link function is sometimes invertible and thus is called the inverse link function

$$
g^{-1}\left(\eta_{i}\right)=\mu_{i} ; i=1, \ldots, n
$$

In the exponential family, if a link function $g($.$) satisfies g\left(\mu_{i}\right)=\theta_{i}\left(\mu_{i}\right)$, then the link is called the canonical link. Given an appropriate choice of the link functions, GLMs can 
fit the regression models, where the underlying data may follow the normal, Poisson, or binomial distribution.

\subsubsection{Maximum Likelihood Estimation in GLMs}

The maximum likelihood method is the basic method to estimate the parameters used in GLMs. Assume that the dispersion parameter $\phi$ is known; then $c\left(y_{i}, \phi\right)$ is a constant in the $\log$-likelihood function about $\theta_{i}$. Applying the ML method to GLMs, the loglikelihood function for $n$ independent observations can be written (without the constant term) as

$$
\begin{aligned}
l(\boldsymbol{\theta} \mid \mathbf{y}) & =\sum_{i=1}^{n} \log f\left(y_{i} \mid \theta_{i} ; \phi\right) \\
& =\sum_{i=1}^{n} \frac{y_{i} \theta_{i}-b\left(\theta_{i}\right)}{a(\phi)} .
\end{aligned}
$$

If we take the derivative of Eq.(2.7) with respect to $\theta_{i}$, we get

$$
\frac{\partial l}{\partial \theta_{i}}=\frac{1}{a(\phi)}\left(y_{i}-\frac{\partial b\left(\theta_{i}\right)}{\partial \theta_{i}}\right),
$$

and

$$
\frac{\partial^{2} l}{\partial \theta_{i}{ }^{2}}=-\frac{1}{a(\phi)} \frac{\partial^{2} b\left(\theta_{i}\right)}{\partial \theta_{i}{ }^{2}} .
$$

We take the first derivative of the log-likelihood function with respect to the parameters of interest to obtain the MLEs. To estimate the parameters, we set the derivatives equal to 0 , which are called the likelihood score equations. In general, the likelihood score equation does not have an explicit solution (Firth, 1991). Therefore, to solve the score equation, we often use the iteration method assisted by computer. 
The second derivatives of the log-likelihood function with respect to the parameters of interest $\boldsymbol{\beta}$ provide the variance-covariance matrix of the ML estimator of $\boldsymbol{\beta}$ which is also known as the Fisher information matrix. The asymptotic variance-covariance matrix of the ML estimator $\hat{\boldsymbol{\beta}}$ is the inverse of the Fisher information matrix.

We can show that

$$
\begin{gathered}
E\left(\frac{\partial l}{\partial \theta_{i}}\right)=0 \\
\operatorname{var}\left(\frac{\partial l}{\partial \theta_{i}}\right)=-E\left(\frac{\partial^{2} l}{\partial \theta_{i}^{2}}\right) .
\end{gathered}
$$

Substituting the values of Eq.(2.8) and Eq.(2.9) into Eq.(2.10) and Eq.(2.11), we get

$$
E\left(y_{i}\right)=\mu_{i}\left(\theta_{i}\right)=\frac{\partial b\left(\theta_{i}\right)}{\partial \theta_{i}}
$$

and

$$
\begin{aligned}
\operatorname{var}\left(y_{i}\right) & =\frac{1}{a(\phi)} \frac{\partial^{2} b\left(\theta_{i}\right)}{\partial \theta_{i}^{2}} \\
& =\frac{1}{a(\phi)} \frac{\partial \mu_{i}\left(\theta_{i}\right)}{\partial \theta_{i}} \\
& =\frac{1}{a(\phi)} V\left(\mu_{i}\right)
\end{aligned}
$$

where $V\left(\mu_{i}\right)=\partial \mu_{i}\left(\theta_{i}\right) / \partial \theta_{i}$ is referred to as the variance function. Taking the derivatives on both sides of Eq.(2.5) with respect to $\boldsymbol{\beta}$, we get

$$
\mathbf{x}_{i}=\frac{\partial g\left(\mu_{i}\right)}{\partial \mu_{i}} \frac{\partial \mu_{i}}{\partial \theta_{i}} \frac{\partial \theta_{i}}{\partial \boldsymbol{\beta}}
$$

Also, it can be written as

$$
\frac{\partial \theta_{i}}{\partial \boldsymbol{\beta}}=\frac{1}{V\left(\mu_{i}\right) \partial g\left(\mu_{i}\right) / \partial \mu_{i}} \mathbf{x}_{i}
$$


To get the score function, we differentiate Eq.(2.7) and apply into Eq.(2.12), Eq.(2.13) and Eq.(2.14). Then we obtain the maximum likelihood estimator (MLE) of $\boldsymbol{\beta}$ from the score function

$$
\begin{aligned}
\mathbf{S}(\boldsymbol{\beta}) & =\frac{\partial l(\boldsymbol{\theta} \mid \mathbf{y})}{\partial \boldsymbol{\beta}} \\
& =\sum_{i=1}^{n} \frac{\partial l_{i}\left(\theta_{i} \mid y_{i}\right)}{\partial \theta_{i}} \frac{\partial \theta_{i}}{\partial \boldsymbol{\beta}} \\
& =\frac{1}{a(\phi)} \sum_{i=1}^{n} \frac{y_{i}-\mu_{i}}{V\left(\mu_{i}\right) \partial g\left(\mu_{i}\right) / \partial \mu_{i}} \mathbf{x}_{i} .
\end{aligned}
$$

In matrix form, Eq.(2.15) can be rearranged as

$$
\frac{\partial l}{\partial \boldsymbol{\beta}}=\mathbf{S}(\boldsymbol{\beta})=\frac{1}{a(\phi)} \mathbf{X} W D(\mathbf{Y}-\mu(\boldsymbol{\beta}))
$$

where the design matrix,

$$
\begin{gathered}
\mathbf{X}=\left\{\mathbf{x}_{1}, \mathbf{x}_{2}, \ldots, \mathbf{x}_{n}\right\}, \\
\boldsymbol{\mu}=\left(\mu_{1}, \mu_{2}, \ldots, \mu_{n}\right), \\
W=\operatorname{diag}^{-1}\left\{V\left(\mu_{1}\right)\left(\partial g\left(\mu_{1}\right) / \partial \mu_{1}\right)^{2}, \ldots, V\left(\mu_{n}\right)\left(\partial g\left(\mu_{n}\right) / \partial \mu_{n}\right)^{2}\right\},
\end{gathered}
$$

and

$$
D=\operatorname{diag}\left\{\partial g\left(\mu_{1}\right) / \partial \mu_{1}, \partial g\left(\mu_{2}\right) / \partial \mu_{2} \ldots, \partial g\left(\mu_{n}\right) / \partial \mu_{n}\right\}
$$

To obtain the MLEs, Eq.(2.16) can be solved by performing Fisher scoring algorithm or Gauss-Newton algorithm. Both Fisher scoring and Newton-Raphson methods reduce to the iteratively re-weighted least squares algorithm in the presence of canonical links. To estimate the parameters in GLMs, the iteratively reweighted least squares and the 
Newton-Raphson methods are the most common algorithms, which are used in most common statistical software packages such as SAS, S-Plus, and R.

Under certain conditions, MLEs of parameters in GLMs are asymptotically efficient and asymptotically normally distributed. That is,

$$
\hat{\boldsymbol{\beta}} \rightarrow N\left(\boldsymbol{\beta}, a(\phi)\left(\mathbf{X} W \mathbf{X}^{t}\right)^{-1}\right) .
$$

To make the statistical inference, we can consider asymptotic normality property for large sample size.

\subsection{Literature on Missing Data}

We often experience the missing data problem in practice, which could be either in responses or in covariates. The missing data may lead to invalid inference if missingness is not properly addressed. An appropriate approach is needed to deal with the missing data. A wide range of analysis of missing data mechanism with incomplete data has been done by several authors in the literature (e.g., Baker and Laird, 1988; Wu and Carroll, 1988; Brown, 1990; Ibrahim, 1990; Schluchter, 1992; Little, 1993, 1994; Diggle and Kenward, 1994; Vach, 1994; Robins, Rotnitzky and Zhao, 1995; Ibrahim, Lipsitz and Chen, 1999; Robins, Greenland and Hu, 1999).

Baker and Laird (1988) developed a log-linear model for categorical data when there are nonignorable missing data. They obtained maximum likelihood estimators by using the EM algorithm. They considered two different log-linear models to describe two associated regressions. First, they fitted a marginal model to make inferences about the regression of $Y$ on $X$, treating the nonresponse as a nuisance for the p-dimensional $X Y$ 
margin. Second, they used a nonresponse model to describe the regression of $R$ on $X$ and $Y$ for the full array assuming an interaction term of $Y R$ in the model.

Greenlees, Reece, and Zieschang (1982) proposed a parametric model using survey data for maximum likelihood estimators for both missing data mechanism and datagenerating process to deal with nonignorable nonresponse.

Later on, Tang, Little, and Raghunathan (2003) suggested a pseudo-likelihood for nonignorable nonresponse, implementing a parametric model on the data generating process. Their work was slightly different from Greenlees, Reece, and Zieschang (1982) in the sense that the missing data mechanism needed to be unspecified. This approach estimates population parameters in the complete data case considering the missing data mechanism as a nuisance parameter.

\subsection{Literature on Missing Covariates}

There is extensive research done with missing covariates by many authors in the biological research area. Little (1992) defined four different types of missing covariates patterns. First, univariate missing data where only one covariate values are missing. Second, monotone or nested missing data. Third, a special pattern where two covariates cannot be observed together and fourth, a general pattern of missing data without any special structure.

He provided six methods of estimating parameters for the regression models which are different in assumptions made about the mechanism to deal with missing values. He discussed these statistical methods to make a comparative study with missing covari- 
ates. He suggested three methods among six including the maximum likelihood method, Bayesian method and multiple imputation, which would be the better choice for dealing with missing covariate problems. However, for the large sample case, he preferred the maximum likelihood method, and small sample case, the Bayesian method or multiple imputation.

The generalized linear model is proposed by Ibrahim, Lipsitz and Chen (1999) for missing covariates with nonignorable missing data mechanism. They developed E step and $\mathrm{M}$ step of the EM algorithm for the covariates allowing them either to be categorical or continuous, where a Monte Carlo form of the EM algorithm is suggested through Gibbs sampler method for discrete covariates. Furthermore, they proposed a multinomial model that can be written as a logistic regression model or a sequence of one-dimensional conditional distributions with the missing data mechanism that may reduce the number of nuisance parameters. In Ibrahim, Chen, Lipsitz and Hemng (2005) a comparison of different methods is studied to make statistical inference in generalized linear models with missing covariates.

Yang, Belin and Boscardin (2005) proposed two alternative procedures to handle missing covariates and address the selection problem of a model in linear regression based on the Bayesian framework. One approach is called "impute then select" that produces multiplied imputed data sets first and then apply Bayesian variable selection to each of them subsequently. Another one is called "simultaneously impute and select", which inserts the steps for imputation and single Bayesian variable selection combining Gibbs sampling process. They used multivariate normal imputation models for incom- 
plete data by implementing conditional distribution in linear regression.

$\mathrm{Wu}$ and $\mathrm{Wu}$ (2001) developed a three-step multiple imputation method to estimate the parameters in nonlinear mixed effect models in the presence of missing covariates (MAR) by implementing the Gibbs sampler method. First, they fitted a non-linear mixed-effects models without covariates. Then they developed a multivariate linear model assigning missing covariates implemented by the Gibbs sampler. Finally, to analyze each dataset, they used the complete data method.

Sinha (2008) considered analyzing some interesting clinical data obtained from the Mental Health Program at St. Boniface Hospital Research Centre in Winnipeg, Manitoba, Canada. The study involved a group of 102 patients between the ages of 40 and 89 who underwent elective surgeries to repair an aortic medical condition. The patients were recruited prospectively from consecutive cases seen in the St. Boniface Outpatient Clinic for elective management of abdominal aortic aneurysm (AAA) during the period December 2000 to December 2003. A goal of the study was to observe occurrences of delirium in the AAA patients and to investigate factors that are predictive of the delirium experienced by those patients. The covariates considered in the analysis include "age", "packyrs" (number of packs of cigarettes smoked per day multiplied by the number of years the patient smoked), "etoh" (number of alcoholic drinks consumed in one year), "ppsychom" (number of psychometric medications taken prior to operation), and "pvasoact" (number of vasoactive medications taken prior to operation). Among these covariates, packyrs contained a large proportion of missing values, where the missingness was considered nonigrobale. The author proposed an efficient robust method in 
the framework of the maximum likelihood estimation by incorporating a missing data model into the observed data likelihood function. The method was shown to be robust against potential outliers in the data. 


\section{Chapter 3}

\section{Maximum Likelihood Estimation under Nonignorible Missing Covariates}

\subsection{Introduction}

In this chapter, we discuss the maximum likelihood method for estimating parameters in GLMs involving nonignorable missing covariates. We assume a binomial response model for the missing data mechanism. In the next section, we introduce the model and notation, and describe the likelihood method for analyzing GLMs with nonignorable missing covariates.

\subsection{Model and Notation}

\subsubsection{Full Model}

Let $\left\{\left(y_{i}, \mathbf{x}_{i}\right): i=1, \ldots, n\right\}$ be independent observations, where $y_{i}$ is the $i$ th response variables and $\mathbf{x}_{i}$ is the $i$ th random vector of $p$ covariates. Conditional on $\mathbf{x}_{i}$, assume 
that $y_{i}$ follows a distribution in the exponential family:

$$
f_{y_{i} \mid x_{i}}\left(y_{i} \mid \mathbf{x}_{i}, \boldsymbol{\beta}, \phi\right)=\exp \left[\left\{y_{i} \theta_{i}-b\left(\theta_{i}\right)\right\} / a(\phi)+c\left(y_{i}, \phi\right)\right]
$$

for some functions $a, b$ and $c$, and $\phi$ is a scaler dispersion parameter (McCullagh and Nelder, 1997). The canonical parameter is $\theta_{i}=\mathbf{x}_{i}^{t} \boldsymbol{\beta}$, where $\mathbf{x}_{i}$ is the $i$ th row of the design matrix $\mathbf{X}$, which may contain constant 1 to incorporate an intercept term.

The log-likelihood for Eq.(3.1) is obtained as

$$
l(\boldsymbol{\beta}, \phi \mid y, \mathbf{X})=\sum_{i=1}^{n}\left[\left\{y_{i} \theta_{i}-b\left(\theta_{i}\right)\right\} / a(\phi)+c\left(y_{i}, \phi\right)\right] .
$$

Here the dispersion parameter $\phi$ is fixed at unity, for simplicity. The ML estimating equation for $\boldsymbol{\beta}$ can be obtained by taking the derivative of Eq.(3.2) with respect to $\boldsymbol{\beta}$,

$$
\begin{aligned}
\frac{\partial l}{\partial \boldsymbol{\beta}}= & \sum_{i=1}^{n} \frac{\partial}{\partial \boldsymbol{\beta}}\left[y_{i}\left(\mathbf{x}^{T} \boldsymbol{\beta}\right)-b\left(\theta_{i}\right)\right] \\
& =\sum_{i=1}^{n}\left[y_{i} \mathbf{x}_{i}-\frac{\partial}{\partial \boldsymbol{\beta}}\left\{b\left(\theta_{i}\right)\right\}\right] \\
& =\sum_{i=1}^{n}\left[y_{i} \mathbf{x}_{i}-b^{\prime}\left(\theta_{i}\right) \mathbf{x}_{i}\right] \\
& =\sum_{i=1}^{n}\left[y_{i}-\mu_{i}\left(\boldsymbol{\beta}, \mathbf{x}_{i}\right)\right] \mathbf{x}_{i}=\mathbf{0}
\end{aligned}
$$

where $\mu_{i}\left(\boldsymbol{\beta}, \mathbf{x}_{i}\right)=E\left(y_{i} \mid \mathbf{x}_{i}, \boldsymbol{\beta}\right)=b^{\prime}\left(\theta_{i}\right)$.

\subsubsection{Model for Covariates}

Specification of a parametric model plays an important role for the missing covariates (Ibrahim, Chen, Lipsitz and Herring, 1999). In a specified parametric model for the covariates, the indexing parameters of this distribution are usually observed as nuisance 
parameters, which are not parameters of inferential interest. To reduce the number of nuisance parameters, some strategies are needed to be implemented when the covariate distribution is specified since the parameter estimation becomes computationally inefficient and intensive due to a large number of nuisance parameters.

When the missing covariates are categorical, Lipsitz and Ibrahim (1996) proposed a joint distribution of the covariates as a product of one-dimensional conditional distribution to reduce the number of nuisance parameters in the covariate distribution.

Here, we consider a joint distribution of the $p$-dimentional covariate vector $\mathbf{x}_{i}=\left(x_{1 i}, x_{2 i}, \ldots, x_{p i}\right)^{t}$, denoted by $f\left(\mathbf{x}_{i} \mid \boldsymbol{\alpha}\right)$, as used in Ibrahim, Lipsitz and Chen (1999). We can write

$$
\begin{array}{r}
f\left(x_{1 i}, \ldots x_{p i} \mid \boldsymbol{\alpha}\right)= \\
\times f\left(x_{p i} \mid x_{1 i}, \ldots x_{p-1, i}, \boldsymbol{\alpha}_{p-1, i}\right) \\
\ldots \\
\quad \times f\left(x_{1 i}, \ldots x_{p-2, i}, x_{1 i}, \boldsymbol{\alpha}_{p-1}\right) \\
\ldots\left(x_{1 i} \mid \boldsymbol{\alpha}_{1}\right),
\end{array}
$$

where $\boldsymbol{\alpha}_{j}$ is a vector of parameters for the $j$ th conditional distribution that are distinct and $\boldsymbol{\alpha}=\left(\boldsymbol{\alpha}_{1}, \ldots, \boldsymbol{\alpha}_{p}\right)$. The above model is quite useful for categorical covariates as well as continuous covariates when there is no natural joint distribution to specify. Eq.(3.4) indicates a sequence of one-dimensional conditional distributions for $x_{i}$. When the covariates are completely observed, models are not necessarily to be specified and then the above equation is used for the missing covariates only. 
Eq.(3.4) is also useful for mixed covariate case, which often occurs in clinical trials (Ibrahim, Lipsitz and Chen, 1999). When the categorical and continuous covariates are both missing, specifying a joint distribution is quite unrealistic. In this situation, model (3.4) works well and reduce a large number of nuisance parameters. One approach for this mixed covariates case would be first specifying a one-dimensional distribution for the continuous covariates and then attaining the one-dimensional distribution for the categorical covariates by conditioning on the continuous covariates. Since each of the one dimensional conditional distributions in Eq.(3.4) has a distribution of the exponential family, the property of log-concavity still exists.

\subsubsection{Model for Missing Data}

Models are required when the missing data mechanism is nonignorable. Similarly to Ibrahim, Lipsitz and Chen (1999), here we consider a joint log-linear model for missing data mechanism $f\left(\mathbf{v}_{i} \mid y_{i}, \mathbf{x}_{i}, \boldsymbol{\tau}\right)$ as the product of a sequence of one-dimensional conditional distributions. It can be written as

$$
\begin{array}{r}
f\left(v_{1 i}, \ldots v_{p i} \mid y_{i}, \mathbf{x}_{i}, \boldsymbol{\tau}\right)=f\left(v_{p i} \mid v_{1 i}, \ldots v_{p-1}, i\right. \\
\end{array} \begin{array}{r}
\left.y_{i}, \mathbf{x}_{i}, \boldsymbol{\tau}_{p}\right) \\
\times f\left(v_{p-1, i} \mid v_{1 i}, \ldots v_{p-2, i}, y_{i}, \mathbf{x}_{i}, \boldsymbol{\tau}_{p-1}\right) \\
\ldots \\
\quad \times f\left(v_{2 i} \mid v_{1 i}, y_{i}, \mathbf{x}_{i}, \boldsymbol{\tau}_{2}\right) f\left(v_{1 i} \mid y_{i}, \mathbf{x}_{i}, \boldsymbol{\tau}_{1}\right),
\end{array}
$$

where the $j$ th element of $\boldsymbol{\tau}_{j}$ in $\boldsymbol{\tau}=\left(\boldsymbol{\tau}_{1}, \ldots \boldsymbol{\tau}_{p}\right)$ represents a vector of parameters, and

$v_{j i}(j=1,2, \ldots, p)$ is a binary variable indicating the missingness of the $j$ th covariate $x_{j i}$. 
For the missing data model (3.5), the likelihood score equations for estimating the nuisance parameters are obtained as

$$
\sum_{i=1}^{n} E\left[\frac{\partial \log f\left(v_{1 i}, \ldots v_{p i} \mid y_{i}, \mathbf{x}_{i}, \boldsymbol{\tau}\right)}{\partial \boldsymbol{\tau}} \mid y_{i}, \mathbf{x}_{i}, \boldsymbol{\tau}\right]=\mathbf{0}
$$

Assuming a logistic model for each of the conditional distribution in (3.5), we obtain the estimating equations for $\boldsymbol{\tau}_{p}$ as

$$
\sum_{i=1}^{n} E\left[\left\{v_{p i}-\eta_{p i}\left(\boldsymbol{\tau}_{p}, \mathbf{x}_{i}{ }^{*}\right)\right\} \mathbf{x}_{i}{ }^{*} \mid y_{i}, \mathbf{x}_{\mathrm{obs}, i}, \mathbf{v}_{i}\right]=\mathbf{0}
$$

where $\mathbf{x}_{i}{ }^{*}=\left(v_{1 i}, \ldots v_{p-1}, i, y_{i}, \mathbf{x}_{i}\right)$ and $\eta_{p i}\left(\boldsymbol{\tau}_{p}, \mathbf{x}_{i}{ }^{*}\right)$ is the expected value of $v_{p i}$ when $\mathbf{x}_{i}{ }^{*}$ is given.

When the missingness of one variable affects the probability of missingness in others, the modelling approach (3.5) provides a flexible specification of the joint distribution of the missing-data indicators. It also enables the random sampling to approximate the conditional expectation for the score equation which is obtained from the conditional distribution of the missing covariates given the observed data (Sinha, 2008). The density functions of the right-hand side of Eq.(3.5) satisfy the log-concavity property since each of the univariate distribution can be expressed as a logistic regression. This property facilitates the computations of the maximum likelihood estimates.

In missing data problems, selection of appropriate covariates for the missing data 
mechanism is a crucial issue. To check the adequacy of fits of various models in the presence of missing data, we can use the likelihood ratio or Akaike information criterion. Ibrahim, Lipsitz and Chen (1999) suggested that one should not use a very large model for the missing data mechanism, since the model can easily become unidentifiable due to overparameterization. Baker and Laird (1988) mentioned that when the missing data mechanism is nonignorable, the issue of estimability can often arise and characterization of the set of estimable parameters for a certain class of models is still under investigation.

\subsection{Methods of Estimation}

\subsubsection{Estimation under Missing Covariates}

Suppose $\left\{\left(y_{i}, \mathbf{x}_{i}\right): i=1, \ldots, n\right\}$ is a set of data that might occur when there is no missing value. Also, consider $\mathbf{x}_{\mathrm{obs}, i}$ is the observed values and $\mathbf{x}_{\mathrm{mis}, i}$ the missing values of $\mathbf{x}_{i}$. Assuming a nonmonotonic pattern of missing data in $\mathbf{x}_{i}$, some permutation can be written as $\mathbf{x}_{i}=\left(\mathbf{x}_{\mathrm{obs}, i}, \mathbf{x}_{\mathrm{mis}, i}\right)$, where $\mathbf{x}_{\mathrm{mis}, i}$ is the $p_{i} \times 1$ vector of missing values of $\mathbf{x}_{i}$.

The joint density function of the observed data may be obtained as

$$
f\left(y_{i}, \mathbf{x}_{\mathrm{obs}, i}\right)=\int f\left(y_{i}, \mathbf{x}_{\mathrm{obs}, i}, \mathbf{x}_{\mathrm{mis}, i},\right) d \mathbf{x}_{\mathrm{mis}, i}
$$

When the missing data mechanism is nonignorible, it is important to incorporate the missing data model into the observed data likelihood function.

Define 


$$
v_{i j}=\left\{\begin{array}{lll}
1 & \text { if } & x_{i j} \text { missing } \\
0 & \text { if } & x_{i j} \text { observed }
\end{array}\right.
$$

where $x_{i j}$ is the $j$ th covariate in $\mathbf{x}_{i} ;(j=1, \ldots p)$ and $\mathbf{v}_{i}=\left(v_{i 1}, v_{i 2}, \ldots v_{i p}\right)^{t}$ is the vector of missingness indicators.

The conditional distribution of $\mathbf{v}_{i}$ has a multinominal distribution $f\left(\mathbf{v}_{i} \mid y_{i}, \mathbf{x}_{i}, \boldsymbol{\tau}\right)$ that depends on some parameters $\boldsymbol{\tau}$. Assume that the random vector $\mathbf{x}_{i}$ follows a density $f\left(\mathbf{x}_{i} \mid \boldsymbol{\alpha}\right)$ that depends on some parameter $\boldsymbol{\alpha}$. Now, for the $i$ th observation, we have the actual observed data that has the joint density $f\left(y_{i}, \mathbf{x}_{\mathrm{obs}, i}, \mathbf{v}_{i}\right)$ which can be obtained by integrating out $f\left(y_{i}, \mathbf{x}_{i}, \mathbf{v}_{i}\right)$ with respect to $\mathbf{x}_{\mathrm{mis}, i}$. It can be written as

$$
\begin{array}{r}
f\left(y_{i}, \mathbf{x}_{\mathrm{obs}, i}, \mathbf{v}_{i} \mid \boldsymbol{\beta}, \boldsymbol{\alpha}, \boldsymbol{\tau}\right)=\int f\left(y_{i} \mid \mathbf{x}_{\mathrm{obs}, i}, \mathbf{x}_{\mathrm{mis}, i}, \boldsymbol{\beta}\right) f\left(\mathbf{x}_{\mathrm{obs}, i}, \mathbf{x}_{\mathrm{mis}, i}, \mid \boldsymbol{\alpha}\right) \\
\times f\left(\mathbf{v}_{i} \mid y_{i}, \mathbf{x}_{\mathrm{obs}, i}, \mathbf{x}_{\mathrm{mis}, i}, \boldsymbol{\tau}\right) d \mathbf{x}_{\mathrm{mis}, i}
\end{array}
$$

When the missing data mechanism is nonignorable, the inferences on $\boldsymbol{\beta}$ should be based on the full likelihood. This likelihood cannot normally be evaluated in a closed form since the $i$ th unit involves an integral with dimension equal to the dimension of missing observation $\mathbf{x}_{\text {mis }, i}$ when calculating the density $f\left(y_{i}, \mathbf{x}_{\mathrm{obs}, i}, \mathbf{v}_{i} \mid \boldsymbol{\beta}, \boldsymbol{\alpha}, \boldsymbol{\tau}\right)$ for the actual observed data. The objective of the study is to review the algorithms for calculating the ML estimates under the full likelihood. We can write the full likelihood as

$$
\begin{array}{r}
L_{i}=f\left(y_{i}, \mathbf{x}_{\mathrm{obs}, i}, \mathbf{v}_{i}\right) \\
=\int f\left(y_{i} \mid \mathbf{x}_{\mathrm{obs}, i}, \mathbf{x}_{\mathrm{mis}, i}, \mathbf{v}_{i}\right) d \mathbf{x}_{\mathrm{mis}, i}
\end{array}
$$


The log-likelihood can be written as

$$
l_{i}=\log L_{i}=\log f\left(y_{i}, \mathbf{x}_{\mathrm{obs}, i}, \mathbf{v}_{i}\right)
$$

Suppose that $\boldsymbol{\gamma}=(\boldsymbol{\beta}, \boldsymbol{\alpha}, \boldsymbol{\tau})$. The score equations can be obtained by taking derivatives of the log-likelihood with respect to $\gamma$. We can write

$$
\begin{aligned}
\frac{\partial l i}{\partial \boldsymbol{\gamma}}= & \frac{1}{f\left(y_{i}, \mathbf{x}_{\mathrm{obs}, i}, \mathbf{v}_{i}\right)} \frac{\partial}{\partial \gamma} f\left(y_{i}, \mathbf{x}_{\mathrm{obs}, i}, \mathbf{v}_{i}\right) \\
& =\frac{1}{f\left(y_{i}, \mathbf{x}_{\mathrm{obs}, i}, \mathbf{v}_{i}\right)} \frac{\partial}{\partial \boldsymbol{\gamma}} \int f\left(y_{i}, \mathbf{x}_{i}, \mathbf{v}_{i}\right) d \mathbf{x}_{\mathrm{mis}, i} \\
& =\frac{1}{f\left(y_{i}, \mathbf{x}_{\mathrm{obs}, i}, \mathbf{v}_{i}\right)} \int \frac{\partial \log f\left(y_{i}, \mathbf{x}_{i}, \mathbf{v}_{i}\right)}{\partial \boldsymbol{\gamma}} f\left(y_{i}, \mathbf{x}_{i}, \mathbf{v}_{i}\right) d \mathbf{x}_{\mathrm{mis}, i} \\
& =\int \frac{\partial \log f\left(y_{i}, \mathbf{x}_{i}, \mathbf{v}_{i}\right)}{\partial \boldsymbol{\gamma}} \frac{f\left(y_{i}, \mathbf{x}_{i}, \mathbf{v}_{i}\right)}{f\left(y_{i}, \mathbf{x}_{\mathrm{obs}, i}, \mathbf{v}_{i}\right)} d \mathbf{x}_{\mathrm{mis}, i} \\
& =\int \frac{\partial \log f\left(y_{i}, \mathbf{x}_{i}, \mathbf{v}_{i}\right)}{\partial \boldsymbol{\gamma}} f\left(\mathbf{x}_{\mathrm{mis}, i} \mid y_{i}, \mathbf{x}_{\mathrm{obs}, i}, \mathbf{v}_{i}\right) d \mathbf{x}_{\mathrm{mis}, i} \\
& =E\left[\frac{\partial \log f\left(y_{i}, \mathbf{x}_{i}, \mathbf{v}_{i}\right)}{\partial \boldsymbol{\gamma}} \mid y_{i}, \mathbf{x}_{\mathrm{obs}, i}, \mathbf{v}_{i}\right] \\
& =E\left[\frac{\partial}{\partial \boldsymbol{\gamma}} \log \left\{f\left(y_{i} \mid \mathbf{x}_{i}\right) f\left(\mathbf{x}_{i}\right) f\left(\mathbf{v}_{i} \mid y_{i}, \mathbf{x}_{i}\right)\right\} \mid y_{i}, \mathbf{x}_{\mathrm{obs}, i}, \mathbf{v}_{i}\right] \\
& =E\left[\frac{\partial}{\partial \boldsymbol{\gamma}}\left\{\log f\left(y_{i} \mid \mathbf{x}_{i}\right)+\log f\left(\mathbf{x}_{i}\right)+\log f\left(\mathbf{v}_{i} \mid y_{i}, \mathbf{x}_{i}\right)\right\} \mid y_{i}, \mathbf{x}_{\mathrm{obs}, i}, \mathbf{v}_{i}\right]
\end{aligned}
$$

When the observed data $\left(y_{i}, \mathbf{x}_{\mathrm{obs}, i}, \mathbf{v}_{i}\right)$ are given, the conditional expectations are taken with respect to $\mathbf{x}_{\mathrm{mis}, i}$. So we can find the score equations as 


$$
\begin{aligned}
E\left[\frac{\partial \log f\left(y_{i} \mid \mathbf{x}_{i}\right)}{\partial \boldsymbol{\beta}} \mid y_{i}, \mathbf{x}_{\mathrm{obs}, i}, \mathbf{v}_{i}\right] & =\mathbf{0}, \\
E\left[\frac{\partial \log f\left(\mathbf{x}_{i}\right)}{\partial \boldsymbol{\alpha}} \mid y_{i}, \mathbf{x}_{\mathrm{obs}, i}, \mathbf{v}_{i}\right] & =\mathbf{0}, \\
E\left[\frac{\partial \log f\left(v_{i} \mid y_{i}, \mathbf{x}_{i}\right)}{\partial \boldsymbol{\tau}} \mid y_{i}, \mathbf{x}_{\mathrm{obs}, i}, \mathbf{v}_{i}\right] & =\mathbf{0} .
\end{aligned}
$$

Our primary interest lies in the estimation of $\boldsymbol{\beta}$ while $\boldsymbol{\alpha}$ and $\boldsymbol{\tau}$ being viewed as nuisance parameters. From the above equations, Eq.(3.10) can be solved by using Newton-Raphson or scoring approach as used for complete data in generalized linear models. For the exponential family (3.1), Eq.(3.10) for the ML estimates of $\boldsymbol{\beta}$ takes the following form

$$
\sum_{i=1}^{n} E\left[\left\{y_{i}-\mu_{i}\left(\boldsymbol{\beta}, \mathbf{x}_{i}\right)\right\} \mathbf{x}_{i} \mid y_{i}, \mathbf{x}_{\mathrm{obs}, i}, \mathbf{v}_{i}\right]=\mathbf{0}
$$

where $\mathbf{x}_{i}=\left(\mathbf{x}_{\mathrm{obs}, i}, \mathbf{x}_{\mathrm{m} i s, i}\right)$. This score function is proportional to the observed values of $y$ and $x$. For the missing data mechanism, the ML estimate of $\boldsymbol{\tau}$ in Eq.(3.12) can be solved in a similar way. Since Eq.(3.11) involves only the distribution of $\mathbf{x}$, typically it is easy to solve for the ML estimates of $\boldsymbol{\alpha}$.

\subsubsection{Asymptotic Variance of the ML Estimator}

We can show that 


$$
\begin{aligned}
E\left(y_{i} \mid \mathbf{x}_{i}, \boldsymbol{\beta}\right)= & E\left(\frac{\partial l}{\partial \theta_{i}}\right) \\
& =\frac{\partial b\left(\theta_{i}\right)}{\partial \theta_{i}} \\
& =b^{\prime}\left(\theta_{i}\right) \\
& =\mu_{i}\left(\boldsymbol{\beta}, \mathbf{x}_{i}\right),
\end{aligned}
$$

where $\theta_{i}=\mathbf{x}_{i}^{t} \boldsymbol{\beta}$. The asymptotic variance of the ML estimator of $\boldsymbol{\beta}$ can be obtained from Eq.(3.10), using the Fisher information which takes the form

$$
\begin{aligned}
\mathbf{I}_{0}(\boldsymbol{\beta})= & -\sum_{i=1}^{n} E\left\{\partial \mathbf{U}(\boldsymbol{\beta}) / \partial \boldsymbol{\beta}^{T} \mid y_{i}, \mathbf{x}_{\mathrm{obs}, i}, \mathbf{v}_{i}\right\}-\sum_{i=1}^{n} E\left\{\mathbf{U}(\boldsymbol{\beta}) \mathbf{U}(\boldsymbol{\beta})^{T} \mid y_{i}, \mathbf{x}_{\mathrm{obs}, i}, \mathbf{v}_{i}\right\} \\
& +\sum_{i=1}^{n} E\left\{\mathbf{U}(\boldsymbol{\beta}) \mid y_{i}, \mathbf{x}_{\mathrm{obs}, i}, \mathbf{v}_{i}\right\} E\left\{\mathbf{U}(\boldsymbol{\beta})^{T} \mid y_{i}, \mathbf{x}_{\mathrm{obs}, i}, \mathbf{v}_{i}\right\}
\end{aligned}
$$

where $\mathbf{U}(\boldsymbol{\beta})=\partial \log f\left(y_{i} \mid \mathbf{x}_{i}, \boldsymbol{\beta}\right) / \partial \boldsymbol{\beta}$.

\subsubsection{Newton-Raphson Method}

Solving a system of nonlinear equations is not feasible as it can in the case of linear equations since the solution cannot be derived algebraically. The solution must be numerically estimated using an iterative process. The most popular method for solving these nonlinear equations is Newton-Raphson method.

The Newton-Raphson method is a powerful technique for solving nonlinear equations numerically. To obtain the maximum likelihood estimator of $\boldsymbol{\beta}$, here we use a Newton-Raphson algorithm to solve Eq.(3.13). This method is a linear Tylor series approximation where we expand the left side of Eq.(3.13) as a function of $\boldsymbol{\beta}$.

We can write the left side of Eq.(3.13) without the expectation in the form

$$
\sum_{i=1}^{n}\left[\left\{y_{i}-\mu_{i}\left(\boldsymbol{\beta}, \mathbf{x}_{i}\right)\right\} \mathbf{x}_{i}\right]
$$


Using first order Tylor series around some initial value $\boldsymbol{\beta}_{0}$ and also using the Fisher scoring technique, we can write

$$
\begin{aligned}
\sum_{i=1}^{n} & \left\{y_{i}-\mu_{i}\left(\boldsymbol{\beta}, \mathbf{x}_{i}\right)\right\} \mathbf{x}_{i} \\
& \cong \sum_{i=1}^{n}\left\{y_{i}-\mu_{i}\left(\boldsymbol{\beta}_{0}, \mathbf{x}_{i}\right)\right\} \mathbf{x}_{i} \\
& +\sum_{i=1}^{n}\left[\left.\left(\partial / \partial \theta_{i}\right)\left\{y_{i}-\mu_{i}\left(\boldsymbol{\beta}, \mathbf{x}_{i}\right)\right\}\right|_{\left.\boldsymbol{\beta}=\boldsymbol{\beta}_{0}\right]}\right. \\
& \times \mathbf{x}_{i} \mathbf{x}_{i}^{t}\left(\boldsymbol{\beta}-\boldsymbol{\beta}_{0}\right) \\
& =\sum_{i=1}^{n} d_{i}\left(\boldsymbol{\beta}_{0}, y_{i}, \mathbf{x}_{i}\right) \mathbf{x}_{i}-\sum_{i=1}^{n} d_{i}^{\prime}\left(\boldsymbol{\beta}_{0}, y_{i}, \mathbf{x}_{i}\right) \mathbf{x}_{i} \mathbf{x}_{i}^{t}\left(\boldsymbol{\beta}-\boldsymbol{\beta}_{0}\right)
\end{aligned}
$$

where $d_{i}\left(\boldsymbol{\beta}, y_{i}, \mathbf{x}_{i}\right)=y_{i}-\mu_{i}\left(\boldsymbol{\beta}, \mathbf{x}_{i}\right)$ and $d_{i}^{\prime}\left(\boldsymbol{\beta}_{0}, y_{i}, \mathbf{x}_{i}\right)=-\left(\partial / \partial \theta_{i}\right) d_{i}\left(\boldsymbol{\beta}, y_{i}, \mathbf{x}_{i}\right)$ evaluated at $\boldsymbol{\beta}_{0}$.

Approximation of Eq.(3.16) leads to an iterative equation for $\boldsymbol{\beta}$, in the form

$$
\begin{aligned}
\boldsymbol{\beta}^{(j+1)}= & \boldsymbol{\beta}^{(j)}+\left[\sum_{i=1}^{n} E\left\{d_{i}^{\prime}\left(\boldsymbol{\beta}^{(j)}, y_{i}, \mathbf{x}_{i}\right) \mathbf{x}_{i} \mathbf{x}_{i}^{t} \mid y_{i}, \mathbf{x}_{\mathrm{obs}, i}, \mathbf{v}_{i}\right\}\right]^{-1} \\
& \times \sum_{i=1}^{n} E\left\{d_{i}\left(\boldsymbol{\beta}^{(j)}, y_{i}, \mathbf{x}_{i}\right) \mathbf{x}_{i} \mid y_{i}, \mathbf{x}_{\mathrm{obs}, i}, \mathbf{v}_{i}\right\}
\end{aligned}
$$

To get the maximum likelihood estimates of $\boldsymbol{\beta}$, Eq.(3.17) requires the calculation of conditional expectations.

\subsubsection{Asymptotics}

Based on the maximum likelihood, asymptotic existence, consistency and asymptotic normality of fixed effect estimators are proved by Haberman (1977) and Fahrmeir and Kaufmann (1985) under suitable conditions. Some conditions are provided in Haberman (1977) for the asymptotic existence of the MLE by the use of the general theory 
for exponential models derived in Berk (1972) and Barndorff-Nielsen (1983).

Under suitable regularity conditions, $n^{1 / 2}(\hat{\boldsymbol{\beta}}-\boldsymbol{\beta})$ is asymptotically distributed as Gaussian with mean zero and covariance matrix consistently estimated by $\hat{\mathbf{C}}^{-1} \hat{\mathbf{B}} \hat{\mathbf{C}}^{-1}$, where

$$
\hat{\mathbf{B}}=n^{-1} \sum_{i=1}^{n} \Phi_{i}(\boldsymbol{\beta}) \Phi_{i}(\boldsymbol{\beta})^{t},
$$

with

$$
\begin{gathered}
\Phi_{i}(\boldsymbol{\beta})=E\left[\left\{y_{i}-\mu_{i}\left(\boldsymbol{\beta}, \mathbf{x}_{i}\right)\right\} \mathbf{x}_{i} \mid y_{i}, \mathbf{x}_{\mathrm{obs}, i}, \mathbf{v}_{i}, \boldsymbol{\gamma}\right] \\
=E\left[d_{i}\left(\boldsymbol{\beta}, \mathbf{x}_{i}\right) \mathbf{x}_{i} \mid y_{i}, \mathbf{x}_{\mathrm{obs}, i}, \mathbf{v}_{i}, \boldsymbol{\gamma}\right]
\end{gathered}
$$

and

$$
\begin{aligned}
\hat{\mathbf{C}}= & -n^{-1} \sum_{i=1}^{n} E\left[\left(\frac{\partial d_{i}\left(\boldsymbol{\beta}, \mathbf{x}_{i}\right)}{\partial \boldsymbol{\beta}}\right) \mathbf{x}_{i} \mid y_{i}, \mathbf{x}_{\mathrm{obs}, i}, \mathbf{v}_{i}, \boldsymbol{\gamma}\right] \\
& -n^{-1} \sum_{i=1}^{n} E\left[d_{i}\left(\boldsymbol{\beta}, \mathbf{x}_{i}\right) \mathbf{x}_{i}\left(\frac{\partial \log f\left(y_{i} \mid \mathbf{x}_{i}\right)}{\partial \boldsymbol{\beta}}\right) \mid y_{i}, \mathbf{x}_{\mathrm{obs}, i}, \mathbf{v}_{i}, \boldsymbol{\gamma}\right] \\
& +n^{-1} \sum_{i=1}^{n} E\left[d_{i}\left(\boldsymbol{\beta}, \mathbf{x}_{i}\right) \mathbf{x}_{i} \mid y_{i}, \mathbf{x}_{\mathrm{obs}, i}, \mathbf{v}_{i}, \boldsymbol{\gamma}\right] \\
& \times E\left[\left(\frac{\partial \log f\left(y_{i} \mid \mathbf{x}_{i}\right)}{\partial \boldsymbol{\beta}}\right) \mid y_{i}, \mathbf{x}_{\mathrm{obs}, i}, \mathbf{v}_{i}, \boldsymbol{\gamma}\right]
\end{aligned}
$$

where $d_{i}\left(\boldsymbol{\beta}, \mathbf{x}_{i}\right)=y_{i}-\mu_{i}\left(\boldsymbol{\beta}, \mathbf{x}_{i}\right)$.

Under regularity conditions and using a Taylor series expansion, $n^{1 / 2}(\hat{\boldsymbol{\beta}}-\boldsymbol{\beta})$ can be approximated from Eq.(3.13) by

$$
\left[-n^{-1} \partial \boldsymbol{\Phi}(\boldsymbol{\beta}) / \partial \boldsymbol{\beta}\right]^{-1}\left[n^{-1 / 2} \boldsymbol{\Phi}(\boldsymbol{\beta})\right]
$$


where $\boldsymbol{\Phi}(\boldsymbol{\beta})=\sum_{i=1}^{n} \Phi_{i}(\boldsymbol{\beta})$.

In Eq.(3.18), the function $n^{-1 / 2} \boldsymbol{\Phi}(\boldsymbol{\beta})$ generally has an asymptotic normal distribution as $n \rightarrow \infty$ with mean zero and covariance matrix

$$
\mathbf{B}=\lim _{n \rightarrow \infty} n^{-1} \sum_{i=1}^{n} \operatorname{var}\left\{\Phi_{i}(\boldsymbol{\beta})\right\}=\lim _{n \rightarrow \infty} n^{-1} \sum_{i=1}^{n} E\left\{\Phi_{i}(\boldsymbol{\beta}) \Phi_{i}(\boldsymbol{\beta})^{t}\right\},
$$

where the expectation is with respect to the marginal distribution of $y_{i}$.

Now we can show that $-n^{-1} \partial \Phi(\boldsymbol{\beta}) / \partial \boldsymbol{\beta}$ converges in probability to its asymptotic mean when $n \rightarrow \infty$. We have

$$
\begin{aligned}
& n^{-1} \partial \boldsymbol{\Phi}(\boldsymbol{\beta}) / \partial \boldsymbol{\beta} \\
& \quad=n^{-1} \sum_{i=1}^{n} E\left[\left(\frac{\partial d_{i}\left(\boldsymbol{\beta}, \mathbf{x}_{i}\right)}{\partial \boldsymbol{\beta}}\right) \mathbf{x}_{i} \mid y_{i}, \mathbf{x}_{\mathrm{obs}, i}, \mathbf{v}_{i}, \boldsymbol{\gamma}\right] \\
& \quad+n^{-1} \sum_{i=1}^{n} E\left[d_{i}\left(\boldsymbol{\beta}, \mathbf{x}_{i}\right) \mathbf{x}_{i}\left(\frac{\partial \log f\left(y_{i} \mid \mathbf{x}_{i}\right)}{\partial \boldsymbol{\beta}}\right) \mid y_{i}, \mathbf{x}_{\mathrm{obs}, i}, \mathbf{v}_{i}, \boldsymbol{\gamma}\right] \\
& \quad-n^{-1} \sum_{i=1}^{n} E\left[d_{i}\left(\boldsymbol{\beta}, \mathbf{x}_{i}\right) \mathbf{x}_{i} \mid y_{i}, \mathbf{x}_{\mathrm{obs}, i}, \mathbf{v}_{i}, \boldsymbol{\gamma}\right] \\
& \quad \times E\left[\left(\frac{\partial \log f\left(y_{i} \mid \mathbf{x}_{i}\right)}{\partial \boldsymbol{\beta}}\right) \mid y_{i}, \mathbf{x}_{\mathrm{obs}, i}, \mathbf{v}_{i}, \boldsymbol{\gamma}\right]
\end{aligned}
$$

where $d_{i}\left(\boldsymbol{\beta}, \mathbf{x}_{i}\right)=y_{i}-\mu_{i}\left(\boldsymbol{\beta}, \mathbf{x}_{i}\right)$. The matrix $-n^{-1} \partial \Phi(\boldsymbol{\beta}) / \partial \boldsymbol{\beta}$ converges in probability as $n \rightarrow \infty$ to the matrix

$$
\mathbf{C}=\lim _{n \rightarrow \infty} n^{-1} \sum_{i=1}^{n} E\left\{\dot{\Phi}_{i}(\boldsymbol{\beta})\right\}
$$


where

$$
\begin{aligned}
\dot{\Phi}_{i}(\boldsymbol{\beta})= & E\left[\left(\frac{\partial d_{i}\left(\boldsymbol{\beta}, \mathbf{x}_{i}\right)}{\partial \boldsymbol{\beta}}\right) \mathbf{x}_{i} \mid y_{i}, \mathbf{x}_{\mathrm{obs}, i}, \mathbf{v}_{i}, \boldsymbol{\gamma}\right] \\
& +E\left[d_{i}\left(\boldsymbol{\beta}, \mathbf{x}_{i}\right) \mathbf{x}_{i}\left(\frac{\partial \log f\left(y_{i} \mid \mathbf{x}_{i}\right)}{\partial \boldsymbol{\beta}}\right) \mid y_{i}, \mathbf{x}_{\mathrm{obs}, i}, \mathbf{v}_{i}, \boldsymbol{\gamma}\right] \\
& -E\left[d_{i}\left(\boldsymbol{\beta}, \mathbf{x}_{i}\right) \mathbf{x}_{i} \mid y_{i}, \mathbf{x}_{\mathrm{obs}, i}, \mathbf{v}_{i}, \boldsymbol{\gamma}\right] \\
& \times E\left[\left(\frac{\partial \log f\left(y_{i} \mid \mathbf{x}_{i}\right)}{\partial \boldsymbol{\beta}}\right) \mid y_{i}, \mathbf{x}_{\mathrm{obs}, i}, \mathbf{v}_{i}, \boldsymbol{\gamma}\right]
\end{aligned}
$$

By combining (3.19) and $(3.20), n^{1 / 2}(\hat{\boldsymbol{\beta}}-\boldsymbol{\beta})$ is asymptotically normally distributed with mean zero and covariance matrix $\mathbf{C}^{-1} \mathbf{B} \mathbf{C}^{-1}$. This covariance matrix is estimated by $\hat{\mathbf{C}}^{-1} \hat{\mathbf{B}} \hat{\mathbf{C}}^{-1}$, where $\hat{\mathbf{B}}$ and $\hat{\mathbf{C}}$ were defined earlier, with $\boldsymbol{\beta}$ being replaced by its ML estimator $\hat{\boldsymbol{\beta}}$. 


\section{Chapter 4}

\section{Binary Regression}

\subsection{Introduction}

A binomial logistic regression is often referred to simply as logistic regression. The logistic regression is a powerful and widely used statistical way of modelling a binary outcome which takes the value "0" or "1" like success or failure with one or more explanatory variables.

The logistic regression is used to predict an outcome variable that is categorical from predictor variables which may be continuous or categorical. Since categorical outcome variable violates the assumption of linearity in normal regression, one can consider the logistic regression rather than the linear regression. Also, when the outcome variable is dichotomous and the predictors tend to show a linear relationship, the logistic regression is used. Suppose that we are interested in predicting whether a patient has a given disease (e.g., diabetes, coronary heart disease), based on observed characteristics of the patient such as age, sex, body mass index, results of various blood tests, etc.. The outcome (response) variable is binary $(0 / 1)$ and the predictor variables of interest are 
several observed characteristics of the patient. In this case, the logistic regression can be used for modelling the binary response as a function of the predictors (Freedman, D. A., 2009).

The logistic regression is frequently used in social and medical sciences, as the outcomes from these experiments are usually binary. When the outcome variable is not discrete, the logistic regression is used by taking a logarithmic transformation on the outcome variable, which allows us to model a nonlinear association in a linear way. It shows the linear regression equation in logarithmic terms which is called the logit. When there is more than one risk factors and the odds ratio needs to be adjusted, it is better to use the logistic regression.

In this chapter, we introduce the response model and notation to define the generalized linear models for binary data and describe the maximum likelihood method for estimating parameters in the binary logistic model. We also present results from a simulation study, which was carried out to investigate the empirical properties of the maximum likelihood approach.

\subsection{Model and Notation}

\subsubsection{Binary Logistic Model}

In generalized linear models (GLMs), the logistic regression analysis is often used to investigate the relationship between a binary response variable and a set of explanatory variables. A binary response consists, for example, of success or failure. In the case of disease studies, the outcome is denoted as $Y_{1}=1$ if the disease is present or $Y_{2}=0$, 
otherwise. To analyze this kind of variable which does not follow a normal distribution, usually a link function (LOGIT) is used.

Let $y_{i}$ be the binary response variable that contains only two values, " 0 " and " 1 ". Then we can define it as

$$
y_{i}=\left\{\begin{array}{cc}
1 & \text { if success } \\
0 & \text { if failure }
\end{array}\right.
$$

We treat $y_{i}$ as a realization of a random variable $Y_{i}$, which takes the values one and zero with probabilities $p_{i}$ and $1-p_{i}$, respectively. Here, $p_{i}$ indicates the probability of success and $1-p_{i}$ indicates the probability of failure. The distribution of $Y_{i}$ is called a Bernoulli distribution with parameter $p_{i}$, and can be written as

$$
\operatorname{Pr}\left(Y_{i}=y_{i}\right)=p_{i}^{y_{i}}\left(1-p_{i}\right)^{1-y_{i}}
$$

where $y_{i}=0,1$. The expected value and variance of $Y_{i}$ can be obtained in the form

$$
E\left(Y_{i}\right)=\mu_{i}=p_{i}
$$

and

$$
\operatorname{var}\left(Y_{i}\right)={\sigma_{i}^{2}}^{2}=p_{i}\left(1-p_{i}\right)
$$

where the mean and variance depend on the underlying parameter $p_{i}$.

Again, suppose that $y_{i}$ can be treated as a realization of $Y_{i}$ which takes values from 1 to $n_{i}$. Assume that $p_{i}$ is the probability of success in a given trial, $n_{i}$ is the number of $i$ th independent observations and $y_{i}$ is the number of units that succeed. Then, the 
binomial distribution of $Y_{i}$ with parameters $n_{i}$ and $p_{i}$ can be written as

$$
Y_{i} \sim B\left(n_{i}, p_{i}\right)
$$

The general form of the binomial probability density function is

$$
\begin{aligned}
f\left(y_{i}\right)= & \left(\begin{array}{c}
n_{i} \\
y_{i}
\end{array}\right) p_{i}{ }^{y_{i}}\left(1-p_{i}\right)^{n_{i}-y_{i}} \\
& =\left(\begin{array}{c}
n_{i} \\
y_{i}
\end{array}\right)\left(\frac{p_{i}}{1-p_{i}}\right)^{y_{i}}\left(1-p_{i}\right)^{n_{i}}
\end{aligned}
$$

where $y_{i}=0,1, \ldots, n_{i}$. The mean and variance can be obtained as

$$
E\left(Y_{i}\right)=\mu_{i}=n_{i} p_{i}
$$

and

$$
\operatorname{var}\left(Y_{i}\right)={\sigma_{i}^{2}}^{2}=n_{i} p_{i}\left(1-p_{i}\right)
$$

Again, the mean and variance depend on the underlying parameter $p_{i}$.

Suppose that the logit of the underlying probability $p_{i}$ is a linear function of the predictors. The systematic structure of the model can be shown as

$$
\operatorname{logit}\left(p_{i}\right)=\mathbf{x}_{i}^{t} \boldsymbol{\beta}
$$

where $x_{i}$ is a vector of covariates and $\boldsymbol{\beta}$ is a vector of regression coefficients.

The model defined in Eq.(4.2) and Eq.(4.4) is the generalized linear model with binomial response and logit link. Taking the exponential in Eq.(4.4), the odds for the $i$ th unit are

$$
\frac{p_{i}}{1-p_{i}}=\exp \left\{\mathbf{x}_{i}^{t} \boldsymbol{\beta}\right\}
$$


Solving Eq.(4.5) for the probability $p_{i}$ in the logit model, we get

$$
p_{i}=\frac{\exp \left\{\mathbf{x}_{i}{ }^{t} \boldsymbol{\beta}\right\}}{1+\exp \left\{\mathbf{x}_{i}{ }^{t} \boldsymbol{\beta}\right\}}=\frac{e^{\mathbf{x}_{i}{ }^{t} \boldsymbol{\beta}}}{1+e^{\mathbf{x}_{i}{ }^{t} \boldsymbol{\beta}}} .
$$

There is no explicit solution for the individual effects of predictors, as the right-hand side is a non-linear function of the predictors. To obtain an approximate answer, we can take derivatives with respect to $x_{j}$, that is

$$
\frac{d p_{i}}{d x_{i j}}=\beta_{j} p_{i}\left(1-p_{i}\right)
$$

Thus, the effect of the $j$ th predictor on the probability $p_{i}$ depends on the coefficient $\beta_{j}$ and the value of the probability.

\subsubsection{Maximum Likelihood Estimation}

For $n$ independent binomial observations, the likelihood function is a product of densities given by Eq.(4.3). The likelihood function of $\boldsymbol{\beta}$ for given data $\left(y_{i}, n_{i}, x_{i}\right)$ is

$$
L=\prod_{i=1}^{n}\left(\begin{array}{l}
n_{i} \\
y_{i}
\end{array}\right) p_{i}{ }^{y_{i}}\left(1-p_{i}\right)^{n_{i}-y_{i}}
$$

where $p_{i}$ depends on the covariates $x_{i}$ and a vector $\boldsymbol{\beta}$ of $p$ parameters. The maximum likelihood estimates are the values for $\boldsymbol{\beta}$ which maximize the likelihood function (4.6). Taking logarithms on both sides of Eq.(4.6), we get the log-likelihood function

$$
\begin{aligned}
\log L= & \prod_{i=1}^{n} \log \left(\begin{array}{c}
n_{i} \\
y_{i}
\end{array}\right) p_{i}{ }^{y_{i}}\left(1-p_{i}\right)^{n_{i}-y_{i}} \\
& =\sum_{i=1}^{n}\left\{\log \left(\begin{array}{c}
n_{i} \\
y_{i}
\end{array}\right)+y_{i} \log \frac{p_{i}}{1-p_{i}}+n_{i} \log \left(1-p_{i}\right)\right\} .
\end{aligned}
$$


To obtain the score equation, we take the first derivative of Eq.(4.7) with respect to $\boldsymbol{\beta}$. That is,

$$
\begin{aligned}
\frac{\partial \log L}{\partial \boldsymbol{\beta}}= & \sum_{i=1}^{n}\left\{y_{i} x_{i}-n_{i} \frac{1}{1-p_{i}} \frac{\partial p_{i}}{\partial \boldsymbol{\beta}}\right\} \\
& =\sum_{i=1}^{n}\left\{y_{i} x_{i}-n_{i} \frac{1}{1-p_{i}} p_{i}\left(1-p_{i}\right) x_{i}\right\} \\
& =\sum_{i=1}^{n}\left\{y_{i} x_{i}-n_{i} p_{i} x_{i}\right\} \\
& =\sum_{i=1}^{n}\left(y_{i}-n_{i} p_{i}\right) x_{i} .
\end{aligned}
$$

The maximum likelihood estimators of $\boldsymbol{\beta}$ are obtained by solving the ML estimating equations, which can be written as

$$
\sum_{i=1}^{n}\left(y_{i}-n_{i} \hat{p}_{i}\right) x_{i}=0
$$

Here, $\hat{\boldsymbol{\beta}}$ cannot be obtained explicitly. Some numerical algorithm such as the iteratively reweighted least squares (IRWLS) or Newton-Raphson method can be used to solve the likelihood equations.

\subsection{Simulation Study}

To assess the performance of the maximum likelihood method, we ran sixteen sets of simulations under four scenarios using the binary regression model with missing covariates. In the first two scenarios, the estimates were studied under correctly specified MAR models and NMAR models, respectively. In the second two scenarios, the estimates were studied under misspecified models for the missing data. We investigated 
the empirical properties of the ML estimators of $\hat{\boldsymbol{\beta}}$ which may be adequate to allow the inferential procedures under normal theory for moderate sample sizes.

We computed $95 \%$ confidence intervals for the regression coefficients as well as for the nuisance parameters for each of the sixteen sets of simulations to investigate if departures from normality are sufficiently severe to adversely affect normal-theory parametric inferences. If the normality assumption is satisfied, then $t=\left(\hat{\theta_{n}}-\theta\right) /$ s.e. $\left(\hat{\theta_{n}}\right)$ approximately follows a Student's $t_{n-p}$ distribution, where $n$ is the total number of observations in the data set and $p$ is the number of parameters in the model. Then the nominal level of the confidence interval for $\theta$ is $100(1-\alpha)$ as obtained from the confidence interval $\hat{\theta_{n}} \pm t_{n-p ; \alpha / 2}$ s.e. $\left(\hat{\theta_{n}}\right)$.

\subsubsection{Investigative Methods}

We studied the empirical properties of the maximum likelihood estimators in terms of biases and mean squared errors (MSEs) of the estimators, as well as coverage probabilities (CPs) and average lengths of the individual confidence intervals for the regression parameters.

Bias: The bias of an estimator $\hat{\theta}$ of a parameter $\theta$ is obtained as the difference between the expected value of $\hat{\theta}$ and the true value of the parameter $\theta$, given by

$$
\begin{aligned}
\operatorname{Bias}(\hat{\theta}) & =E(\hat{\theta})-\theta \\
& \approx\left\{\frac{1}{S} \sum_{s=1}^{S} \hat{\theta}^{(s)}\right\}-\theta
\end{aligned}
$$


where $\hat{\theta}^{(s)}$ is the estimate of $\theta$ obtained at the $s^{\text {th }}$ simulation and $S$ is the simulation size.

Mean Square Error: The mean squared error (MSE) of an estimator $\hat{\theta}$ of a parameter $\theta$ can be obtained as

$$
\begin{aligned}
\operatorname{MSE}(\hat{\theta}) & =E(\hat{\theta}-\theta)^{2} \\
& \approx \frac{1}{S} \sum_{s=1}^{S}\left(\hat{\theta}^{(s)}-\theta\right)^{2}
\end{aligned}
$$

where $\hat{\theta}^{(s)}$ is the estimate of $\theta$ obtained at the $s^{t h}$ simulation and $S$ is the simulation size.

Coverage Probability: The coverage probability of an estimator $\hat{\theta}$ for a $95 \%$ confidence interval on $\theta$ is obtained from

$$
C P(\hat{\theta})=\frac{1}{S} \sum_{s=1}^{S} I\left\{\left|\hat{\theta}^{(s)}-\theta\right| \leq 1.96 \times \operatorname{SE}\left(\hat{\theta}^{(s)}\right)\right\}
$$

where $\operatorname{SE}(\hat{\theta})$ is an estimate of the standard errors of $\hat{\theta}$ and $\mathrm{I}\{\}$ is an indicator variable.

The $95 \%$ confidence interval for $\theta$ is obtained as

$$
C I(\hat{\theta})=\hat{\theta} \pm 1.96 \times \mathrm{SE}(\hat{\theta})
$$

Average Length: The length of a confidence interval for $\theta$ is obtained as the difference between two confidence limits:

$$
\mathrm{L}^{c}(\hat{\theta})=\{\hat{\theta}+1.96 \times \mathrm{SE}(\hat{\theta})\}-\{\hat{\theta}-1.96 \times \mathrm{SE}(\hat{\theta})\}
$$


The the average length is obtained by

$$
\operatorname{Ave} . \mathrm{L}^{c}(\hat{\theta})=\frac{1}{S} \sum_{s=1}^{S} \mathrm{~L}^{c}\left(\hat{\theta}^{(s)}\right)
$$

where $S$ is the simulation size.

\subsubsection{Binary Logistic Model for Simulation}

Consider a binary-logit model with two continuous covariates $x_{1}$ and $x_{2}$. The covariates $\left(x_{i 1}, x_{i 2}\right)$ for the $i$ th individual are assumed to be independent normal with mean $\boldsymbol{\mu}_{x}$ and covariance matrix $\boldsymbol{\Sigma}_{x}$. For the $i$ th response variable $y_{i}$, assume

$$
y_{i} \mid x_{1 i}, x_{2 i} \sim \text { independent Bernoulli }\left(\mu_{i}\right), i=1,2 \ldots n
$$

and

$$
\theta_{i}=\operatorname{logit}\left(\mu_{i}\right)=\log \left\{\mu_{i} /\left(1-\mu_{i}\right)\right\}=\mathbf{x}_{i}{ }^{t} \boldsymbol{\beta}=\beta_{0}+\beta_{1} x_{i 1}+\beta_{2} x_{i 2}
$$

In this setting, we have $\mu_{i}=\mu_{i}\left(\boldsymbol{\beta}, \mathbf{x}_{i}\right)=E\left\{y_{i} \mid \mathbf{x}_{i}, \boldsymbol{\beta}\right\}=\exp \left(\mathbf{x}_{i}{ }^{t} \boldsymbol{\beta}\right) /\left\{1+\exp \left(\mathbf{x}_{i}^{t} \boldsymbol{\beta}\right)\right\}$ and $\operatorname{var}\left\{y_{i} \mid \mathbf{x}_{i}, \boldsymbol{\beta}\right\}=\sigma_{i}^{2}\left(\boldsymbol{\beta}, \mathbf{x}_{i}\right)=\exp \left(\mathbf{x}_{i}^{t} \boldsymbol{\beta}\right) /\left\{1+\exp \left(\mathbf{x}_{i}{ }^{t} \boldsymbol{\beta}\right)\right\}^{2}$. Also, assume a logistic regression model for the missing data mechanism in the form

$$
f_{v_{2 i} \mid y_{i}, x_{1 i}, x_{2 i}}\left(v_{2 i} \mid y_{i}, x_{1 i}, x_{2 i}, \boldsymbol{\tau}\right)=\pi_{i}^{v_{2 i}}\left(1-\pi_{i}\right)^{\left(1-v_{2 i}\right)}
$$

$$
\operatorname{logit}\left(\pi_{i}\right)=\operatorname{logit}\left\{P\left(v_{2 i}=1 \mid y_{i}, x_{1 i}, x_{2 i}, \boldsymbol{\tau}\right)\right\}=\tau_{0}+\tau_{1} x_{1 i}+\tau_{2} x_{2 i}+\tau_{3} y_{i}
$$


where the values of the covariate $x_{1}$ are completely observed $\left(v_{1 i}=0\right.$ for all $\left.i\right)$ and the values of $x_{2}$ are missing according to the missing data model (4.10).

The following methods are used in the simulations:

MAR: When the data are generated by considering the missing data mechanism $\tau_{3}=0$ and the regression coefficients $\boldsymbol{\beta}$ as well as the nuisance parameters $\boldsymbol{\alpha}$ of the covariate distribution are estimated by considering $\tau_{3}=0$.

NMAR: When the data are generated by considering the missing data mechanism $\tau_{3} \neq 0$ and the regression coefficients $\boldsymbol{\beta}$ as well as the nuisance parameters $\boldsymbol{\alpha}$ of the covariate distribution are estimated by considering $\tau_{3} \neq 0$.

Correctly Specified: When the data sets are generated either considering the MAR method and the estimates are obtained under the MAR method or the data sets are generated by considering the NMAR method and the estimates are obtained under the NMAR method.

Misspecified: When the data sets are generated either considering the MAR method and the estimates are obtained under the NMAR method or the data sets are generated by considering the NMAR method and the estimates are obtained under the MAR method.

For the binary regression, a series of 1000 data were generated from the binary 
model (4.9) when the sample sizes are $n=100$ and 200. We generated the values of the covariates $\left(x_{i 1}, x_{i 2}\right)$ from a bivariate normal distribution with mean $\boldsymbol{\mu}_{x}=(2,1)^{t}$ and covariance matrix

$$
\boldsymbol{\Sigma}_{x}=\left(\begin{array}{ll}
1 & .2 \\
.2 & 1
\end{array}\right)
$$

In our notation, $\boldsymbol{\alpha}=\left(\mu_{x_{1}}, \mu_{x_{2}}, \sigma_{x_{1}}^{2}, \sigma_{x_{2}}^{2}, \sigma_{x_{1} x_{2}}\right)^{t}=(2,1,1,1,0.2)^{t}$. The regression coefficients were fixed at $\boldsymbol{\beta}=(-2,1,0.5)^{t}$ and $\boldsymbol{\beta}=(-2,0.5,1)^{t}$.

We obtain the ML estimates of the regression parameters $\boldsymbol{\beta}$ as well as the nuisance parameters $\boldsymbol{\alpha}$ and $\boldsymbol{\tau}$ by the iterative Newton-Raphson method described earlier.

\subsubsection{Results and Discussion for the Binary Model}

In this section, we studied empirical biases and mean square errors of the ML estimators under four scenarios, where each scenario contains four sets of simulations conducted under the binary regression model with missing covariates. We also computed $95 \%$ confidence intervals and average lengths of the confidence intervals for the regression coefficients $\boldsymbol{\beta}$ as well as the nuisance parameters $\boldsymbol{\tau}$ of the missing data mechanism for different sample sizes.

When the missing data mechanism is MAR, for the two choices of $\boldsymbol{\beta}=(-2,1,0.5)^{t}$ and $\boldsymbol{\beta}=(-2,0.5,1)^{t}$, the data contained roughly $34.8 \%$ and $33.7 \%$ missing values, respectively. On the other hand, when the missing data mechanism is NMAR, for the two choices of $\boldsymbol{\beta}=(-2,1,0.5)^{t}$ and $\boldsymbol{\beta}=(-2,0.5,1)^{t}$, the data contained roughly $44.2 \%$ and $40 \%$ missing values, respectively. The simulation results are discussed here under four scenarios as presented bellow. 
Table 4.1: Empirical biases and mean squared errors (MSEs) of maximum likelihood estimators in binary logistic regression models with missing covariates for different sample sizes. Slope parameter $\beta_{1}=1$. Correctly specified MAR model is assumed for missing data.

\begin{tabular}{cccccc}
\hline & & \multicolumn{2}{c}{$\mathrm{n}=100$} & \multicolumn{2}{c}{$\mathrm{n}=200$} \\
\hline Parameter & True value & Bias & MSE & Bias & MSE \\
\hline$\beta_{0}$ & -2 & -0.13830 & 0.49592 & -0.06633 & 0.21766 \\
$\beta_{1}$ & 1 & 0.13338 & 0.12268 & 0.09268 & 0.05195 \\
$\beta_{2}$ & 0.5 & 0.03445 & 0.12408 & 0.00766 & 0.05328 \\
$\mu_{1}$ & 2 & -0.00264 & 0.01069 & 0.00069 & 0.00502 \\
$\mu_{2}$ & 1 & -0.25879 & 0.08037 & -0.25189 & 0.07036 \\
$\sigma_{1}^{2}$ & 1 & -0.00952 & 0.01949 & 0.00013 & 0.01039 \\
$\sigma_{2}^{2}$ & 1 & -0.15111 & 0.04830 & -0.15835 & 0.03964 \\
$\sigma_{12}$ & 0.2 & -0.13801 & 0.03454 & -0.13462 & 0.02640 \\
\hline
\end{tabular}

Scenario 1: True model: $\operatorname{MAR}\left(\tau_{3}=0\right)$; fitted model: $\operatorname{MAR}\left(\tau_{3}=0\right)$.

In this scenario, the data were generated by using the MAR model, where the parameters of the missing-data mechanism were chosen as $\boldsymbol{\tau}=(-4,1,1,0)^{t}$ and the data were fitted by using the MAR model as well. In that sense, the model is correctly specified. The regression coefficients were fixed at $\boldsymbol{\beta}=(-2,1,0.5)^{t}$ and $\boldsymbol{\beta}=(-2,0.5,1)^{t}$. As the model is correctly specified, we expect to get negligible biases, small MSEs and good coverage probabilities for the estimators.

Table 4.1 presents the simulated biases and mean squared errors, and Table 4.2 presents the coverage probabilities and the mean lengths of the confidence intervals for different sample sizes, where the regression coefficients were fixed at $\boldsymbol{\beta}=(-2,1,0.5)^{t}$ 
Table 4.2: Coverage probabilities (CPs), average lengths of maximum likelihood estimators in binary logistic regression models with missing covariates for different sample sizes. Slope parameter $\beta_{1}=1$. Correctly specified MAR model is assumed for missing data.

\begin{tabular}{cccccc}
\hline & & \multicolumn{2}{c}{$\mathrm{n}=100$} & \multicolumn{2}{c}{$\mathrm{n}=200$} \\
\hline Parameter & True value & $\mathrm{CP}$ & Length & $\mathrm{CP}$ & Length \\
\hline$\beta_{0}$ & -2 & 0.95401 & 2.64735 & 0.95910 & 1.80479 \\
$\beta_{1}$ & 1 & 0.94652 & 1.19274 & 0.94581 & 0.80706 \\
$\beta_{2}$ & 0.5 & 0.95401 & 1.29181 & 0.95092 & 0.88247 \\
\hline
\end{tabular}

Table 4.3: Empirical biases and mean squared errors (MSEs) of maximum likelihood estimators in binary logistic regression models with missing covariates for different sample sizes. Slope parameter $\beta_{1}=0.5$. Correctly specified MAR model is assumed for missing data.

\begin{tabular}{cccccc}
\hline & & \multicolumn{2}{c}{$\mathrm{n}=100$} & \multicolumn{2}{c}{$\mathrm{n}=200$} \\
\hline Parameter & True value & Bias & MSE & Bias & MSE \\
\hline$\beta_{0}$ & -2 & -0.15705 & 0.56732 & -0.06617 & 0.23958 \\
$\beta_{1}$ & 0.5 & 0.15070 & 0.10462 & 0.12908 & 0.053273 \\
$\beta_{2}$ & 1 & 0.08728 & 0.16743 & 0.03122 & 0.07741 \\
$\mu_{1}$ & 2 & 0.00533 & 0.010915 & 0.00110 & 0.00479 \\
$\mu_{2}$ & 1 & -0.22314 & 0.07167 & -0.22548 & 0.05755 \\
$\sigma_{1}^{2}$ & 1 & -0.01026 & 0.02384 & -0.00662 & 0.0098 \\
$\sigma_{2}^{2}$ & 1 & -0.15040 & 0.05244 & -0.14562 & 0.03354 \\
$\sigma_{12}$ & 0.2 & -0.11989 & 0.02844 & -0.11791 & 0.02175 \\
\hline
\end{tabular}


Table 4.4: Coverage probabilities (CPs), average lengths of maximum likelihood estimators in binary logistic regression models with missing covariates for different sample sizes. Slope parameter $\beta_{1}=0.5$. Correctly specified MAR model is assumed for missing data.

\begin{tabular}{cccccc}
\hline & & \multicolumn{2}{c}{$\mathrm{n}=100$} & \multicolumn{2}{c}{$\mathrm{n}=200$} \\
\hline Parameter & True value & $\mathrm{CP}$ & Length & $\mathrm{CP}$ & Length \\
\hline$\beta_{0}$ & -2 & 0.95685 & 2.73537 & 0.95563 & 1.85475 \\
$\beta_{1}$ & 0.5 & 0.93851 & 1.07735 & 0.91022 & 0.73181 \\
$\beta_{2}$ & 1 & 0.95793 & 1.47877 & 0.94634 & 0.99954 \\
\hline
\end{tabular}

and the parameters of the missing-data mechanism were chosen as $\boldsymbol{\tau}=(-4,1,1,0)^{t}$.

On the other hand, to study the impact of changes to the coefficients of missing covariates, we consider the regression coefficients $\boldsymbol{\beta}=(-2,0.5,1)^{t}$ keeping the nuisance parameters $\boldsymbol{\alpha}$ of the covariate distribution and $\boldsymbol{\tau}$ of the missing data mechanism similar to those as used for correctly specified models under the MAR setting.

Table 4.3 presents the simulated biases and mean squared errors, and Table 4.4 presents the coverage probabilities and mean lengths of the confidence intervals for different sample sizes under the same setting as above but the regression coefficients were fixed at $\boldsymbol{\beta}=(-2,0.5,1)^{t}$.

The maximum likelihood method appears to perform well for the correctly specified MAR mechanism under different sample sizes. The method provides small biases and mean squared errors for all the regression coefficients $\boldsymbol{\beta}$ as well as the nuisance parameters $\boldsymbol{\alpha}$ of the covariate distribution in both Tables 4.1 and 4.3. The confidence intervals 
have good coverages, which are close to the nominal $95 \%$ confidence level.

As expected, the biases and mean squared errors for all the regression coefficients $\boldsymbol{\beta}$ as well as the nuisance parameters $\boldsymbol{\alpha}$ become smaller when we increase the sample size. The coverage probabilities for the parameter estimates become closer to the nominal levels and the mean lengths based on confidence interval become smaller when we increase the sample size. The confidence intervals for the regression coefficients $\boldsymbol{\beta}$ slightly lose coverages due to the choice of different values of $\boldsymbol{\beta}=(-2,0.5,1)^{t}$. As shown in Table $4.4, \beta_{1}$ loses its coverage to 0.91022 from 0.94581 when the sample size is $n=200$, but it still has the good coverages. The slight loss of coverages is due to the small bias in estimation under moderate sample sizes. For larger sample sizes, the bias decreases and coverage probability improves.

Scenario 2: True model: $\operatorname{NMAR}\left(\tau_{3}=1\right)$; fitted model: $\operatorname{NMAR}\left(\tau_{3} \neq 0\right)$. In this scenario, the data were generated by using the NMAR model where the parameters of the missing data mechanism were chosen as $\boldsymbol{\tau}=(-4,1,1,1)^{t}$ and the data were fitted by using the NMAR model as well. In this case, the model is correctly specified. The regression coefficients were fixed at $\boldsymbol{\beta}=(-2,1,0.5)^{t}$ and $\boldsymbol{\beta}=(-2,0.5,1)^{t}$. Here we expect to get unbiased and efficient estimators.

Table 4.5 presents the simulated biases and mean squared errors and Table 4.6 presents the coverage probabilities and the mean lengths of the confidence intervals for different sample sizes, where the regression coefficients were fixed at $\boldsymbol{\beta}=(-2,1,0.5)^{t}$ and the parameters of the missing-data model were chosen as $\boldsymbol{\tau}=(-4,1,1,1)^{t}$. 
Table 4.5: Empirical biases and mean squared errors (MSEs) of maximum likelihood estimators in binary logistic regression models with missing covariates for different sample sizes. Slope parameter $\beta_{1}=1$. Correctly specified NMAR model is assumed for missing data.

\begin{tabular}{cccccc}
\hline & & \multicolumn{2}{c}{$\mathrm{n}=100$} & \multicolumn{2}{c}{$\mathrm{n}=200$} \\
\cline { 2 - 5 } Parameter & True value & Bias & MSE & Bias & MSE \\
\hline$\beta_{0}$ & -2 & -0.13911 & 0.56093 & -0.05877 & 0.20660 \\
$\beta_{1}$ & 1 & 0.05266 & 0.10679 & 0.019907 & 0.04151 \\
$\beta_{2}$ & 0.5 & 0.03752 & 0.11575 & 0.02235 & 0.04981 \\
$\tau_{0}$ & -4 & -0.70373 & 4.25333 & -0.30515 & 1.05603 \\
$\tau_{1}$ & 1 & 0.13530 & 0.21796 & 0.05548 & 0.08324 \\
$\tau_{2}$ & 1 & 0.23324 & 0.54397 & 0.12389 & 0.19967 \\
$\tau_{3}$ & 1 & 0.09170 & 1.28212 & 0.01686 & 0.19807 \\
$\mu_{1}$ & 2 & 0.00281 & 0.01061 & 0.00045 & 0.00521 \\
$\mu_{2}$ & 1 & 0.02093 & 0.02167 & 0.01216 & 0.01187 \\
$\sigma_{1}^{2}$ & 1 & 0.00268 & 0.02095 & 0.00183 & 0.00999 \\
$\sigma_{2}^{2}$ & 1 & 0.03100 & 0.05036 & 0.01929 & 0.02174 \\
$\sigma_{12}$ & 0.2 & 0.00675 & 0.01867 & 0.01053 & 0.01006 \\
\hline
\end{tabular}


Table 4.6: Coverage probabilities (CPs), average lengths of maximum likelihood estimators in binary logistic regression models with missing covariates for different sample sizes. Slope parameter $\beta_{1}=1$. Correctly specified NMAR model is assumed for missing data.

\begin{tabular}{cccccc}
\hline & & \multicolumn{2}{c}{$\mathrm{n}=100$} & \multicolumn{2}{c}{$\mathrm{n}=200$} \\
\hline Parameter & True value & $\mathrm{CP}$ & Length & $\mathrm{CP}$ & Length \\
\hline$\beta_{0}$ & -2 & 0.94769 & 2.61472 & 0.967 & 1.78360 \\
$\beta_{1}$ & 1 & 0.94869 & 1.16784 & 0.966 & 0.79773 \\
$\beta_{2}$ & 0.5 & 0.94668 & 1.21611 & 0.944 & 0.83585 \\
$\tau_{0}$ & -4 & 0.96881 & 5.026281 & 0.943 & 3.15704 \\
$\tau_{1}$ & 1 & 0.95775 & 1.55262 & 0.952 & 1.00601 \\
$\tau_{2}$ & 1 & 0.95875 & 2.12204 & 0.941 & 1.37445 \\
$\tau_{3}$ & 1 & 0.95573 & 2.54862 & 0.957 & 1.68455 \\
\hline
\end{tabular}

On the other hand, to study the impact of changes to the coefficients of missing covariates, we consider the regression coefficients $\boldsymbol{\beta}=(-2,0.5,1)^{t}$ keeping the nuisance parameters $\boldsymbol{\alpha}$ of the covariate distribution and $\boldsymbol{\tau}$ of the missing data mechanism similar to those as used for correctly specified models under the NMAR setting.

Table 4.7 presents the simulated biases and mean squared errors and Table 4.8 presents the coverage probabilities and the mean lengths of the confidence intervals for different sample sizes under the same setting but the regression coefficients were fixed at $\boldsymbol{\beta}=(-2,0.5,1)^{t}$.

The maximum likelihood method appears to perform well for the correctly specified model under the NMAR mechanism for different sample sizes. The method provides 
Table 4.7: Empirical biases and mean squared errors (MSEs) of maximum likelihood estimators in binary logistic regression models with missing covariates for different sample sizes. Slope parameter $\beta_{1}=0.5$. Correctly specified NMAR model is assumed for missing data.

\begin{tabular}{cccccc}
\hline & & \multicolumn{2}{c}{$\mathrm{n}=100$} & \multicolumn{2}{c}{$\mathrm{n}=200$} \\
\cline { 2 - 5 } Parameter & True value & Bias & MSE & Bias & MSE \\
\hline$\beta_{0}$ & -2 & -0.17290 & 0.65864 & -0.06385 & 0.24297 \\
$\beta_{1}$ & 0.5 & 0.03860 & 0.09034 & 0.00566 & 0.03502 \\
$\beta_{2}$ & 1 & 0.07162 & 0.17567 & 0.03740 & 0.07135 \\
$\tau_{0}$ & -4 & -0.68744 & 3.22699 & -0.38205 & 1.13386 \\
$\tau_{1}$ & 1 & 0.13598 & 0.21250 & 0.07739 & 0.08731 \\
$\tau_{2}$ & 1 & 0.26980 & 0.67595 & 0.13740 & 0.23439 \\
$\tau_{3}$ & 1 & -0.01712 & 0.51816 & 0.00523 & 0.20235 \\
$\mu_{1}$ & 2 & $-4.8048 \mathrm{E}-05$ & 0.00965 & 0.00132 & 0.00488 \\
$\mu_{2}$ & 1 & 0.02246 & 0.02071 & 0.01498 & 0.01216 \\
$\sigma_{1}^{2}$ & 1 & 0.00507 & 0.02077 & 0.00452 & 0.00964 \\
$\sigma_{2}^{2}$ & 1 & 0.04821 & 0.05101 & 0.02184 & 0.02353 \\
$\sigma_{12}$ & 0.2 & 0.01728 & 0.01878 & 0.00399 & 0.00966 \\
\hline
\end{tabular}


Table 4.8: Coverage probabilities (CPs), average lengths of maximum likelihood estimators in binary logistic regression models with missing covariates for different sample sizes. Slope parameter $\beta_{1}=0.5$. Correctly specified NMAR model is assumed for missing data.

\begin{tabular}{cccccc}
\hline & & \multicolumn{2}{c}{$\mathrm{n}=100$} & \multicolumn{2}{c}{$\mathrm{n}=200$} \\
\hline Parameter & True value & $\mathrm{CP}$ & Length & $\mathrm{CP}$ & Length \\
\hline$\beta_{0}$ & -2 & 0.94294 & 2.71037 & 0.955 & 1.84205 \\
$\beta_{1}$ & 0.5 & 0.94795 & 1.05521 & 0.951 & 0.71442 \\
$\beta_{2}$ & 1 & 0.93794 & 1.42141 & 0.946 & 0.97385 \\
$\tau_{0}$ & -4 & 0.97498 & 5.02201 & 0.955 & 3.18308 \\
$\tau_{1}$ & 1 & 0.96397 & 1.50315 & 0.94 & 0.97763 \\
$\tau_{2}$ & 1 & 0.96096 & 2.28808 & 0.927 & 1.48164 \\
$\tau_{3}$ & 0 & 0.94595 & 2.50098 & 0.945 & 1.65639 \\
\hline
\end{tabular}

unbiased estimators and small mean squared errors for all the regression coefficients $\boldsymbol{\beta}$ as well as the nuisance parameters $\boldsymbol{\alpha}$ of the covariate distribution. But estimates of $\boldsymbol{\tau}$ for the missing data mechanism show slightly large biases in both Tables 4.5 and 4.7. The parameter estimates have good coverages which are close to the nominal level. Here $\tau_{2}$ loses slight coverages with the value of 0.927 , as shown in Table 4.8 when the sample size is $n=200$.

As expected, the biases and mean squared errors for all the regression coefficients $\boldsymbol{\beta}$ as well as the nuisance parameters $\boldsymbol{\alpha}$ and $\boldsymbol{\tau}$ become smaller when we increase the sample size. The coverage probabilities for the parameter estimates become closer to the nominal levels and the mean lengths based on confidence intervals become smaller when we increase the sample size. 
Table 4.9: Empirical biases and mean squared errors (MSEs) of maximum likelihood estimators in binary logistic regression models with missing covariates for different sample sizes. Slope parameter $\beta_{1}=1$. Misspecified NMAR model is assumed for missing data.

\begin{tabular}{cccccc}
\hline & & \multicolumn{2}{c}{$\mathrm{n}=100$} & \multicolumn{2}{c}{$\mathrm{n}=200$} \\
\hline Parameter & True value & Bias & MSE & Bias & MSE \\
\hline$\beta_{0}$ & -2 & -0.11786 & 0.52308 & -0.09645 & 0.24979 \\
$\beta_{1}$ & 1 & 0.05453 & 0.10202 & 0.04459 & 0.05005 \\
$\beta_{2}$ & 0.5 & 0.02849 & 0.09784 & 0.01212 & 0.04478 \\
$\tau_{0}$ & -4 & -0.80274 & 4.07318 & -0.42001 & 1.30592 \\
$\tau_{1}$ & 1 & 0.14492 & 0.24338 & 0.08468 & 0.09054 \\
$\tau_{2}$ & 1 & 0.28534 & 0.74611 & 0.13966 & 0.27604 \\
$\tau_{3}$ & 0 & -0.04386 & 0.55968 & -0.04802 & 0.26663 \\
$\mu_{1}$ & 2 & -0.00016 & 0.010008 & -0.00113 & 0.00502 \\
$\mu_{2}$ & 1 & 0.01652 & 0.02074 & 0.00961 & 0.01149 \\
$\sigma_{1}^{2}$ & 1 & 0.00174 & 0.02050 & -0.00514 & 0.01008 \\
$\sigma_{2}^{2}$ & 1 & 0.02826 & 0.04614 & 0.02421 & 0.02519 \\
$\sigma_{12}$ & 0.2 & 0.00267 & 0.01865 & 0.00564 & 0.00899 \\
\hline
\end{tabular}

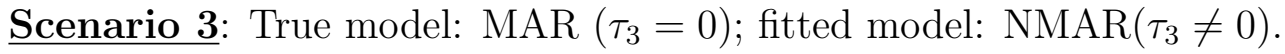

In this scenario, the data were generated by using the MAR model where the parameters of the missing-data mechanism were chosen as $\boldsymbol{\tau}=(-4,1,1,0)^{t}$ and the data were fitted by using the NMAR model $\boldsymbol{\tau}=(-4,1,1,1)^{t}$. In this case, the model is misspecified. The regression coefficients were fixed at $\boldsymbol{\beta}=(-2,1,0.5)^{t}$ and $\boldsymbol{\beta}=(-2,1,0.5)^{t}$. Here we expect to get larger biases, MSEs and bad coverages for the estimators under the misspecified model as compared to the correctly specified models.

Table 4.9 presents the simulated biases and mean squared errors and Table 4.10 presents the coverage probabilities and the mean lengths of the confidence intervals for 
Table 4.10: Coverage probabilities (CPs), average lengths of maximum likelihood estimators in binary logistic regression models with missing covariates for different sample sizes. Slope parameter $\beta_{1}=1$. Misspecified NMAR model is assumed for missing data.

\begin{tabular}{cccccc}
\hline & & \multicolumn{2}{c}{$\mathrm{n}=100$} & \multicolumn{2}{c}{$\mathrm{n}=200$} \\
\hline Parameter & True value & $\mathrm{CP}$ & Length & $\mathrm{CP}$ & Length \\
\hline$\beta_{0}$ & -2 & 0.94183 & 2.57221 & 0.938 & 1.77996 \\
$\beta_{1}$ & 1 & 0.94784 & 1.15772 & 0.941 & 0.80284 \\
$\beta_{2}$ & 0.5 & 0.95687 & 1.16200 & 0.951 & 0.80151 \\
$\tau_{0}$ & -4 & 0.96891 & 5.36726 & 0.953 & 3.33533 \\
$\tau_{1}$ & 1 & 0.95487 & 1.58073 & 0.943 & 1.02386 \\
$\tau_{2}$ & 1 & 0.95988 & 2.33606 & 0.922 & 1.49519 \\
$\tau_{3}$ & 0 & 0.96891 & 2.74035 & 0.941 & 1.82256 \\
\hline
\end{tabular}

different sample sizes, where the regression coefficients were fixed at $\boldsymbol{\beta}=(-2,1,0.5)^{t}$ and the parameters of the missing-data mechanism were chosen as $\boldsymbol{\tau}=(-4,1,1,0)^{t}$.

On the other hand, to study the impact of changes to the coefficients of missing covariates, we consider the regression coefficients $\boldsymbol{\beta}=(-2,0.5,1)^{t}$ keeping the nuisance parameters $\boldsymbol{\alpha}$ of the covariate distribution and $\boldsymbol{\tau}$ of the missing data mechanism similar to those as used for misspecified models under the same setting.

Table 4.11 presents the simulated biases and mean squared errors, and Table 4.12 presents the coverage probabilities and the mean lengths of the confidence intervals for different sample sizes under same setting, but the regression coefficients were fixed at $\boldsymbol{\beta}=(-2,0.5,1)^{t}$. 
Table 4.11: Empirical biases and mean squared errors (MSEs) of maximum likelihood estimators in binary logistic regression models with missing covariates for different sample sizes. Slope parameter $\beta_{1}=0.5$. Misspecified NMAR model is assumed for missing data.

\begin{tabular}{cccccc}
\hline & & \multicolumn{2}{c}{$\mathrm{n}=100$} & \multicolumn{2}{c}{$\mathrm{n}=200$} \\
\cline { 2 - 5 } Parameter & True value & Bias & MSE & Bias & MSE \\
\hline$\beta_{0}$ & -2 & -0.12326 & 0.53448 & -0.04423 & 0.22837 \\
$\beta_{1}$ & 0.5 & 0.03932 & 0.078824 & 0.01717 & 0.03534 \\
$\beta_{2}$ & 1 & 0.03724 & 0.14559 & 0.01346 & 0.06095 \\
$\tau_{0}$ & -4 & -0.87216 & 4.50733 & -0.34783 & 1.14227 \\
$\tau_{1}$ & 1 & 0.15858 & 0.23369 & 0.07499 & 0.07674 \\
$\tau_{2}$ & 1 & 0.32285 & 0.86813 & 0.11979 & 0.25882 \\
$\tau_{3}$ & 0 & -0.11847 & 0.75405 & -0.05043 & 0.26919 \\
$\mu_{1}$ & 2 & 0.00165 & 0.01039 & 0.00046 & 0.00528 \\
$\mu_{2}$ & 1 & 0.02229 & 0.02078 & 0.01005 & 0.00942 \\
$\sigma_{1}^{2}$ & 1 & -0.00656 & 0.02065 & -0.00326 & 0.01025 \\
$\sigma_{2}^{2}$ & 1 & 0.03859 & 0.05044 & 0.01707 & 0.02140 \\
$\sigma_{12}$ & 0.2 & 0.00537 & 0.01829 & 0.00265 & 0.00826 \\
\hline & & & & &
\end{tabular}


Table 4.12: Coverage probabilities (CPs), average lengths of maximum likelihood estimators in binary logistic regression models with missing covariates for different sample sizes. Slope parameter $\beta_{1}=0.5$. Misspecified NMAR model is assumed for missing data.

\begin{tabular}{cccccc}
\hline & & \multicolumn{2}{c}{$\mathrm{n}=100$} & \multicolumn{2}{c}{$\mathrm{n}=200$} \\
\hline Parameter & True value & $\mathrm{CP}$ & Length & $\mathrm{CP}$ & Length \\
\hline$\beta_{0}$ & -2 & 0.95436 & 2.61883 & 0.949 & 1.79373 \\
$\beta_{1}$ & 0.5 & 0.96045 & 1.03547 & 0.955 & 0.70848 \\
$\beta_{2}$ & 1 & 0.94118 & 1.32528 & 0.955 & 0.91731 \\
$\tau_{0}$ & -4 & 0.98174 & 5.38553 & 0.952 & 3.20270 \\
$\tau_{1}$ & 1 & 0.96450 & 1.53669 & 0.953 & 0.96499 \\
$\tau_{2}$ & 1 & 0.95740 & 2.46991 & 0.942 & 1.54279 \\
$\tau_{3}$ & 0 & 0.94929 & 2.78605 & 0.939 & 1.81549 \\
\hline
\end{tabular}

The maximum likelihood method still appears to perform well for the misspecified model under different sample sizes. The method still provides small biases and mean squared errors for all the regression coefficients $\boldsymbol{\beta}$ as well as the nuisance parameters $\boldsymbol{\alpha}$ of the covariate distribution. The method produces larger biases and mean squared errors for $\boldsymbol{\tau}$ of the missing data mechanism compared to Scenario 2 for both choices of $\boldsymbol{\beta}=(-2,1,0.5)^{t}$ and $\boldsymbol{\beta}=(-2,0.5,1)^{t}$. The parameter estimates have good coverages that are close to their nominal levels.

As expected, the biases and mean squared errors for all the regression coefficients $\boldsymbol{\beta}$ as well as the nuisance parameters $\boldsymbol{\alpha}$ become smaller when we increase sample size. Although biases are large for $\boldsymbol{\tau}$ with $\mathrm{n}=100$, they become smaller when we increase the sample size. The coverage probabilities for the parameter estimates become closer to the nominal levels, and mean lengths of confidence intervals become smaller when we 
Table 4.13: Empirical biases and mean squared errors (MSEs) of maximum likelihood estimators in binary logistic regression models with missing covariates for different sample sizes. Slope parameter $\beta_{1}=1$. Misspecified MAR model is assumed for missing data.

\begin{tabular}{cccccc}
\hline & & \multicolumn{2}{c}{$\mathrm{n}=100$} & \multicolumn{2}{c}{$\mathrm{n}=200$} \\
\hline Parameter & True value & Bias & MSE & Bias & MSE \\
\hline$\beta_{0}$ & -2 & -0.03009 & 0.55848 & 0.07051 & 0.22132 \\
$\beta_{1}$ & 1 & 0.14316 & 0.13654 & 0.09799 & 0.05506 \\
$\beta_{2}$ & 0.5 & -0.12182 & 0.16052 & -0.15326 & 0.08051 \\
$\mu_{1}$ & 2 & 0.00060 & 0.00970 & 0.00232 & 0.00513 \\
$\mu_{2}$ & 1 & -0.33276 & 0.12984 & -0.33644 & 0.12185 \\
$\sigma_{1}^{2}$ & 1 & -0.01189 & 0.01973 & -0.00161 & 0.00978 \\
$\sigma_{2}^{2}$ & 1 & -0.15891 & 0.05522 & -0.17576 & 0.048134 \\
$\sigma_{12}$ & 0.2 & -0.15666 & 0.04368 & -0.16947 & 0.03918 \\
\hline
\end{tabular}

increase the sample size. The estimates of regression coefficients $\boldsymbol{\beta}$ and nuisance parameters $\boldsymbol{\tau}$ lose small amount of coverages for the choice of $\boldsymbol{\beta}=(-2,1,0.5)^{t}$ as shown in Table 4.10 .

Scenario 4: True model: NMAR $\left(\tau_{3}=1\right)$; fitted model: MAR $\left(\tau_{3}=0\right)$. In this scenario, the data were generated by using the NMAR model where the parameters of the missing-data mechanism were chosen as $\boldsymbol{\tau}=(-4,1,1,1)^{t}$ and the data were fitted by using the MAR model $\boldsymbol{\tau}=(-4,1,1,0)^{t}$. In this case, the model is misspecified. The regression coefficients were fixed at $\boldsymbol{\beta}=(-2,1,0.5)^{t}$ and $\boldsymbol{\beta}=(-2,1,0.5)^{t}$.

Table 4.13 presents the simulated biases and mean squared errors, and Table 4.14 presents the coverage probabilities and the mean lengths of the confidence intervals for 
Table 4.14: Coverage probabilities (CPs), average lengths of maximum likelihood estimators in binary logistic regression models with missing covariates for different sample sizes. Slope parameter $\beta_{1}=1$. Misspecified MAR model is assumed for missing data.

\begin{tabular}{cccccc}
\hline & & \multicolumn{2}{c}{$\mathrm{n}=100$} & \multicolumn{2}{c}{$\mathrm{n}=200$} \\
\hline Parameter & True value & $\mathrm{CP}$ & Length & $\mathrm{CP}$ & Length \\
\hline$\beta_{0}$ & -2 & 0.94405 & 2.62845 & 0.94344 & 1.77738 \\
$\beta_{1}$ & 1 & 0.94286 & 1.19478 & 0.93810 & 0.80189 \\
$\beta_{2}$ & 0.5 & 0.91548 & 1.37637 & 0.89861 & 0.93329 \\
\hline
\end{tabular}

Table 4.15: Empirical biases and mean squared errors (MSEs) of maximum likelihood estimators in binary logistic regression models with missing covariates for different sample sizes. Slope parameter $\beta_{1}=0.5$. Misspecified MAR model is assumed for missing data.

\begin{tabular}{cccccc}
\hline & & \multicolumn{2}{c}{$\mathrm{n}=100$} & \multicolumn{2}{c}{$\mathrm{n}=200$} \\
\hline Parameter & True value & Bias & MSE & Bias & MSE \\
\hline$\beta_{0}$ & -2 & 0.02397 & 0.53173 & 0.12072 & 0.23781 \\
$\beta_{1}$ & 0.5 & 0.13680 & 0.10290 & 0.12353 & 0.04917 \\
$\beta_{2}$ & 1 & -0.06889 & 0.19882 & -0.13774 & 0.09662 \\
$\mu_{1}$ & 2 & -0.00035 & 0.010798 & 0.00428 & 0.00491 \\
$\mu_{2}$ & 1 & -0.28008 & 0.09548 & -0.28859 & 0.09117 \\
$\sigma_{1}^{2}$ & 1 & -0.00441 & 0.02022 & -0.00634 & 0.01041 \\
$\sigma_{2}^{2}$ & 1 & -0.17319 & 0.05536 & -0.18470 & 0.04887 \\
$\sigma_{12}$ & 0.2 & -0.12516 & 0.03246 & -0.14027 & 0.02935 \\
\hline
\end{tabular}


Table 4.16: Coverage probabilities (CPs), average lengths of maximum likelihood estimators in binary logistic regression models with missing covariates for different sample sizes. Slope parameter $\beta_{1}=0.5$. Misspecified MAR model is assumed for missing data.

\begin{tabular}{cccccc}
\hline & & \multicolumn{2}{c}{$\mathrm{n}=100$} & \multicolumn{2}{c}{$\mathrm{n}=200$} \\
\hline Parameter & True value & $\mathrm{CP}$ & Length & $\mathrm{CP}$ & Length \\
\hline$\beta_{0}$ & -2 & 0.94158 & 2.69793 & 0.92300 & 2.04204 \\
$\beta_{1}$ & 0.5 & 0.93471 & 1.06092 & 0.92405 & 0.80070 \\
$\beta_{2}$ & 1 & 0.92554 & 1.58067 & 0.88713 & 1.19307 \\
\hline
\end{tabular}

different sample sizes, where the regression coefficients were fixed at $\boldsymbol{\beta}=(-2,1,0.5)^{t}$ and the parameters of the missing-data mechanism were chosen as $\boldsymbol{\tau}=(-4,1,1,1)^{t}$.

On the other hand, to study the impact of changes to the coefficients of missing value covariates, we consider the regression coefficients $\boldsymbol{\beta}=(-2,0.5,1)^{t}$ keeping the nuisance parameters $\boldsymbol{\alpha}$ of the covariate distribution and $\boldsymbol{\tau}$ of the missing data mechanism similar to those as used for misspecified models under the same setting.

Table 4.15 presents the simulated biases and mean squared errors, and Table 4.16 presents the coverage probabilities and the mean lengths of the confidence intervals for different sample sizes under same setting, but the regression coefficients were fixed at $\boldsymbol{\beta}=(-2,0.5,1)^{t}$

The maximum likelihood method doesn't appear to perform well under this misspecified method for different sample sizes. The method provides slightly large biases and mean squared errors for all the regression coefficients $\boldsymbol{\beta}$ as well as the nuisance 
parameters $\boldsymbol{\alpha}$ of the covariate distribution. Also the coverages are not very close to the nominal level $95 \%$.

As expected, the biases and mean squared errors for all the regression coefficients $\boldsymbol{\beta}$ as well as the nuisance parameters $\boldsymbol{\alpha}$ become larger when we increase the sample sizes. The coverage probabilities for the parameter estimates slightly moved away from the nominal levels. The mean lengths based on confidence interval and coverage probabilities become smaller when we increase the sample size. The regression coefficients $\beta_{2}$ loses it's coverages for both choices of $\boldsymbol{\beta}=(-2,1,0.5)^{t}$ and $\boldsymbol{\beta}=(-2,0.5,1)^{t}$. As seen in Table 4.14 and 4.16 the coverages for $\beta_{2}$ decreases when the sample size increases from $\mathrm{n}=100$ to $\mathrm{n}=200$.

It is clear from above four scenarios that Scenario 2 and 3 provide approximately unbiased estimates of the regression coefficients $\boldsymbol{\beta}$ as well as the nuisance parameters $\boldsymbol{\alpha}$ of the covariate distribution and $\boldsymbol{\tau}$ of the missing data mechanism under all simulation configurations considered. On the other hand, Scenario 1 and 4 provide large biases of the estimators in both regression parameters and nuisance parameters. For example, in Tables 4.1 and 4.3, under correctly specified method, the estimator of $\beta_{1}$ gives large bias 0.13338 and 0.15070 respectively when $n=100$. As shown in Tables 4.13 and 4.15, under the misspecified method, the estimator of $\beta_{1}$ gives large biases 0.14316 and 0.13680 respectively when $n=100$.

When comparing four scenarios, our studied method works better for Scenario 2 and worst for Scenario 4. The Scenario 2 provides small biases for all the parameter 
estimates under the correctly specified model. For example, in Tables 4.5 and 4.7, the estimator of $\beta_{1}$ gives smaller biases 0.05266 and 0.03860 , respectively when $n=100$. Also, Scenario 3, provides small biases for all the parameter estimates under the misspecified model. For example, in Tables 4.9 and 4.11 , the estimator of $\beta_{1}$ gives smaller biases 0.05453 and 0.03932 respectively when $n=100$.

Scenario 2 also gives the better coverage probabilities and mean lengths for the regression coefficients $\boldsymbol{\beta}$ as well as the nuisance parameters $\boldsymbol{\tau}$ of the missing data mechanism as shown in Tables 4.6 and 4.8 under the correctly specified NMAR mechanism. As we expected, the coverage probabilities are very close to the nominal level $95 \%$ which gradually improve and the mean lengths gradually decrease when we increase the sample size for both choice of $\boldsymbol{\beta}=(-2,1,0.5)^{t}$ and $\boldsymbol{\beta}=(-2,0.5,1)^{t}$. Although Scenario 3 gives good coverages for all parameter estimates, it loses a small amount of coverages when we increase the sample size for the choice of $\boldsymbol{\beta}=(-2,0.5,1)^{t}$. The coverages decrease for both $\boldsymbol{\beta}$ and $\boldsymbol{\tau}$ accordingly with increased sample sizes.

Again, in Scenario 1, the estimators of $\beta_{1}$ gives empirical coverage probabilities of 0.94652 and 0.93851 respectively in Tables 4.2 and 4.4. In Scenario 4, the estimators of $\beta_{2}$ gives empirical coverage probabilities 0.91548 and 0.92554 in Tables 4.14 and 4.16 respectively, which are quite smaller than other scenarios.

Here we should note that there is no big bias for all estimates under the four scenarios. For the MSEs and mean lengths of the estimators of model parameters with different sample sizes, the empirical study shows that the MSEs and mean lengths decrease as 
the number of sample size $n$ increases. In terms of CPs, Scenarios 1, 2 and 3 give good coverages under both specified and misspecified models. 


\section{Chapter 5}

\section{Poisson Regression}

\subsection{Introduction}

In an experiment, under some ideal conditions, if independent successive events occur in the same rate, the Poisson model is appropriate for the number of events observed (McCullagh and Nelder, 1989). A Poisson regression model is sometimes known as a log-linear model. The log-linear model for Poisson regression is commonly used to model the mean response of counts as a function of covariates.

The analysis of count data dominates an important place in applied statistics in many areas. The Poisson regression is used to model count data. We experience count data when the outcome of interest takes only non-negative integer values, such as the number of children in a family, the number of accidents at 10 different intersections, the number of domestic violence incidents in a month, and so on. Also, we may be interested in identifying factors or covariates that are predictive of the outcome of interest. To model these type of count data we can use Poisson regression where the numbers are considered as counts rather than ranking. 
In medical and epidemiologic studies, data are often obtained in which the dependent variable is a count such as the number of cancer deaths, that is described by a set of predictor variables. A particular interest can occur in epidemiologic follow-up studies where data are organized into a format similar to that of a life table. One or more factors may affect the survival experience under the cohort study that could include categorical variables (e.g. race, sex) or grouped values of exposure variables (Frome, 1983). To deal with these situations, Poisson models can be used.

An example is discussed in Frome (1983) using the best dose-response data for human cancer that are obtained by Doll (1971) in a study of cigarette smoking in British physicians. These data can be used to illustrate the Poisson regression methods using both log-linear and nonlinear regression models. Another common approach is to categorize the data into two categories; (1): one of the eggs hatched or none did, (2): more ordered categories such as no eggs hatched, 1-2 eggs hatched, 3 or more eggs hatched. Thus, this approach leads a logistic regression model but it throws away real information and often gives lower power.

In this chapter, we introduce the response model and notation to define the generalized linear model for count data and describe the maximum likelihood method for estimating parameters in a log-linear model. We also present results from a simulation study, which was carried out to investigate the empirical properties of the maximum likelihood approach. 


\subsection{Model and Notation}

\subsubsection{Poisson Model for Count Data}

The Poisson distribution of a random variable $Y$ with parameter $\mu$ can be expressed by the probability density function

$$
\operatorname{Pr}\left(Y_{i}=y_{i}\right)=\frac{e^{-\mu} \mu^{y}}{y !}
$$

where $y=0,1, \ldots$ and $\mu>0$. The mean and variance of this distribution can be shown as

$$
E(Y)=\operatorname{var}(Y)=\mu
$$

Since the mean and variance are equal, any factor that affects one will also affect the other.

\section{Log-Linear Model}

Suppose that we have a sample of $n$ observations. Treating $y_{i}$ as a realization of independent Poisson random variable $Y_{i}$ which takes the values $1,2, \ldots, n$ with mean $\mu_{i}$, we can write

$$
\mu_{i}=\mathbf{x}_{i}^{t} \boldsymbol{\beta}
$$

where the expected count $\mu_{i}$ depends on a vector of explanatory variables $x_{i}$. There are some limitations of this model. The linear predictor on the right hand side of this Eq.(5.2) can assume any real value, whereas the Poisson mean on the left hand side has to be non-negative. To deal with this problem, we can consider logarithm of the mean on the left hand side, assuming that the transformed mean follows a linear model. It 
can be written as

$$
\log \left(\mu_{i}\right)=\mathbf{x}_{i}{ }^{t} \boldsymbol{\beta},
$$

where $\eta_{i}=\mathbf{x}_{i}{ }^{t} \boldsymbol{\beta}$ is considered as a generalized linear model with the log link.

\subsubsection{Maximum Likelihood Estimation}

For $n$ independent Poisson observations, the likelihood function is a product of probabilities given by Eq.(5.1). The likelihood function is

$$
L=\prod_{i=1}^{n} \frac{e^{-\mu_{i}} \mu_{i}^{y_{i}}}{y_{i} !},
$$

where $\mu_{i}$ depends on the covariates $x_{i}$ and a vector $\boldsymbol{\beta}$ of $p$ parameters. The maximum likelihood estimates are the values for $\boldsymbol{\beta}$ which maximize the likelihood function in Eq.(5.4). Taking logarithms on both sides of Eq.(5.4) and ignoring the constant term, we get the log-likelihood function

$$
\begin{aligned}
\log L(\boldsymbol{\beta})= & \sum_{i=1}^{n}\left\{y_{i} \log \left(\mu_{i}\right)-\mu_{i}\right\} \\
& =\sum_{i=1}^{n}\left\{y_{i} x_{i}{ }^{t} \boldsymbol{\beta}-\exp \left(\mathbf{x}_{i}{ }^{t} \boldsymbol{\beta}\right)\right\} .
\end{aligned}
$$

To obtain the score equation, we take the first derivative of Eq.(5.5) with respect to $\boldsymbol{\beta}$, which gives

$$
\frac{\partial \log L}{\partial \boldsymbol{\beta}}=\sum_{i=1}^{n}\left\{y_{i} x_{i}-x_{i} \mu_{i}\right\} .
$$

The maximum likelihood estimators of $\boldsymbol{\beta}$ are obtained by solving the ML estimating equations. It can be shown that the maximum likelihood estimates in log-linear Poisson 
models satisfy the estimating equations

$$
\mathbf{X}^{t} \mathbf{X}=\mathbf{X}^{t} \hat{\boldsymbol{\mu}}
$$

where $\mathbf{X}$ is the design matrix, $\mathbf{y}$ is the response vector, and $\hat{\boldsymbol{\mu}}$ is a vector of fitted values, which is obtained from the MLEs $\hat{\boldsymbol{\beta}}$ by exponentiating the linear predictor $\hat{\eta}_{i}=\mathbf{x}_{i}{ }^{t} \hat{\boldsymbol{\beta}}$.

In general, $\hat{\boldsymbol{\beta}}$ cannot be obtained in a closed form. Some numerical algorithm such as the iteratively reweighted least squares (IRWLS) or Newton-Raphson method can be used to solve the equation.

When the events of interest follow the Poisson distribution, the maximum likelihood estimates can be obtained by the IRWLS algorithm which is equivalent to using the method of scoring (Frome, 1983). Under regularity conditions, Poisson regression models include not only log-linear models but also quasilinear and intrinsically nonlinear models. The selection of an approach enables one to describe the relation between the dependent variable and the predictor variables through the regression model.

\subsection{Simulation Study}

To assess the performance of the maximum likelihood method, we ran sixteen sets of simulations under four scenarios using the Poisson regression model with missing covariates. In the first two scenarios, the estimates were studied under correctly specified MAR models and NMAR models respectively. In the second two scenarios, the estimates were studied under misspecified models for the missing data. We investigated the empirical properties of the ML estimators of $\hat{\boldsymbol{\beta}}$ which may be adequate to allow the 
inferential procedures under normal theory for moderate sample sizes.

We computed $95 \%$ confidence intervals for the regression coefficients as well as for the nuisance parameters for each of the sixteen sets of simulations to investigate if departures from normality are sufficiently severe to adversely affect normal-theory parametric inferences. If the normality assumption is satisfied, then $t=\left(\hat{\theta_{n}}-\theta\right) /$ s.e. $\left(\hat{\theta_{n}}\right)$ approximately follows a Student's $t_{n-p}$ distribution, where $n$ is the total number of observations in the data set and $p$ is the number of parameters in the model. Then the nominal level of the confidence interval for $\theta$ is $100(1-\alpha)$ as obtained from the confidence interval $\hat{\theta_{n}} \pm t_{n-p ; \alpha / 2}$ s.e. $\left(\hat{\theta_{n}}\right)$.

\subsubsection{Poisson Model for Simulation}

Consider a Poisson model with two covariates $x_{1}$ and $x_{2}$. The covariates $\left(x_{i 1}, x_{i 2}\right)$ for the $i$ th individual are assumed to be independent normal with mean $\boldsymbol{\mu}_{x}$ and covariance matrix $\Sigma_{x}$. For the $i$ th response variable $y_{i}$, assume

$$
y_{i} \mid x_{1 i}, x_{2 i} \sim \text { independent Poisson }\left(\mu_{i}\right), i=1,2 \ldots n
$$

and

$$
\theta_{i}=\log \left(\mu_{i}\right)=\mathbf{x}_{i}^{t} \boldsymbol{\beta}=\beta_{0}+\beta_{1} x_{i 1}+\beta_{2} x_{i 2} .
$$

In this setting, we have $\mu_{i}=\mu_{i}\left(\boldsymbol{\beta}, \mathbf{x}_{\mathbf{i}}\right)=E\left\{y_{i} \mid \mathbf{x}_{\mathbf{i}}, \boldsymbol{\beta}\right\}=\exp \left(\mathbf{x}_{i}{ }^{t} \boldsymbol{\beta}\right)$ and $\operatorname{var}\left\{y_{i} \mid \mathbf{x}_{\mathbf{i}}, \boldsymbol{\beta}\right\}=$ $\sigma_{i}^{2}\left(\boldsymbol{\beta}, \mathbf{x}_{\mathbf{i}}\right)=\exp \left(\mathbf{x}_{i}{ }^{t} \boldsymbol{\beta}\right)$. Here, the values of the covariate $x_{1}$ are completely observed $\left(v_{1 i}=0\right.$ for all $\left.i\right)$ and some values of $x_{2}$ are missing according to the missing data model 
For the Poisson regression, a series of 1000 data were generated from Poisson model (5.6) when the sample sizes are $n=100$ and 200. We generated the values of the covariates $\left(x_{i 1}, x_{i 2}\right)$ from a bivariate normal distribution with mean $\boldsymbol{\mu}_{x}=(0.5,0.5)^{t}$ and covariance matrix

$$
\Sigma_{x}=\left(\begin{array}{ll}
.5 & .1 \\
.1 & .5
\end{array}\right)
$$

In our notation, $\boldsymbol{\alpha}=\left(\mu_{x_{1}}, \mu_{x_{2}}, \sigma_{x_{1}}^{2}, \sigma_{x_{2}}^{2}, \sigma_{x_{1} x_{2}}\right)^{t}=(.5, .5, .5, .5,0.1)^{t}$. The regression coefficients were fixed at $\boldsymbol{\beta}=(0.5,0.5,1)^{t}$ and $\boldsymbol{\beta}=(0.5,1,0.5)^{t}$.

We obtain the ML estimates of the regression parameters $\boldsymbol{\beta}$ as well as the nuisance parameters $\boldsymbol{\alpha}$ and $\boldsymbol{\tau}$ by the iterative Newton-Raphson method described earlier.

\subsubsection{Results and Discussion for the Poisson Model}

In this section, we studied empirical biases and mean square errors of the ML estimators under four scenarios, where each scenario contains four sets of simulations conducted under the Poisson regression model with missing covariates. We also computed $95 \%$ confidence intervals and average lengths of the confidence intervals for the regression coefficients $\boldsymbol{\beta}$ as well as the nuisance parameters $\boldsymbol{\tau}$ of the missing data mechanism for different sample sizes.

When the missing data mechanism is MAR, for the two choices of $\boldsymbol{\beta}=(0.5,0.5,1)^{t}$ and $\boldsymbol{\beta}=(0.5,1,0.5)^{t}$, the data contained roughly $21.1 \%$ and $19 \%$ missing values, respectively. On the other hand, when the missing data mechanism is NMAR, for the two 
Table 5.1: Empirical biases and mean squared errors (MSEs) of maximum likelihood estimators in Poisson regression models with missing covariates for different sample sizes. Slope parameter $\beta_{1}=0.5$. Correctly specified MAR model is assumed for missing data.

\begin{tabular}{cccccc}
\hline & & \multicolumn{2}{c}{$\mathrm{n}=100$} & \multicolumn{2}{c}{$\mathrm{n}=200$} \\
\hline Parameter & True value & Bias & MSE & Bias & MSE \\
\hline$\beta_{0}$ & 0.5 & 0.01193 & 0.01145 & 0.01292 & 0.00493 \\
$\beta_{1}$ & 0.5 & -0.00201 & 0.00653 & 0.000845 & 0.00286 \\
$\beta_{2}$ & 1 & 0.00113 & 0.00596 & 0.00036 & 0.00272 \\
$\mu_{1}$ & 0.5 & 0.00366 & 0.00468 & 0.00032 & 0.00256 \\
$\mu_{2}$ & 0.5 & -0.01108 & 0.00546 & -0.01602 & 0.00298 \\
$\sigma_{1}^{2}$ & 0.5 & -0.00583 & 0.00506 & -0.00045 & 0.00243 \\
$\sigma_{2}^{2}$ & 0.5 & 0.00162 & 0.00554 & -0.00299 & 0.00293 \\
$\sigma_{12}$ & 0.1 & 0.00152 & 0.00271 & -0.00104 & 0.00144 \\
\hline
\end{tabular}

choices of $\boldsymbol{\beta}=(0.5,0.5,1)^{t}$ and $\boldsymbol{\beta}=(0.5,1,0.5)^{t}$, the data contained roughly $42.2 \%$ and $40.3 \%$ missing values, respectively. The simulation results are discussed here under four scenarios as presented bellow.

Scenario 1: True model: $\operatorname{MAR}\left(\tau_{3}=0\right)$; fitted model: $\operatorname{MAR}\left(\tau_{3}=0\right)$.

In this scenario, the data were generated by using the MAR model where the parameters of the missing-data mechanism were chosen as $\boldsymbol{\tau}=(-2,0.5,0.5,0)^{t}$ and the data were fitted by using the MAR model as well. In that sense, the model is correctly specified. The regression coefficients were fixed at $\boldsymbol{\beta}=(0.5,0.5,1)^{t}$ and $\boldsymbol{\beta}=(0.5,1,0.5)^{t}$. As the model is correctly specified, we expect to get negligible biases, small MSEs and good coverage probabilities for the estimators.

Table 5.1 presents the simulated biases and mean squared errors, and Table 5.2 
Table 5.2: Coverage probabilities (CPs), average lengths of maximum likelihood estimators in Poisson regression models with missing covariates for different sample sizes. Slope parameter $\beta_{1}=0.5$. Correctly specified MAR model is assumed for missing data.

\begin{tabular}{cccccc}
\hline & & \multicolumn{2}{c}{$\mathrm{n}=100$} & \multicolumn{2}{c}{$\mathrm{n}=200$} \\
\hline Parameter & True value & $\mathrm{CP}$ & Length & $\mathrm{CP}$ & Length \\
\hline$\beta_{0}$ & 0.5 & 0.93763 & 0.39242 & 0.94294 & 0.27354 \\
$\beta_{1}$ & 0.5 & 0.93763 & 0.30333 & 0.94895 & 0.20904 \\
$\beta_{2}$ & 1 & 0.95297 & 0.29974 & 0.95295 & 0.20760 \\
\hline
\end{tabular}

Table 5.3: Empirical biases and mean squared errors (MSEs) of maximum likelihood estimators in Poisson regression models with missing covariates for different sample sizes. Slope parameter $\beta_{1}=1$. Correctly specified MAR model is assumed for missing data.

\begin{tabular}{cccccc}
\hline & & \multicolumn{2}{c}{$\mathrm{n}=100$} & \multicolumn{2}{c}{$\mathrm{n}=200$} \\
\hline Parameter & True value & Bias & MSE & Bias & MSE \\
\hline$\beta_{0}$ & 0.5 & 0.00778 & 0.00901 & 0.00954 & 0.00453 \\
$\beta_{1}$ & 1 & 0.00292 & 0.00578 & 0.00074 & 0.00272 \\
$\beta_{2}$ & 0.5 & 0.00241 & 0.00593 & 0.00586 & 0.00267 \\
$\mu_{1}$ & 0.5 & -0.00111 & 0.00557 & -0.00191 & 0.00239 \\
$\mu_{2}$ & 0.5 & -0.02698 & 0.00608 & -0.03061 & 0.00367 \\
$\sigma_{1}^{2}$ & 0.5 & -0.00326 & 0.00473 & -0.00149 & 0.00256 \\
$\sigma_{2}^{2}$ & 0.5 & -0.00950 & 0.00609 & -0.00851 & 0.00299 \\
$\sigma_{12}$ & 0.1 & -0.00321 & 0.00274 & -0.00206 & 0.00145 \\
\hline
\end{tabular}


Table 5.4: Coverage probabilities (CPs), average lengths of maximum likelihood estimators in Poisson regression models with missing covariates for different sample sizes. Slope parameter $\beta_{1}=1$. Correctly specified MAR model is assumed for missing data.

\begin{tabular}{cccccc}
\hline & & \multicolumn{2}{c}{$\mathrm{n}=100$} & \multicolumn{2}{c}{$\mathrm{n}=200$} \\
\hline Parameter & True value & $\mathrm{CP}$ & Length & $\mathrm{CP}$ & Length \\
\hline$\beta_{0}$ & 0.5 & 0.95282 & 0.38143 & 0.94394 & 0.26615 \\
$\beta_{1}$ & 1 & 0.94974 & 0.29508 & 0.94995 & 0.20328 \\
$\beta_{2}$ & 0.5 & 0.94667 & 0.29112 & 0.94995 & 0.20126 \\
\hline
\end{tabular}

presents the coverage probabilities and the mean lengths of the confidence intervals for different sample sizes, where the regression coefficients were fixed at $\boldsymbol{\beta}=(0.5,0.5,1)^{t}$ and the parameters of the missing-data mechanism were chosen as $\boldsymbol{\tau}=(-2,0.5,0.5,0)^{t}$.

On the other hand, to study the impact of changes to the coefficients of missing covariates, we consider the regression coefficients $\boldsymbol{\beta}=(0.5,1,0.5)^{t}$ keeping the nuisance parameters $\boldsymbol{\alpha}$ of the covariate distribution and $\boldsymbol{\tau}$ of the missing data mechanism similar to those as used for correctly specified models under the MAR setting.

Table 5.3 presents the simulated biases and mean squared errors, and Table 5.4 presents the coverage probabilities and mean lengths of the confidence intervals for different sample sizes under the same setting as above but the regression coefficients were fixed at $\boldsymbol{\beta}=(0.5,1,0.5)^{t}$.

The maximum likelihood method appears to perform well for the correctly specified MAR mechanism under different sample sizes. The method provides unbiased estima- 
tors and small mean squared errors for all the regression coefficients $\boldsymbol{\beta}$ as well as the nuisance parameters $\boldsymbol{\alpha}$ of the covariate distribution in both Tables 5.1 and 5.3. The confidence intervals have good coverages, which are close to the nominal $95 \%$ confidence level.

As expected, the biases and mean squared errors for all the regression coefficients $\boldsymbol{\beta}$ as well as the nuisance parameters $\boldsymbol{\alpha}$ become smaller when we increase the sample size. The coverage probabilities for the parameter estimates become closer to the nominal levels and the mean lengths based on confidence interval become smaller when we increase the sample size. The confidence intervals for the regression coefficients $\boldsymbol{\beta}$ slightly improve coverages due to the choice of different values of $\boldsymbol{\beta}=(0.5,1,0.5)^{t}$. As shown in Table 5.4, $\beta_{1}$ improves its coverage to 0.94974 from 0.93763 when the sample size is $n=100$.

Scenario 2: True model: $\operatorname{NMAR}\left(\tau_{3}=0.25\right)$; fitted model: $\operatorname{NMAR}\left(\tau_{3} \neq 0\right)$. In this scenario, the data were generated by using the NMAR model where the parameters of the missing-data mechanism were chosen as $\boldsymbol{\tau}=(-2,0.5,0.5,0.25)^{t}$ and the data were fitted by using the NMAR model as well. In this case, the model is correctly specified. The regression coefficients were fixed at $\boldsymbol{\beta}=(0.5,0.5,1)^{t}$ and $\boldsymbol{\beta}=(0.5,1,0.5)^{t}$. Here, we expect to get unbiased and efficient estimators.

Table 5.5 presents the simulated biases and mean squared errors, and Table 5.6 presents the coverage probabilities and the mean lengths of the confidence intervals for different sample sizes, where the regression coefficients were fixed at $\boldsymbol{\beta}=(0.5,0.5,1)^{t}$ 
Table 5.5: Empirical biases and mean squared errors (MSEs) of maximum likelihood estimators in Poisson regression models with missing covariates for different sample sizes. Slope parameter $\beta_{1}=0.5$. Correctly specified NMAR model is assumed for missing data.

\begin{tabular}{cccccc}
\hline & & \multicolumn{2}{c}{$\mathrm{n}=100$} & \multicolumn{2}{c}{$\mathrm{n}=200$} \\
\cline { 2 - 5 } Parameter & True value & Bias & MSE & Bias & MSE \\
\hline$\beta_{0}$ & 0.5 & 0.00267 & 0.01770 & 0.00341 & 0.00847 \\
$\beta_{1}$ & 0.5 & 0.00568 & 0.01034 & 0.00605 & 0.00505 \\
$\beta_{2}$ & 1 & 0.00033 & 0.01108 & $6.65815 \mathrm{E}-05$ & 0.00503 \\
$\tau_{0}$ & -2 & -0.38703 & 0.66805 & -0.16094 & 0.17269 \\
$\tau_{1}$ & 0.5 & 0.05145 & 0.39414 & -0.01083 & 0.14341 \\
$\tau_{2}$ & 0.5 & -0.04150 & 2.09604 & -0.08365 & 0.86136 \\
$\tau_{3}$ & 0.25 & 0.05866 & 0.06127 & 0.03447 & 0.02397 \\
$\mu_{1}$ & 0.5 & -0.00192 & 0.00476 & 0.00032 & 0.00252 \\
$\mu_{2}$ & 0.5 & -0.01166 & 0.00887 & -0.00899 & 0.00423 \\
$\sigma_{1}^{2}$ & 0.5 & -0.00419 & 0.00544 & 0.00165 & 0.00245 \\
$\sigma_{2}^{2}$ & 0.5 & 0.00422 & 0.00800 & 0.00391 & 0.00356 \\
$\sigma_{12}$ & 0.1 & -0.00378 & 0.00328 & -0.00040 & 0.00168 \\
\hline
\end{tabular}


Table 5.6: Coverage probabilities (CPs), average lengths of maximum likelihood estimators in Poisson regression models with missing covariates for different sample sizes. Slope parameter $\beta_{1}=0.5$. Correctly specified NMAR model is assumed for missing data.

\begin{tabular}{cccccc}
\hline & & \multicolumn{2}{c}{$\mathrm{n}=100$} & \multicolumn{2}{c}{$\mathrm{n}=200$} \\
\hline Parameter & True value & $\mathrm{CP}$ & Length & $\mathrm{CP}$ & Length \\
\hline$\beta_{0}$ & 0.5 & 0.88648 & 0.43370 & 0.90010 & 0.30510 \\
$\beta_{1}$ & 0.5 & 0.91847 & 0.37311 & 0.93170 & 0.26211 \\
$\beta_{2}$ & 1 & 0.92260 & 0.36548 & 0.94190 & 0.25669 \\
$\tau_{0}$ & -2 & 0.96594 & 2.31066 & 0.94801 & 1.40205 \\
$\tau_{1}$ & 0.5 & 0.93086 & 1.98681 & 0.92966 & 1.31357 \\
$\tau_{2}$ & 0.5 & 0.86068 & 3.93896 & 0.85831 & 2.61866 \\
$\tau_{3}$ & 0.25 & 0.89164 & 0.66541 & 0.87462 & 0.44538 \\
\hline
\end{tabular}

and the parameters of the missing-data model were chosen as $\boldsymbol{\tau}=(-2,0.5,0.5,0.25)^{t}$.

On the other hand, to study the impact of changes to the coefficients of missing covariates, we consider the regression coefficients $\boldsymbol{\beta}=(0.5,1,0.5)^{t}$ keeping the nuisance parameters $\boldsymbol{\alpha}$ of the covariate distribution and $\boldsymbol{\tau}$ of the missing data mechanism similar to those as used for correctly specified models under the NMAR setting.

Table 5.7 presents the simulated biases and mean squared errors, and Table 5.8 presents the coverage probabilities and the mean lengths of the confidence intervals for different sample sizes under the same setting but the regression coefficients were fixed at $\boldsymbol{\beta}=(0.5,1,0.5)^{t}$.

The maximum likelihood method appears to perform well for the correctly specified 
Table 5.7: Empirical biases and mean squared errors (MSEs) of maximum likelihood estimators in Poisson regression models with missing covariates for different sample sizes. Slope parameter $\beta_{1}=1$. Correctly specified NMAR model is assumed for missing data.

\begin{tabular}{cccccc}
\hline & & \multicolumn{2}{c}{$\mathrm{n}=100$} & \multicolumn{2}{c}{$\mathrm{n}=200$} \\
\cline { 2 - 5 } Parameter & True value & Bias & MSE & Bias & MSE \\
\hline$\beta_{0}$ & 0.5 & 0.00500 & 0.01225 & 0.00439 & 0.00660 \\
$\beta_{1}$ & 1 & -0.000890 & 0.00870 & 0.00260 & 0.00428 \\
$\beta_{2}$ & 0.5 & -0.00533 & 0.00831 & -0.00333 & 0.00379 \\
$\tau_{0}$ & -2 & -0.29387 & 0.48109 & -0.13770 & 0.17931 \\
$\tau_{1}$ & 0.5 & 0.05645 & 0.58071 & -0.00401 & 0.25321 \\
$\tau_{2}$ & 0.5 & -0.00776 & 0.94030 & -0.03022 & 0.45248 \\
$\tau_{3}$ & 0.25 & 0.04216 & 0.03283 & 0.02315 & 0.01399 \\
$\mu_{1}$ & 0.5 & -0.00112 & 0.00512 & 0.00129 & 0.00245 \\
$\mu_{2}$ & 0.5 & -0.01241 & 0.01162 & -0.00565 & 0.00566 \\
$\sigma_{1}^{2}$ & 0.5 & -0.00071 & 0.00490 & 0.00083 & 0.00228 \\
$\sigma_{2}^{2}$ & 0.5 & 0.00109 & 0.00856 & 0.00321 & 0.00409 \\
$\sigma_{12}$ & 0.1 & 0.00039 & 0.00420 & -0.00285 & 0.02531 \\
\hline
\end{tabular}

Table 5.8: Coverage probabilities (CPs), average lengths of maximum likelihood estimators in Poisson regression models with missing covariates for different sample sizes. Slope parameter $\beta_{1}=1$. Correctly specified NMAR model is assumed for missing data.

\begin{tabular}{cccccc}
\hline & & \multicolumn{2}{c}{$\mathrm{n}=100$} & \multicolumn{2}{c}{$\mathrm{n}=200$} \\
\cline { 2 - 5 } Parameter & True value & $\mathrm{CP}$ & Length & $\mathrm{CP}$ & Length \\
\hline$\beta_{0}$ & 0.5 & 0.91203 & 0.40589 & 0.92764 & 0.28741 \\
$\beta_{1}$ & 1 & 0.92518 & 0.33497 & 0.93266 & 0.23707 \\
$\beta_{2}$ & 0.5 & 0.92720 & 0.31939 & 0.92965 & 0.22441 \\
$\tau_{0}$ & -2 & 0.96967 & 2.22625 & 0.95378 & 1.41763 \\
$\tau_{1}$ & 0.5 & 0.94034 & 2.59135 & 0.92663 & 1.75404 \\
$\tau_{2}$ & 0.5 & 0.90495 & 2.85656 & 0.86231 & 1.90343 \\
$\tau_{3}$ & 0.25 & 0.92417 & 0.56316 & 0.90854 & 0.37863 \\
\hline
\end{tabular}


model under the NMAR mechanism in terms of bias and MSE for different sample sizes. The method provides unbiased estimators and small mean squared errors for all the regression coefficients $\boldsymbol{\beta}$ as well as the nuisance parameters $\boldsymbol{\alpha}$ of the covariate distribution and $\boldsymbol{\tau}$ of the missing data mechanism. But $\tau_{0}$ shows slightly large biases in both Tables 5.5 and 5.7. The confidence intervals have coverages which are not very close to the nominal level. The estimates of nuisance parameter $\boldsymbol{\tau}$ slightly improve coverages due to the choice of different values of $\boldsymbol{\beta}=(0.5,1,0.5)^{t}$. As shown in Table $5.8, \tau_{2}$ improves its coverage to 0.90495 from 0.86068 when the sample size is $n=100$.

As expected, the biases and mean squared errors for all the regression coefficients $\boldsymbol{\beta}$ as well as the nuisance parameters $\boldsymbol{\alpha}$ and $\boldsymbol{\tau}$ become smaller when we increase the sample size. The coverage probabilities for the parameter estimates of $\boldsymbol{\beta}$ tend to be closer to the nominal level and the mean lengths based on confidence interval become smaller when we increase the sample size. Although we expected good coverages for the confidence intervals of nuisance parameters $\tau$ but it loses slight coverage for both choices of $\boldsymbol{\beta}=(-2,1,0.5)^{t}$ and $\boldsymbol{\beta}=(-2,0.5,1)^{t}$ as shown in both Tables 5.6 and 5.8 when we increase the sample size.

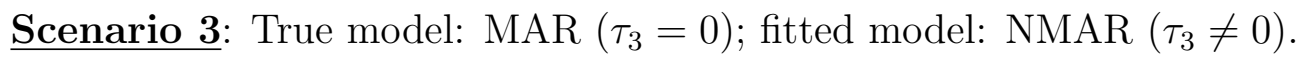
In this scenario, the data were generated by using the MAR model where the parameters of the missing-data mechanism were chosen as $\boldsymbol{\tau}=(-2,0.5,0.5,0)^{t}$ and the data were fitted by using the NMAR model as well. In this case, the model is misspecified. The regression coefficients were fixed at $\boldsymbol{\beta}=(0.5,0.5,1)^{t}$ and $\boldsymbol{\beta}=(0.5,1,0.5)^{t}$. Here, we expect to get larger biases, MSEs and bad coverages for the estimators under the 
Table 5.9: Empirical biases and mean squared errors (MSEs) of maximum likelihood estimators in Poisson regression models with missing covariates for different sample sizes. Slope parameter $\beta_{1}=0.5$. Misspecified NMAR model is assumed for missing data.

\begin{tabular}{cccccc}
\hline & & \multicolumn{2}{c}{$\mathrm{n}=100$} & \multicolumn{2}{c}{$\mathrm{n}=200$} \\
\hline Parameter & True value & Bias & MSE & Bias & MSE \\
\hline$\beta_{0}$ & 0.5 & -0.01081 & 0.01258 & -0.00138 & 0.00515 \\
$\beta_{1}$ & 0.5 & 0.00613 & 0.00652 & -0.000468 & 0.00288 \\
$\beta_{2}$ & 1 & -0.00303 & 0.00683 & -0.00012 & 0.00266 \\
$\tau_{0}$ & -2 & -0.41179 & 1.11462 & -0.16063 & 0.21130 \\
$\tau_{1}$ & 0.5 & 0.15571 & 0.45363 & 0.06695 & 0.13993 \\
$\tau_{2}$ & 0.5 & 0.29816 & 3.14971 & 0.12177 & 0.89027 \\
$\tau_{3}$ & 0 & -0.02528 & 0.04094 & -0.01057 & 0.011035 \\
$\mu_{1}$ & 0.5 & -0.00063 & 0.00491 & 0.00306 & 0.00243 \\
$\mu_{2}$ & 0.5 & 0.00221 & 0.00706 & 0.00245 & 0.00343 \\
$\sigma_{1}^{2}$ & 0.5 & -0.00010 & 0.00502 & -0.00110 & 0.00240 \\
$\sigma_{2}^{2}$ & 0.5 & 0.00995 & 0.00589 & 0.00202 & 0.00285 \\
$\sigma_{12}$ & 0.1 & 0.00280 & 0.00305 & -0.00173 & 0.00134 \\
\hline
\end{tabular}

misspecified model as compared to the correctly specified models.

Table 5.9 presents the simulated biases and mean squared errors, and Table 5.10 presents the coverage probabilities and the mean lengths of the confidence intervals for different sample sizes, where the regression coefficients were fixed at $\boldsymbol{\beta}=(0.5,0.5,1)^{t}$ and the parameters of the missing-data mechanism were chosen as $\boldsymbol{\tau}=(-2,0.5,0.5,0)^{t}$.

On the other hand, to study the impact of changes to the coefficients of missing covariates, we consider the regression coefficients $\boldsymbol{\beta}=(0.5,1,0.5)^{t}$ keeping the nuisance parameters $\boldsymbol{\alpha}$ of the covariate distribution and $\boldsymbol{\tau}$ of the missing data mechanism similar 
Table 5.10: Coverage probabilities (CPs), average lengths of maximum likelihood estimators in Poisson regression models with missing covariates for different sample sizes. Slope parameter $\beta_{1}=0.5$. Misspecified NMAR model is assumed for missing data.

\begin{tabular}{cccccc}
\hline & & \multicolumn{2}{c}{$\mathrm{n}=100$} & \multicolumn{2}{c}{$\mathrm{n}=200$} \\
\cline { 2 - 6 } Parameter & True value & $\mathrm{CP}$ & Length & $\mathrm{CP}$ & Length \\
\hline$\beta_{0}$ & 0.5 & 0.91174 & 0.38585 & 0.93494 & 0.26996 \\
$\beta_{1}$ & 0.5 & 0.91976 & 0.28920 & 0.93193 & 0.20296 \\
$\beta_{2}$ & 1 & 0.91575 & 0.28957 & 0.94695 & 0.20043 \\
$\tau_{0}$ & -2 & 0.97192 & 2.59766 & 0.96697 & 1.48941 \\
$\tau_{1}$ & 0.5 & 0.93882 & 2.12699 & 0.95596 & 1.36049 \\
$\tau_{2}$ & 0.5 & 0.87162 & 4.38257 & 0.89489 & 2.74803 \\
$\tau_{3}$ & 0 & 0.89569 & 0.49687 & 0.90891 & 0.29726 \\
\hline
\end{tabular}

Table 5.11: Empirical biases and mean squared errors (MSEs) of maximum likelihood estimators in Poisson regression models with missing covariates for different sample sizes. Slope parameter $\beta_{1}=1$. Misspecified NMAR model is assumed for missing data.

\begin{tabular}{cccccc}
\hline & & \multicolumn{2}{c}{$\mathrm{n}=100$} & \multicolumn{2}{c}{$\mathrm{n}=200$} \\
\cline { 2 - 5 } Parameter & True value & Bias & MSE & Bias & MSE \\
\hline$\beta_{0}$ & 0.5 & -0.00595 & 0.01140 & -0.00247 & 0.00542 \\
$\beta_{1}$ & 1 & 0.00649 & 0.00625 & 0.00513 & 0.00278 \\
$\beta_{2}$ & 0.5 & -0.00879 & 0.00636 & -0.00635 & 0.00279 \\
$\tau_{0}$ & -2 & -0.51473 & 1.45177 & -0.21195 & 0.36304 \\
$\tau_{1}$ & 0.5 & 0.24751 & 1.09658 & 0.10283 & 0.37407 \\
$\tau_{2}$ & 0.5 & 0.28992 & 2.14289 & 0.08173 & 0.80420 \\
$\tau_{3}$ & 0 & -0.02978 & 0.03239 & -0.00926 & 0.01053 \\
$\mu_{1}$ & 0.5 & 0.00104 & 0.00528 & 0.00029 & 0.00229 \\
$\mu_{2}$ & 0.5 & 0.00617 & 0.00851 & -0.00144 & 0.00468 \\
$\sigma_{1}^{2}$ & 0.5 & -0.00276 & 0.00486 & -0.00096 & 0.00249 \\
$\sigma_{2}^{2}$ & 0.5 & 0.02336 & 0.00707 & 0.01116 & 0.00336 \\
$\sigma_{12}$ & 0.1 & 0.00066 & 0.00332 & $8.65232 \mathrm{E}-07$ & 0.00148 \\
\hline
\end{tabular}


Table 5.12: Coverage probabilities (CPs), average lengths of maximum likelihood estimators in Poisson regression models with missing covariates for different sample sizes. Slope parameter $\beta_{1}=1$. Misspecified NMAR model is assumed for missing data.

\begin{tabular}{cccccc}
\hline & & \multicolumn{2}{c}{$\mathrm{n}=100$} & \multicolumn{2}{c}{$\mathrm{n}=200$} \\
\hline Parameter & True value & $\mathrm{CP}$ & Length & $\mathrm{CP}$ & Length \\
\hline$\beta_{0}$ & 0.5 & 0.91592 & 0.37541 & 0.925 & 0.26227 \\
$\beta_{1}$ & 1 & 0.90591 & 0.27683 & 0.928 & 0.19482 \\
$\beta_{2}$ & 0.5 & 0.90591 & 0.27235 & 0.924 & 0.19259 \\
$\tau_{0}$ & -2 & 0.96797 & 2.88646 & 0.945 & 1.62977 \\
$\tau_{1}$ & 0.5 & 0.93594 & 2.94996 & 0.925 & 1.82153 \\
$\tau_{2}$ & 0.5 & 0.86386 & 3.56772 & 0.808 & 2.22634 \\
$\tau_{3}$ & 0 & 0.91291 & 0.44219 & 0.889 & 0.26171 \\
\hline
\end{tabular}

to those as used for misspecified models under the same setting.

Table 5.11 presents the simulated biases and mean squared errors, and Table 5.12 presents the coverage probabilities and the mean lengths of the confidence intervals for different sample sizes under same setting, but the regression coefficients were fixed at $\boldsymbol{\beta}=(0.5,1,0.5)^{t}$.

The maximum likelihood method doesn't appear to perform well for this misspecified model in terms of coverage probability for different sample sizes. The method still provides small biases and mean squared errors for all the regression coefficients $\boldsymbol{\beta}$ as well as the nuisance parameters $\boldsymbol{\alpha}$ of the covariate distribution in both Tables 5.9 and 5.11 . But the method produces larger biases and mean squared errors for $\boldsymbol{\tau}$ of the missing data mechanism compared to Scenario 2 for both choices of $\boldsymbol{\beta}=(0.5,0.5,1)^{t}$ 
and $\boldsymbol{\beta}=(0.5,1,0.5)^{t}$. The parameter estimates have bad coverages that moved away from their nominal levels. The confidence intervals for the nuisance parameter $\boldsymbol{\tau}$ lose coverages due to the choice of different values of $\boldsymbol{\beta}=(0.5,1,0.5)^{t}$. As shown in Table $5.12, \tau_{2}$ loses its coverage to 0.808 from 0.89489 when the sample size is $n=200$.

Based on our simulation, the biases and mean squared errors for all the regression coefficients $\boldsymbol{\beta}$ as well as the nuisance parameters $\boldsymbol{\alpha}$ become smaller when we increase sample size. Although biases are large for $\boldsymbol{\tau}$ with $\mathrm{n}=100$, they become smaller when we increase the sample size. The coverage probabilities for the parameter estimates $\boldsymbol{\beta}$ become close to their nominal levels and the coverage probabilities for the parameter estimates $\boldsymbol{\tau}$ moved away from their nominal levels when we increase the sample size. Also, the mean lengths based on confidence interval become smaller with the large sample size.

Scenario 4: True model: NMAR $\left(\tau_{3}=0.25\right)$; fitted model: $\operatorname{MAR}\left(\tau_{3}=0\right)$. In this scenario, the data were generated by using the NMAR model where the parameters of the missing-data mechanism were chosen as $\tau=(-2,0.5,0.5,0.25)^{t}$ and the data were fitted by using the MAR model. In this case, the model is misspecified. The regression coefficients were fixed at $\boldsymbol{\beta}=(0.5,0.5,1)^{t}$ and $\boldsymbol{\beta}=(0.5,1,0.5)^{t}$.

Table 5.13 presents the simulated biases and mean squared errors, and Table 5.14 presents the coverage probabilities and the mean lengths of the confidence intervals for different sample sizes, where the regression coefficients were fixed at $\boldsymbol{\beta}=(0.5,0.5,1)^{t}$ and the parameters of the missing data mechanism were chosen as $\boldsymbol{\tau}=(-2,0.5,0.5,0.25)^{t}$. 
Table 5.13: Empirical biases and mean squared errors (MSEs) of maximum likelihood estimators in Poisson regression models with missing covariates for different sample sizes. Slope parameter $\beta_{1}=0.5$. Misspecified MAR model is assumed for missing data.

\begin{tabular}{cccccc}
\hline & & \multicolumn{2}{c}{$\mathrm{n}=100$} & \multicolumn{2}{c}{$\mathrm{n}=200$} \\
\hline Parameter & True value & Bias & MSE & Bias & MSE \\
\hline$\beta_{0}$ & 0.5 & 0.01645 & 0.014816 & 0.01686 & 0.00698 \\
$\beta_{1}$ & 0.5 & 0.00621 & 0.01259 & 0.014430 & 0.00575 \\
$\beta_{2}$ & 1 & 0.01576 & 0.01373 & 0.01030 & 0.00581 \\
$\mu_{1}$ & 0.5 & -0.00378 & 0.00527 & -0.00035 & 0.00231 \\
$\mu_{2}$ & 0.5 & -0.03423 & 0.00752 & -0.02947 & 0.00384 \\
$\sigma_{1}^{2}$ & 0.5 & -0.00353 & 0.00487 & -0.00316 & 0.00244 \\
$\sigma_{2}^{2}$ & 0.5 & -0.01272 & 0.00741 & -0.01565 & 0.00408 \\
$\sigma_{12}$ & 0.1 & -0.00603 & 0.00315 & -0.00556 & 0.00170 \\
\hline
\end{tabular}

Table 5.14: Coverage probabilities (CPs), average lengths of maximum likelihood estimators in Poisson regression models with missing covariates for different sample sizes. Slope parameter $\beta_{1}=0.5$. Misspecified MAR model is assumed for missing data.

\begin{tabular}{cccccc}
\hline & & \multicolumn{2}{c}{$\mathrm{n}=100$} & \multicolumn{2}{c}{$\mathrm{n}=200$} \\
\hline Parameter & True value & $\mathrm{CP}$ & Length & $\mathrm{CP}$ & Length \\
\hline$\beta_{0}$ & 0.5 & 0.92612 & 0.44654 & 0.94194 & 0.31297 \\
$\beta_{1}$ & 0.5 & 0.93653 & 0.41384 & 0.93894 & 0.28798 \\
$\beta_{2}$ & 1 & 0.92092 & 0.41008 & 0.93894 & 0.28403 \\
\hline
\end{tabular}


Table 5.15: Empirical biases and mean squared errors (MSEs) of maximum likelihood estimators in Poisson regression models with missing covariates for different sample sizes. Slope parameter $\beta_{1}=1$. Misspecified MAR model is assumed for missing data.

\begin{tabular}{cccccc}
\hline & & \multicolumn{2}{c}{$\mathrm{n}=100$} & \multicolumn{2}{c}{$\mathrm{n}=200$} \\
\hline Parameter & True value & Bias & MSE & Bias & MSE \\
\hline$\beta_{0}$ & 0.5 & 0.02366 & 0.01247 & 0.02035 & 0.00583 \\
$\beta_{1}$ & 1 & 0.02202 & 0.010450 & 0.01687 & 0.00530 \\
$\beta_{2}$ & 0.5 & -0.00454 & 0.01114 & 0.00262 & 0.00362 \\
$\mu_{1}$ & 0.5 & -0.00018 & 0.00524 & 0.00031 & 0.00238 \\
$\mu_{2}$ & 0.5 & -0.06373 & 0.01248 & -0.06281 & 0.00771 \\
$\sigma_{1}^{2}$ & 0.5 & 0.00038 & 0.00516 & -0.00310 & 0.00226 \\
$\sigma_{2}^{2}$ & 0.5 & -0.02717 & 0.00845 & -0.02487 & 0.00427 \\
$\sigma_{12}$ & 0.1 & -0.02227 & 0.00452 & -0.01601 & 0.00236 \\
\hline
\end{tabular}

Table 5.16: Coverage probabilities (CPs), average lengths of maximum likelihood estimators in Poisson regression models with missing covariates for different sample sizes. Slope parameter $\beta_{1}=1$. Misspecified MAR model is assumed for missing data.

\begin{tabular}{cccccc}
\hline & & \multicolumn{2}{c}{$\mathrm{n}=100$} & \multicolumn{2}{c}{$\mathrm{n}=200$} \\
\hline Parameter & True value & $\mathrm{CP}$ & Length & $\mathrm{CP}$ & Length \\
\hline$\beta_{0}$ & 0.5 & 0.93569 & 0.41527 & 0.93594 & 0.29119 \\
$\beta_{1}$ & 1 & 0.93891 & 0.38320 & 0.93594 & 0.26961 \\
$\beta_{2}$ & 0.5 & 0.94427 & 0.34806 & 0.95996 & 0.24165 \\
\hline
\end{tabular}


On the other hand, to study the impact of changes to the coefficients of missing covariates, we consider the regression coefficients $\boldsymbol{\beta}=(0.5,1,0.5)^{t}$ keeping the nuisance parameters $\boldsymbol{\alpha}$ of the covariate distribution and $\boldsymbol{\tau}$ of the missing data mechanism similar to those as used for misspecified models under the same setting.

Table 5.15 presents the simulated biases and mean squared errors, and Table 5.16 presents the coverage probabilities and the mean lengths of the parameter estimates for different sample sizes under same setting, but the regression coefficients were fixed at $\boldsymbol{\beta}=(0.5,1,0.5)^{t}$

The maximum likelihood method still performs well under this misspecified model for different sample sizes. The method provides small biases and mean squared errors for all the regression coefficients $\boldsymbol{\beta}$ as well as the nuisance parameters $\boldsymbol{\alpha}$ of the covariate distribution of the missing data mechanism in both Tables 5.13 and 5.15. The parameter estimates $\boldsymbol{\beta}$ lose slight coverages due to the choice of $\boldsymbol{\beta}=(0.5,0.5,1)^{t}$ in Table 5.14 but still they are close to their nominal levels $95 \%$.

Based on our simulation, the biases and mean squared errors for all the regression coefficients $\boldsymbol{\beta}$ as well as the nuisance parameters $\boldsymbol{\alpha}$ stay very close when we increase the sample size. The coverage probabilities for the parameter estimates slightly moved away from the nominal level but still the parameter estimates have the good coverages. The mean lengths based on confidence intervals become smaller and coverage probabilities stay very close when we increase the sample size. As seen, the CPs of $\beta_{1}$ are 0.93653 and 0.93894 in Table 5.14 as well as 0.93891 and 0.93594 in Table 5.16 when the sample 
sizes are $n=100$ and $n=200$ respectively.

It is clear from the above four scenarios that Scenario 1, 2 and 4 provide approximately unbiased estimates for all regression coefficients $\boldsymbol{\beta}$ as well as the nuisance parameters $\boldsymbol{\alpha}$ of the covariate distribution and $\boldsymbol{\tau}$ of the missing data mechanism under all simulation configurations considered. On the other hand, Scenario 3 provides approximately unbiased estimates of the regression coefficients $\boldsymbol{\beta}$ as well as the nuisance parameters $\boldsymbol{\alpha}$ but slightly large biases for the estimates of nuisance parameters $\boldsymbol{\tau}$. As shown in Table 5.9, under misspecified method, the estimators of $\tau_{1}$ and $\tau_{2}$ give large biases as 0.15571 and 0.29816 respectively. Also as shown in Table 5.11, 0.24751 and 0.28992 respectively when the sample size is $n=100$.

When comparing four scenarios, the maximum likelihood method performs better for Scenario 1, which is correctly specified model under the MAR mechanism and worst for Scenario 3, which is misspecified model under NMAR mechanism. As shown in Tables 5.1 and 5.3, Scenario 1 provides approximately unbiased estimates. Also, Scenario 2, 3 and 4 provide quite small biases and MSEs for all the parameter estimates of $\boldsymbol{\beta}$ and $\boldsymbol{\alpha}$ under both correctly specified and misspecified models for both choices of $\boldsymbol{\beta}=(0.5,0.5,1)^{t}$ and $\boldsymbol{\beta}=(0.5,1,0.5)^{t}$.

Scenario 1 also gives the better coverage probabilities and mean lengths for the regression coefficients $\boldsymbol{\beta}$ as shown in Table 5.2 and 5.4 under the correctly specified MAR mechanism. As we expected, the coverage probabilities are very close to the nominal level $95 \%$ which gradually improve and the mean lengths gradually decrease when we 
increase the sample size for both choices of $\boldsymbol{\beta}=(0.5,0.5,1)^{t}$ and $\boldsymbol{\beta}=(0.5,1,0.5)^{t}$. Scenario 2 and 3 give bad coverages for all parameter estimates of $\boldsymbol{\tau}$ and lose slight coverages when we increase the sample size to $n=200$ from $n=100$ due to the choice of $\boldsymbol{\beta}=(0.5,1,0.5)^{t}$. The coverages of $\boldsymbol{\tau}$ decrease for both Scenarios 2 and 3 accordingly with increased sample sizes.

In Scenario 4, the parameter estimates of $\boldsymbol{\beta}$ have good coverages and they stay close due to an increased sample sizes in both Tables 5.14 and 5.16, but they lose slight coverages for the choice of $\boldsymbol{\beta}=(0.5,0.5,1)^{t}$. For example, as in Table 5.14, $\beta_{2}$ gives empirical coverage probabilities to 0.92092 from 0.94427 when the sample size is $n=100$.

Here, we should note that there is no big bias for all estimates under the four scenarios. For the MSEs and mean lengths of the estimators of model parameters with different sample sizes, the empirical study shows that the MSEs and mean lengths decrease as the number of sample size $n$ increases. In terms of CPs, Scenarios 1 and 4 give good coverages under both specified and misspecified models. 


\section{Chapter 6}

\section{Conclusion}

In this thesis, we have studied the generalized linear model with missing covariates for analyzing binary and count data. We particularly considered joint estimation of the regression parameters and the association parameters by using the maximum likelihood method. We studied a set of maximum likelihood estimating equations for fitting regression models to binary data as well as Poisson data. We used the Newton-Raphson algorithm to estimate the parameters for both regressions. Our simulation study demonstrates that the missing data mechanism generally provides unbiased and efficient estimators when it follows correctly specified models under the NMAR method for binary data and MAR method for count data.

The purpose of the thesis was to study the empirical properties of the maximum likelihood estimator for assessing the significance of regression parameter $\boldsymbol{\beta}$ at a given level of significance. We also study the biases, MSEs, average lengths and empirical coverage probabilities of the estimators of model parameters under both correctly specified and misspecified models, when the true distribution is either the binomial or Poisson distribution. 
It is apparent that when we use the NMAR model to fit the data, the models provide unbiased and efficient estimators for binary regression. On the other hand, when we use the MAR model to fit the data, the models provide unbiased and efficient estimators for the Poisson regression.

From the numerical study, we conclude that when analyzing incomplete data with missing covariates, it is necessary to incorporate a suitable missing data model into the observed data likelihood function in order to obtain unbiased and efficient estimators of the model parameters. We also note that a misspecified missing data model can provide systematic bias in the maximum likelihood estimation. So it is important to assess the validity of a missing data model when performing a likelihood inference based on the given observed data.

\subsection{Future Research}

There are many current and future research considerations for missing data problems when the mechanism is nonignorable. In this thesis, we used univariate missing covariate, where only one covariate was considered missing. Analysis with multivariate missing covariates is computationally extensive. To reduce the computational burden, as a future research we plan to use an approximate method based on Markov Chain Monte Carlo approximation for fitting generalized linear models under nonignorable multivariate missing covariates. 


\section{Bibliography}

[1] Baker, S. G. and Laird, N. M. (1988). Regression analysis for categorical variables with outcome subject to nonresponse. Journal of the American Statistical Association, 83, 62-49.

[2] Barndorff Nielsen, O. (1983). On a formula for the distribution of the maximum likelihood estimate. Biometrika, 70, 343-365.

[3] Berk, R. H. (1972). Consistency and asymptotic normality of MLEs for exponential models. The Annals of Mathematical Statistics, 43, 193-204 .

[4] Doll, R. (1971). The age distribution of cancer: implications for models of carcinogenesis. Journal of the Royal Statistical Society, A, 134, 133-166.

[5] Draper, N. and Smith, H. (1981). Applied regression analysis (2nd ed.). New York: Wiley.

[6] Fahrmeir, L. and Kaufmann, H. (1985). Consistency and asymptotic normality of the maximum likelihood estimators in generalized linear models. The Annals of Statistics, 13, 342-368.

[7] Firth, D. (1991). Generalized linear models. In D. V. Hinkley, N. Reid and E. J. Snell (Eds.), Statistical theory and modelling, 55-86. Chapman and Hall, London.

[8] Freedman, D. A. (2009). Statistical models: Theory and practice. Cambridge University Press. p. 128.

[9] Frome, E. L. (1983). The analysis of rates using Poisson regression model. Biometrics, 39, 665-674. 
[10] Greenlees, W. S., Reece, J. S. and Zieschang, K. D. (1982). Imputation of missing values when the probability of response depends on the variable being imputed. Journal of the American Statistical Association, 77, 251-261.

[11] Huang, L., Chen, M. H. and Ibrahim, J. G. (2005). Bayesian analysis for generalized linear model with nonignorably missing covariates. Biometrics, 61, 767-780.

[12] Haberman, S. J. (1977). Maximum likelihood estimates in exponential response models. Annals of Statistics, 5, 815-841.

[13] Hilbe, J. M. (1994). Generalized linear models. The American Statistician, 48, $255-265$.

[14] Hoffmann, J. P. (2004). Generalized Linear Models; an applied approach, Boston: Allyn and Bacon.

[15] Ibrahim, J. G. (1990). Incomplete data in generalized linear models. Journal of the American Statistical Association, 85, 765-769.

[16] Ibrahim, J. G., Chen, M. H., Lipsitz, S. R. and Hemng, A. H. (2005). Missingdata methods for generalized linear models: A comparative review. Journal of the American Statistical Association, 100, 332-346.

[17] Ibrahim, J. G. and Lipsitz, S. R. (1999). Missing covariates in generalized linear models when the missing data mechanism is non-ignorable. Journal of the Royal Statistical Society, B, 61:173-190.

[18] Lane, P. W. (2002). Generalized linear models in soil science. European Journal of Soil Science, 53, 241-251. 
[19] Little, R. J. A. and Rubin, D. B. (2002). Statistical Analysis With Missing Data, Second Edition. Wiley, New York.

[20] Little, R. J. A. (1992) Regression with missing X's: a review. Journal of the American Statistical Association, 87:1227-1237.

[21] McCullagh, P. and Nelder, J. A. (1989). Generalized Linear Models, Second Edition. Chapman and Hall, London.

[22] Nelder, J. A. and Wedderburn, R. W. M. (1972). Generalized linear models. Journal of the Royal Statistical Society, A, 135, 370-384.

[23] Janke, S. J. and Tinsley, F. C. ( 2005). Book Review: Introduction to Linear Models and Statistical Inference. NJ : Wiley.

[24] Sinha, S. K.(2008). Robust methods for generalized linear models with nonignorable missing covariates. The Canadian Journal of Statistics, 36, 277-299.

[25] Shi, X., Zhu, H. and Ibrahim, J. G. (2009). Local Influence for generalized linear models with Missing Covariates. Biometrics, 65, 1164-1174.

[26] Tang, G., Little, R. J. A., and Raghunathan, T. E. (2003). Analysis of multivariate missing data With nonignorable nonresponse. Biometrika, 90, 747-764.

[27] Wu, H. and Wu, L. (2001). A multiple imputation method for missing covariates in non-linear mixed-effects models with application to HIV dynamics. Statistics in medicine, 20: 1755-1769 .

[28] Wei, G. C. G. and Tanner, M. A. (1990): A Monte Carlo Implementation of the EM algorithm and the poor man's data augmentation algorithms. Journal of the 
American Statistical Association, 85, 699-704.

[29] WU, Z. (2005). Generalized linear models in family studies. Journal of Marriage and Family 67, 1029-1047.

[30] Yang, X., Belin, T. R. and Boscardin, W. J. (2005). Imputation and variable selection in linear regression models with missing covariates. Biometrics, 61, 498506.

[31] Zhao, J. and Shao, J. (2015). Semiparametric pseudo-likelihoods in generalized linear models with nonignorable missing Data. Journal of the American Statistical Association, 110, 1577-1590. 


\section{Appendix A}

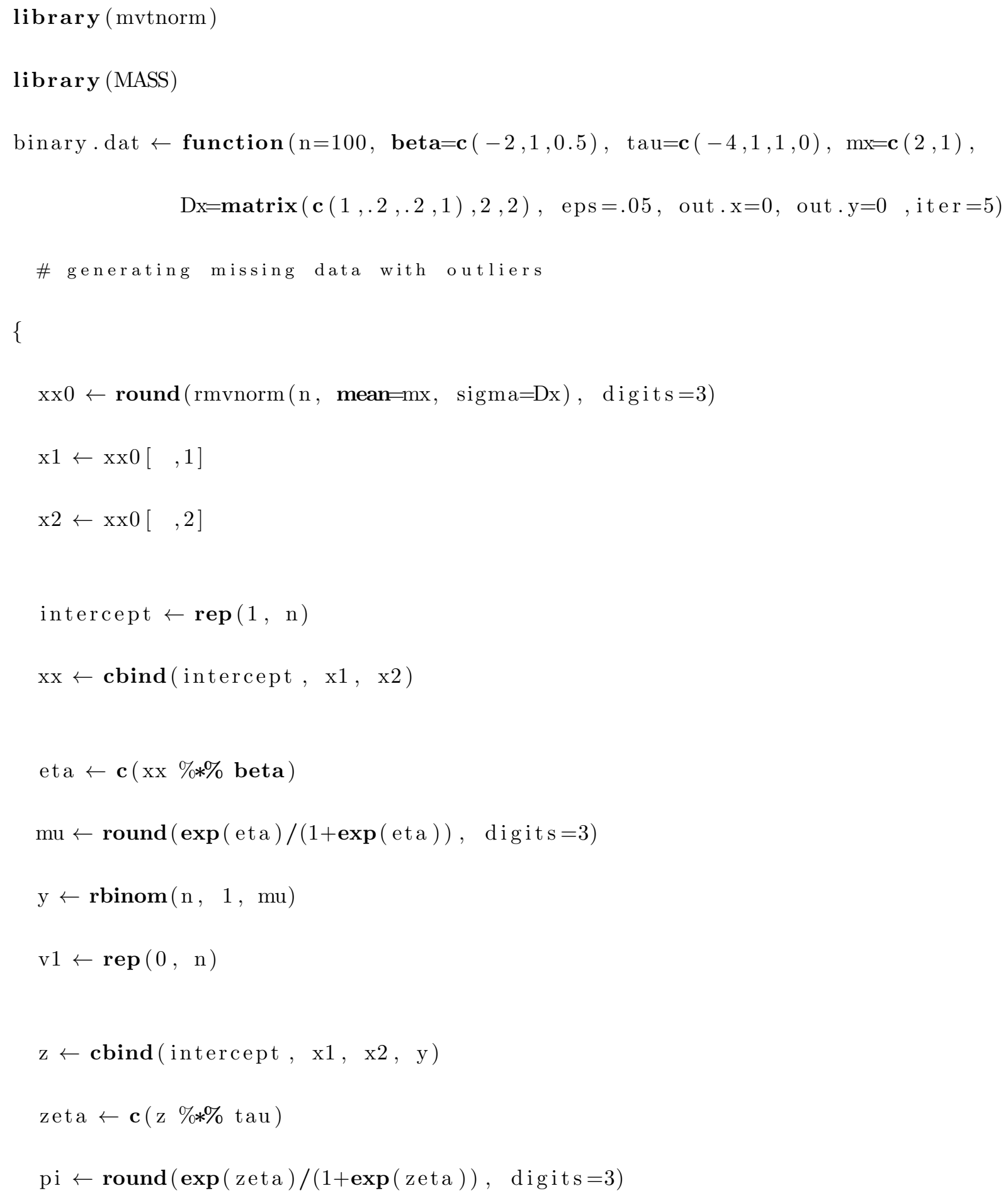




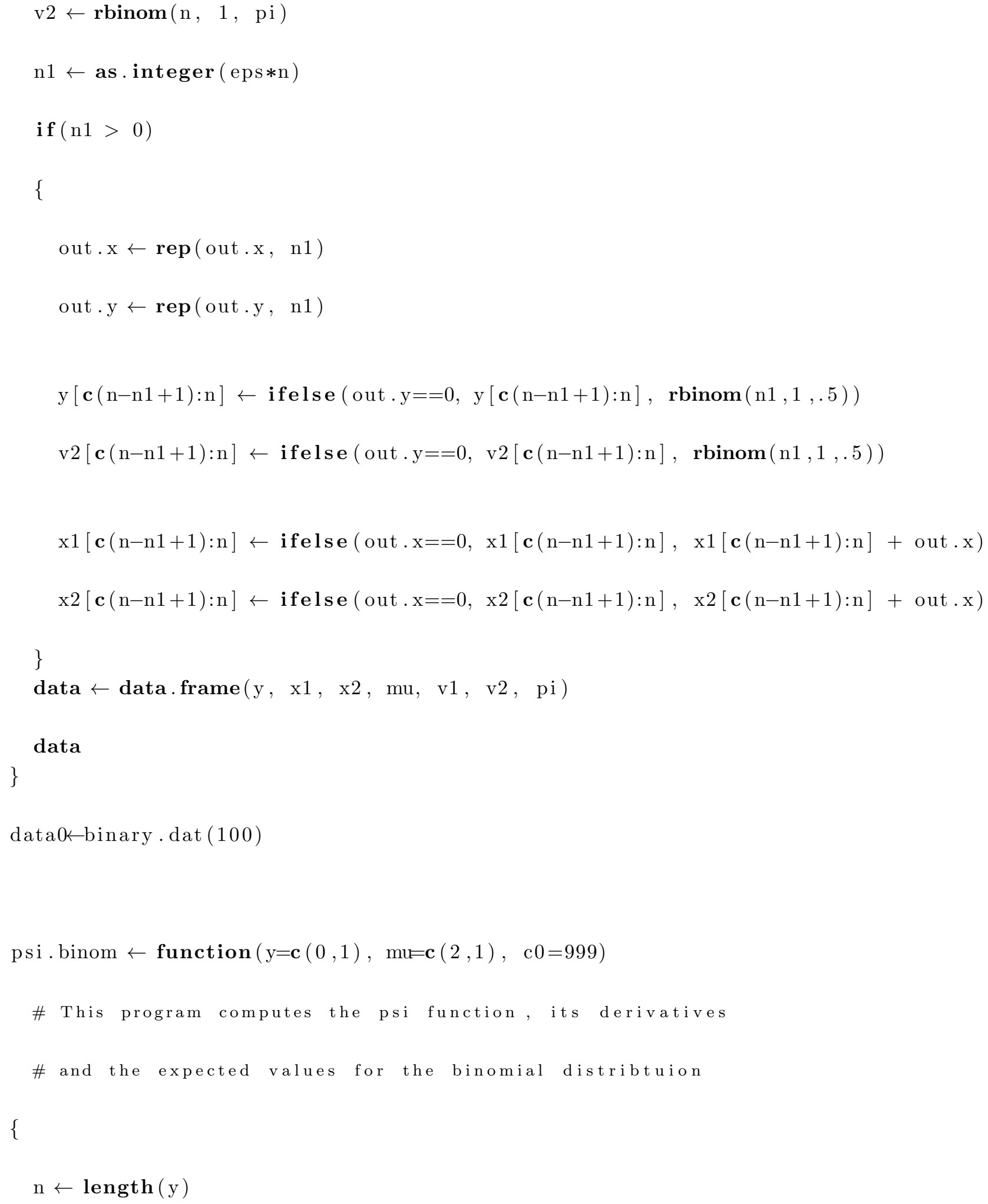




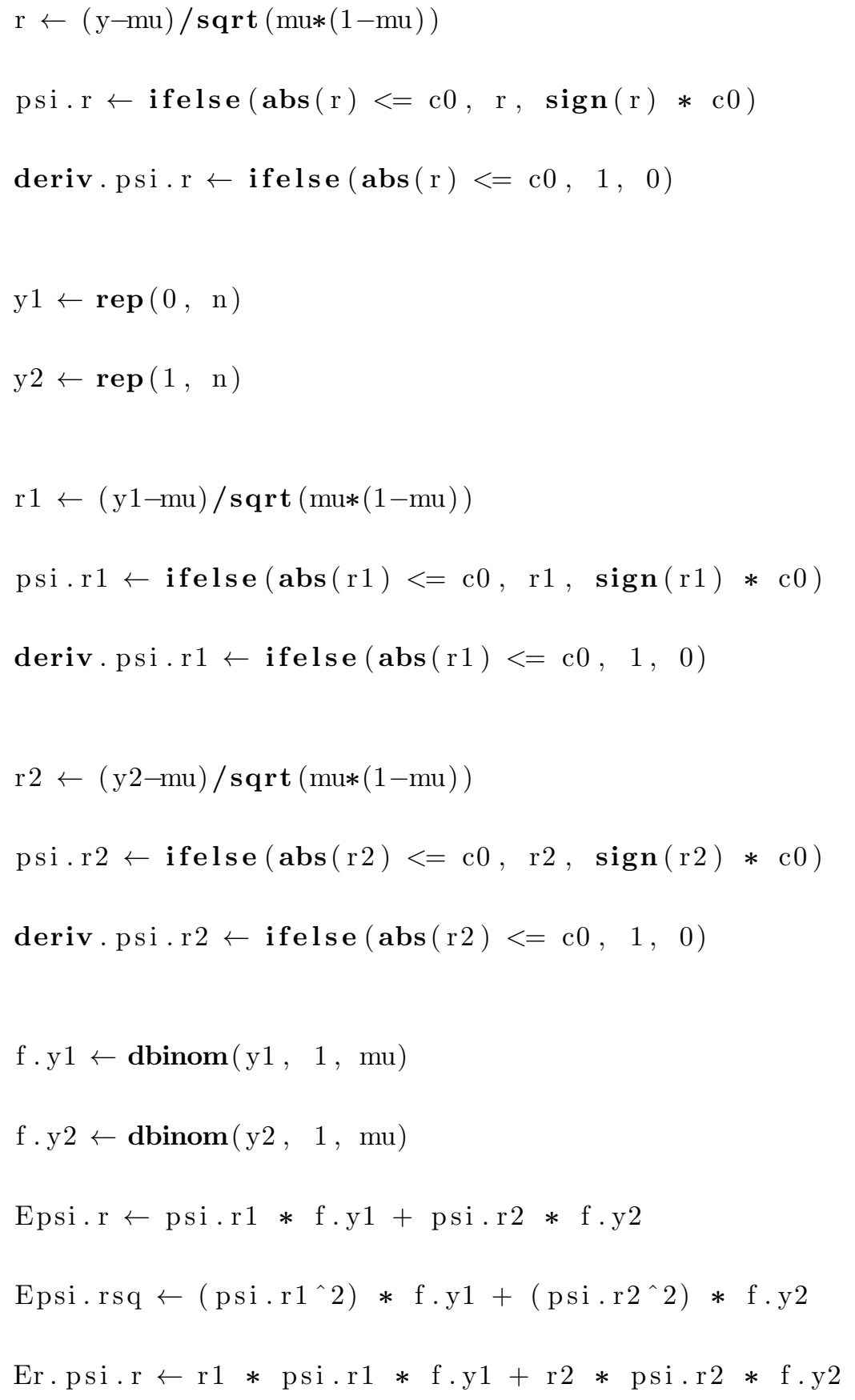




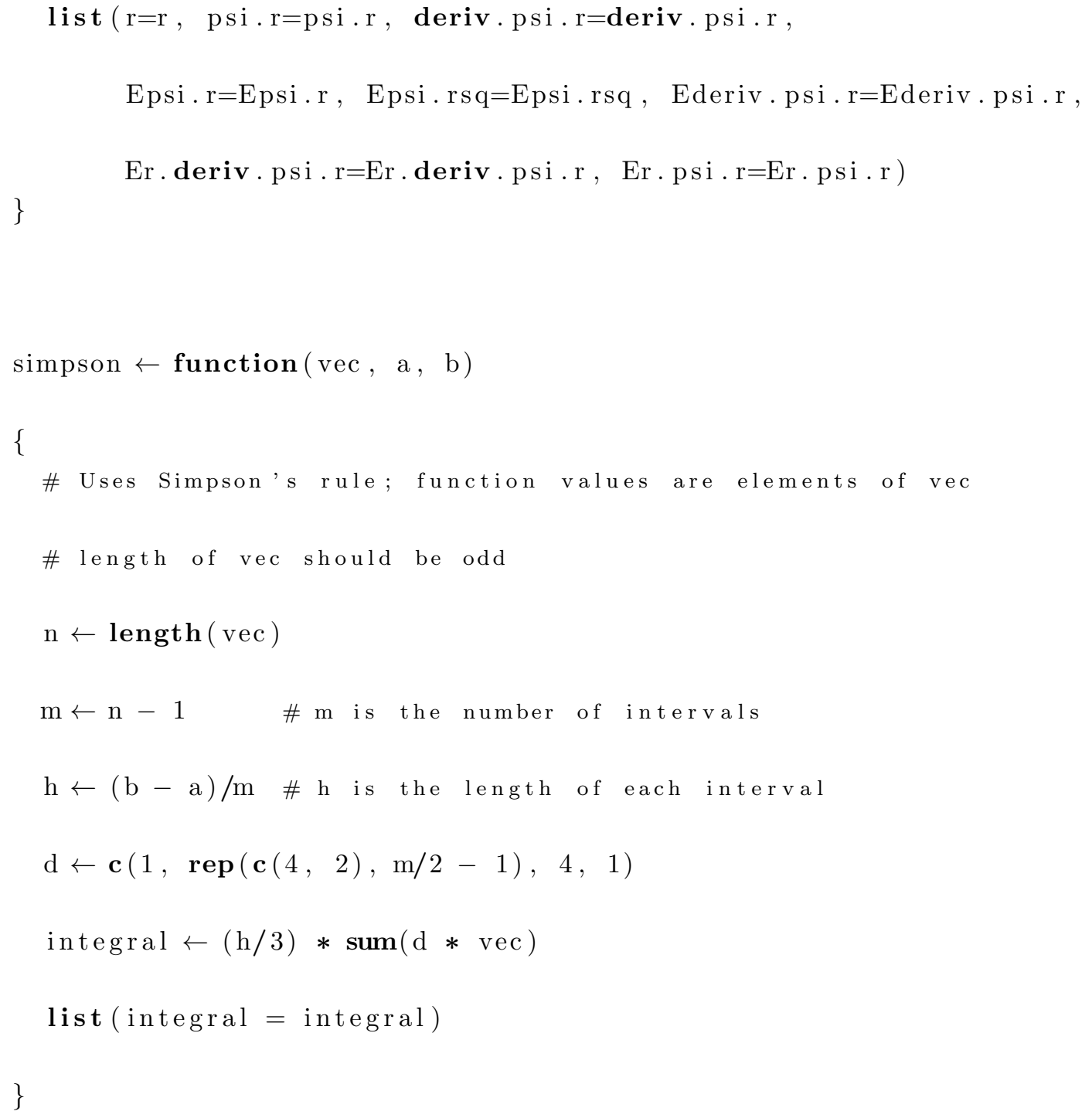




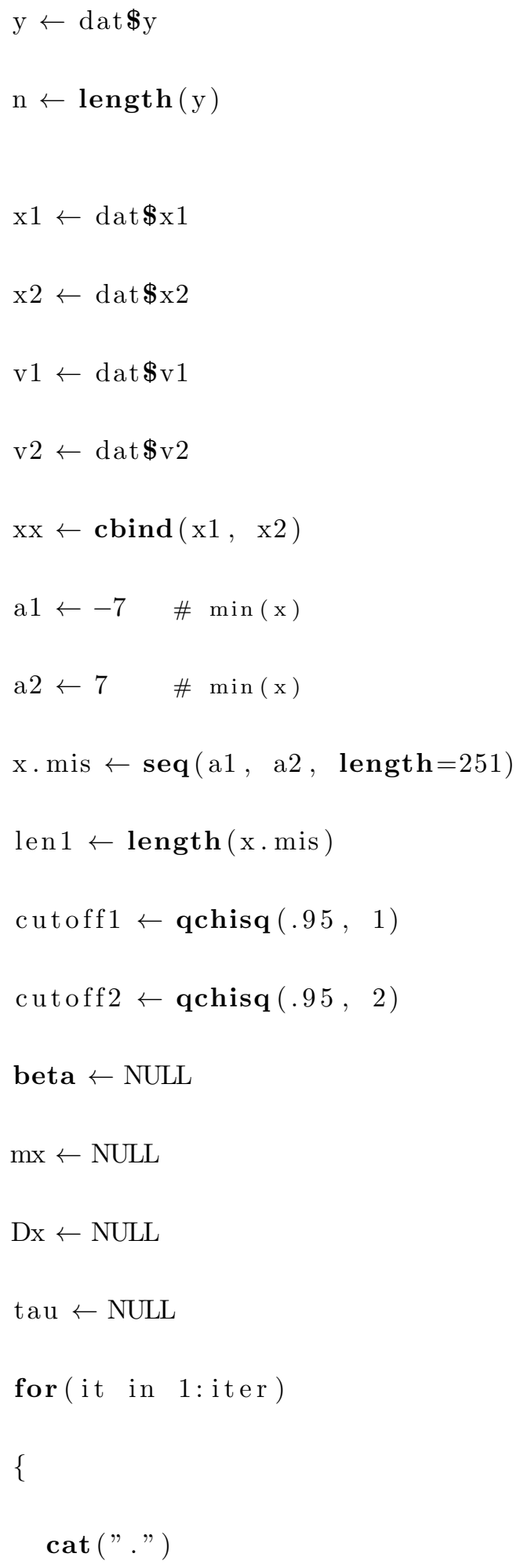




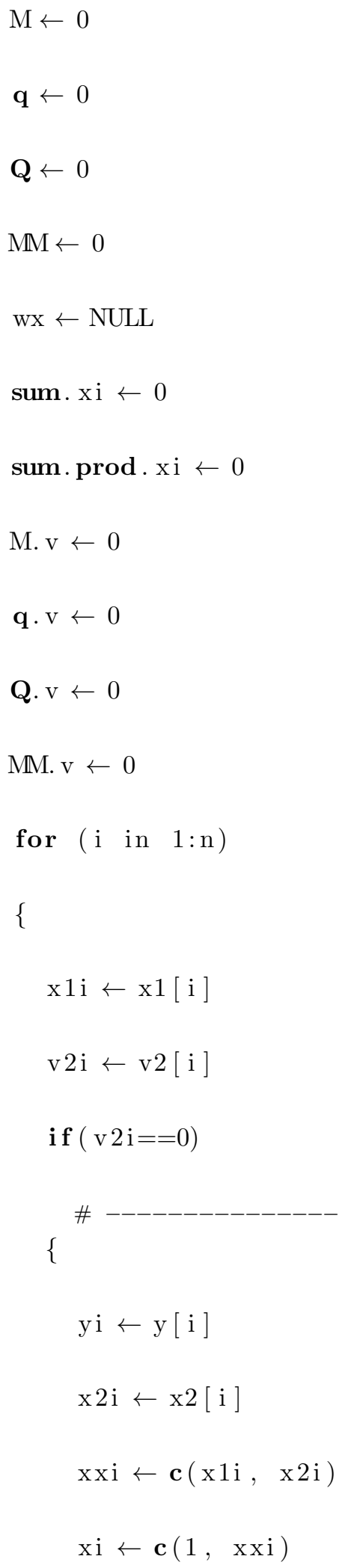




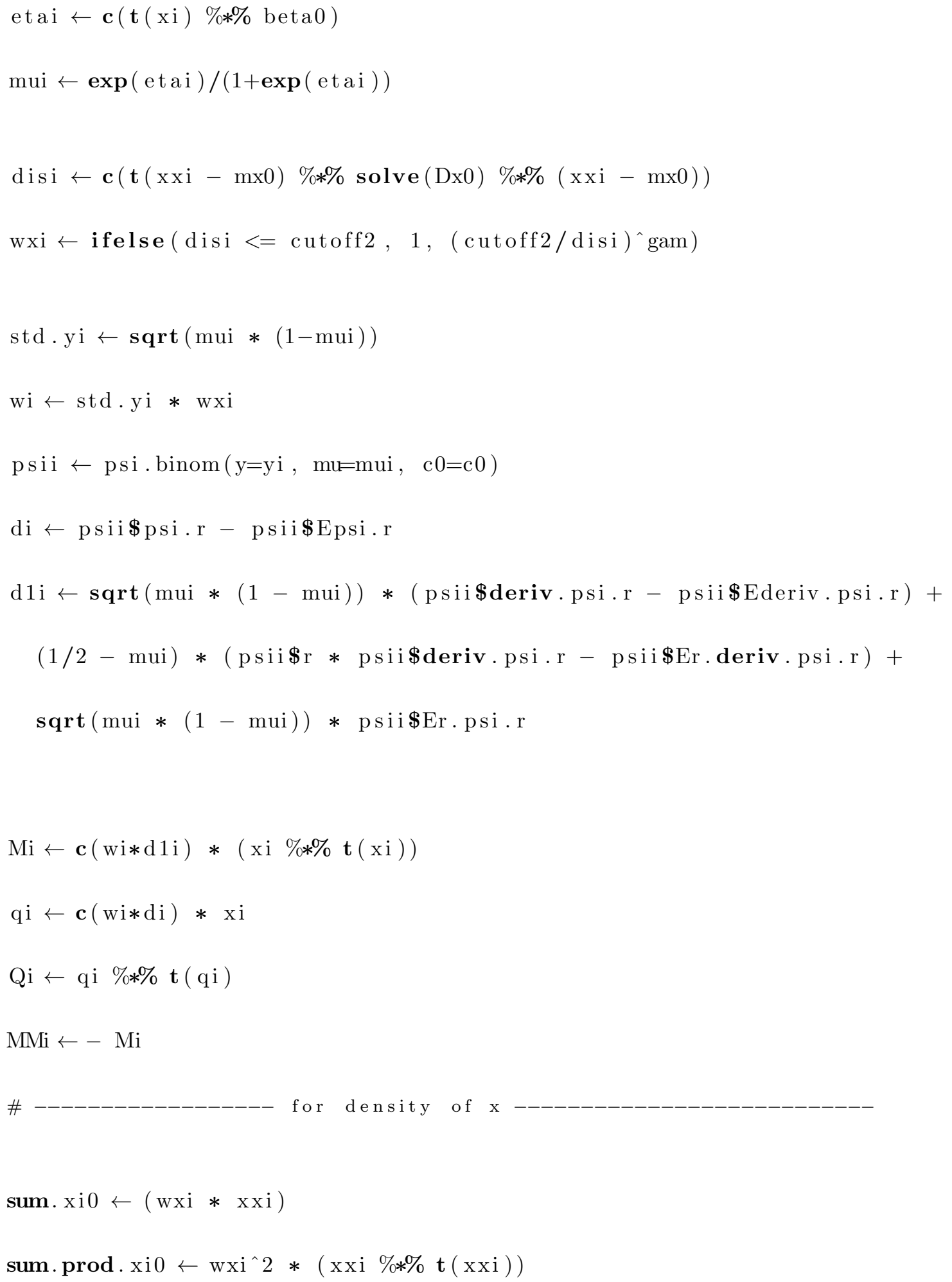




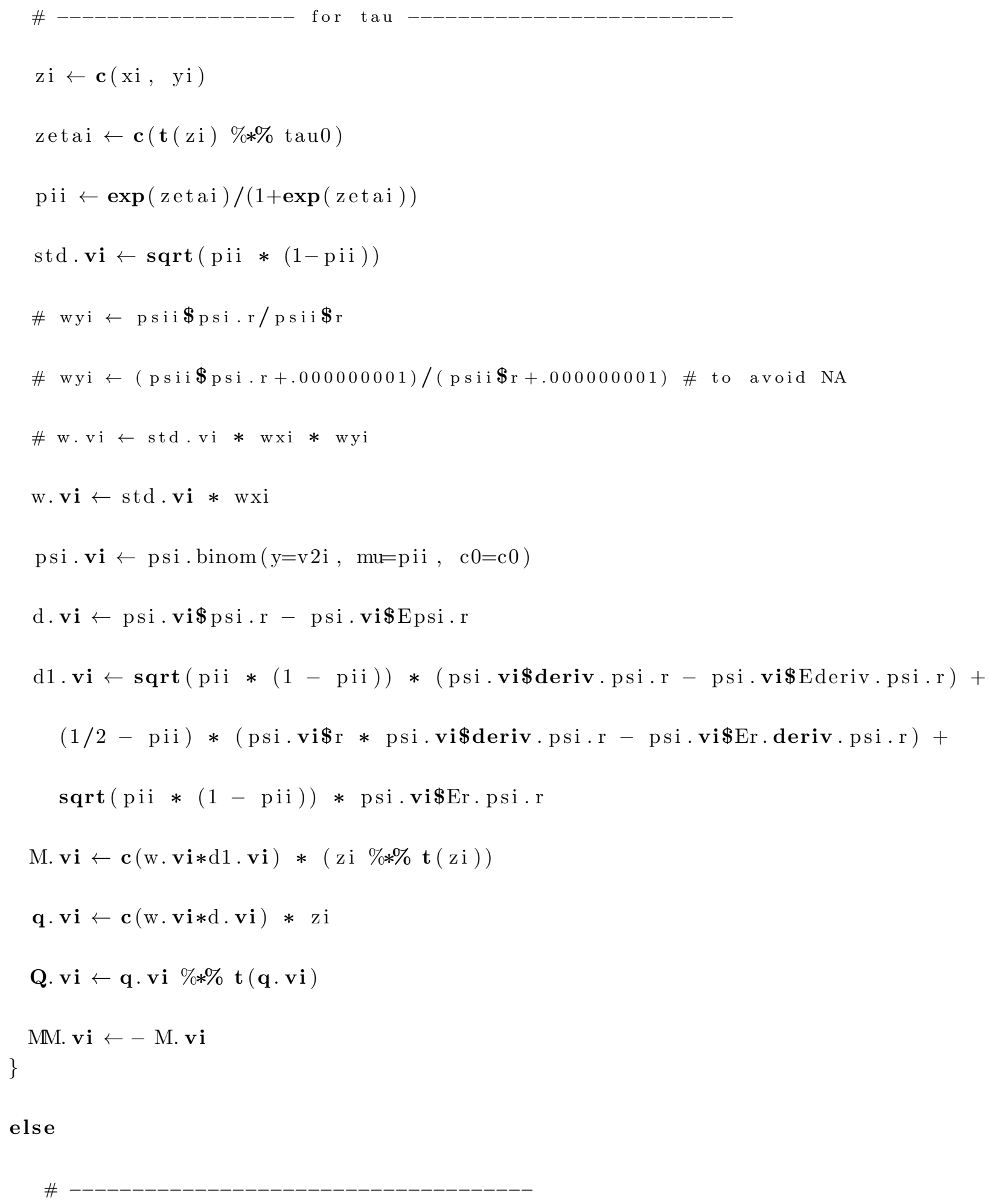




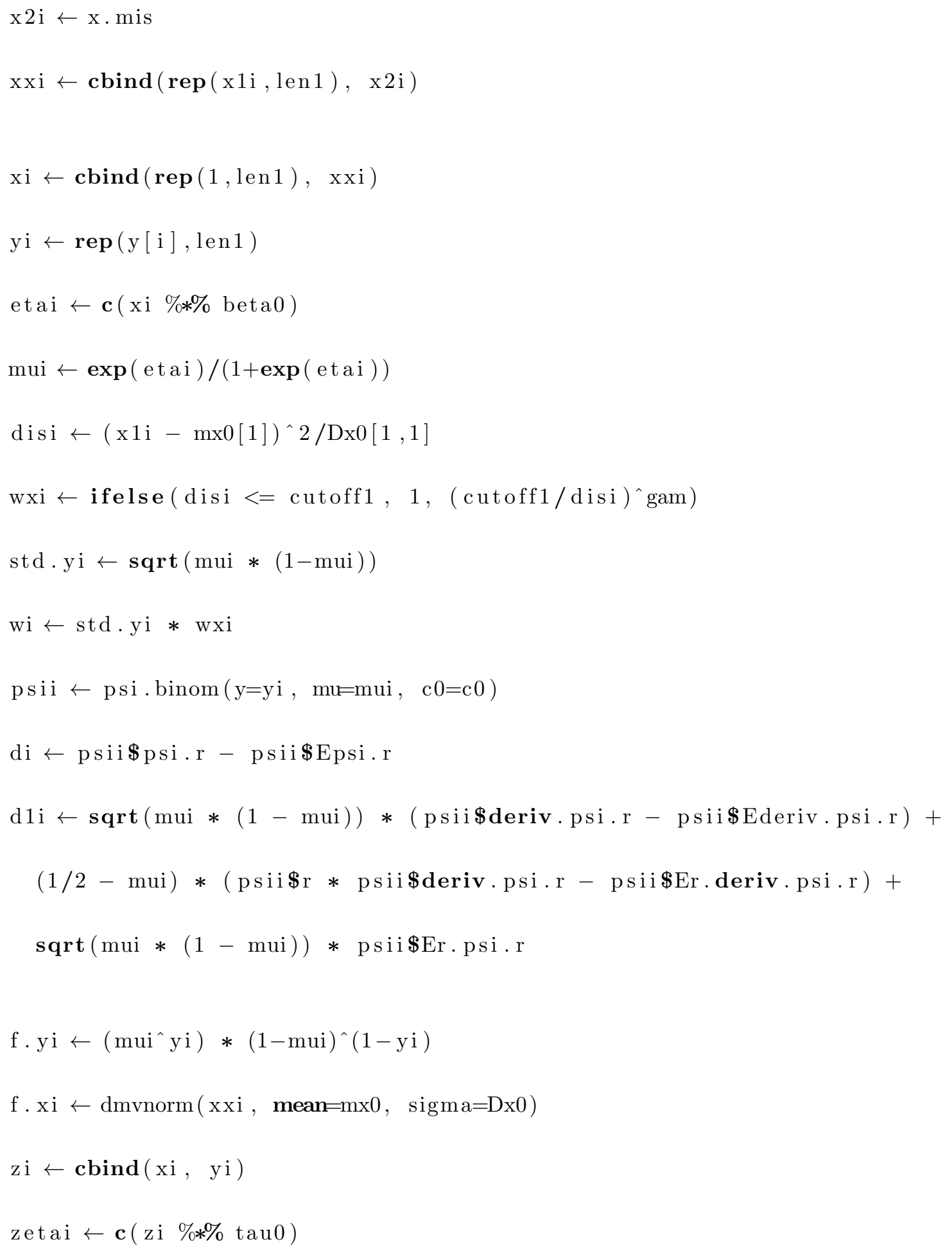




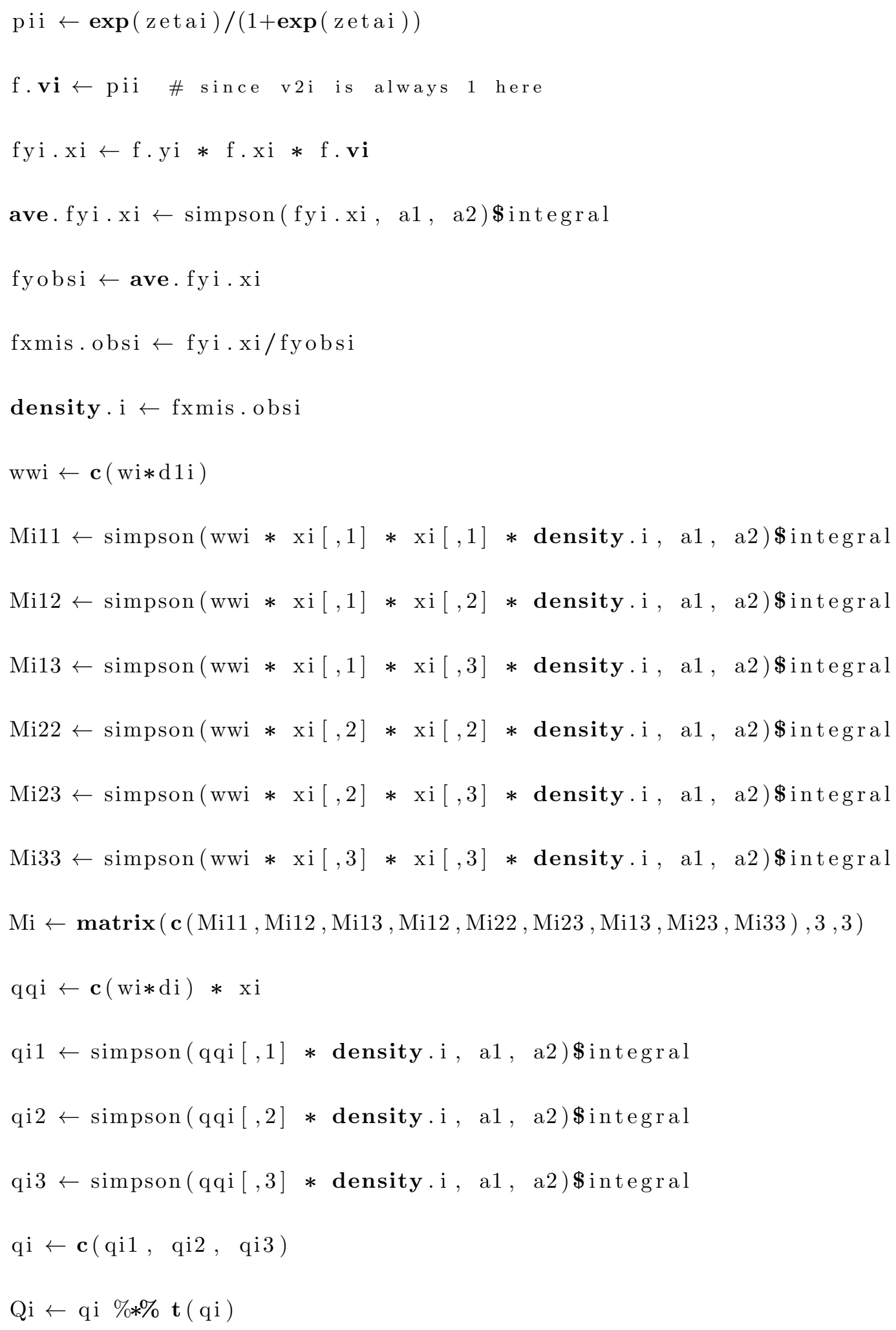




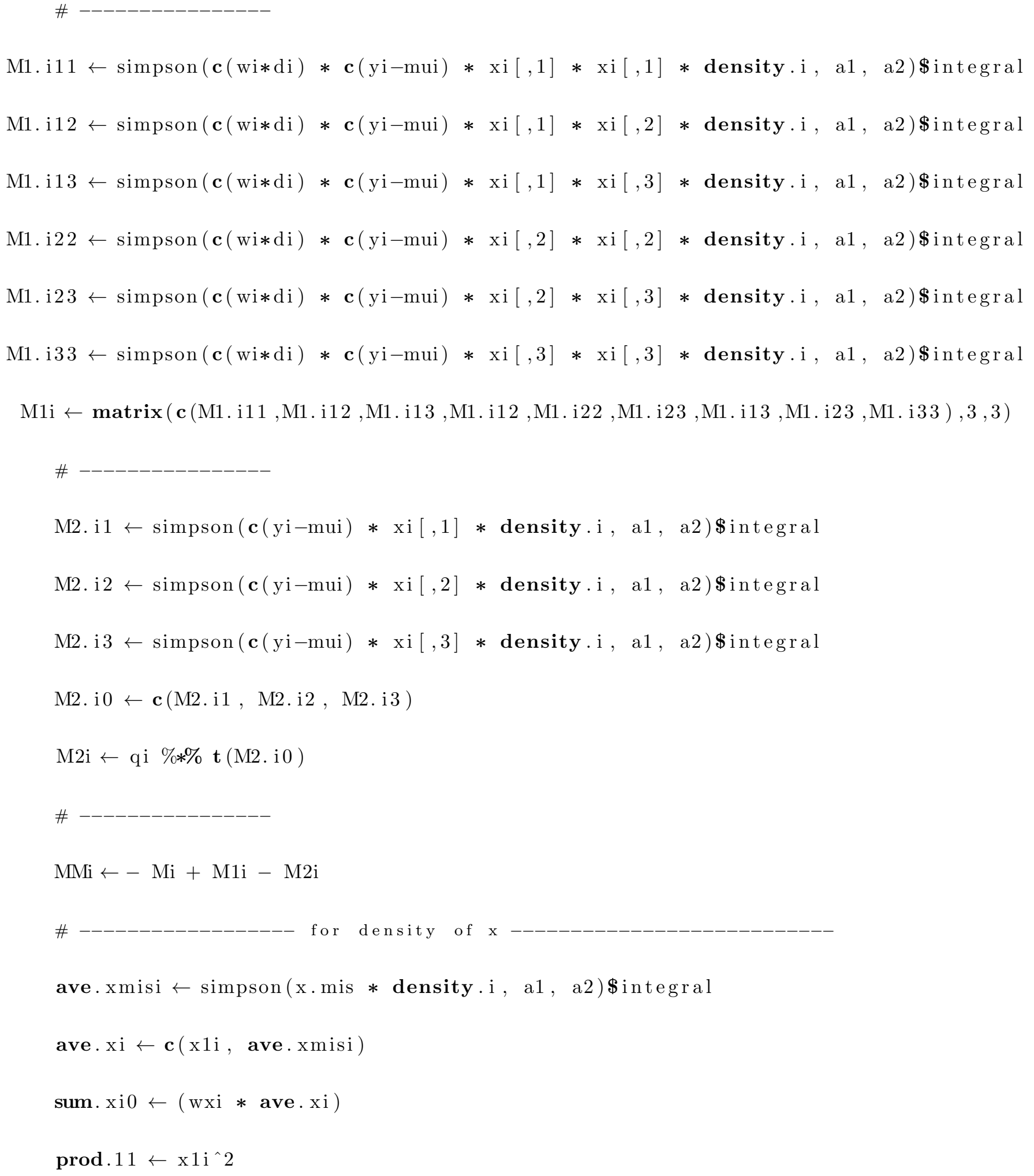$$
\text { M2. } 11 \leftarrow \operatorname{simpson}(\mathbf{c}(\mathrm{yi}-\mathrm{mui}) * \mathrm{xi}[, 1] * \operatorname{density.i}, \text { a1, a2)\$integral }
$$$$
\text { M2. i } 2 \leftarrow \operatorname{simpson}(\mathbf{c}(\mathrm{yi}-\mathrm{mui}) * \mathrm{xi}[, 2] * \text { density.i, a1, a2)\$integral }
$$$$
\text { M2. i } 3 \leftarrow \operatorname{simpson}(\mathbf{c}(\mathrm{yi}-\mathrm{mui}) * \mathrm{xi}[, 3] * \text { density.i, a1, a2)\$integral }
$$$$
\mathrm{M} 2 . \mathrm{i} 0 \leftarrow \mathbf{c}(\mathrm{M} 2 . \mathrm{i} 1, \mathrm{M} 2 . \mathrm{i} 2, \mathrm{M} 2 . \mathrm{i} 3)
$$$$
\mathrm{M} 2 \mathrm{i} \leftarrow \text { qi } \% * \% \mathrm{t}(\mathrm{M} 2 . \mathrm{i} 0)
$$$$
\text { \# }
$$$$
\mathrm{MMi} \leftarrow-\mathrm{Mi}+\mathrm{M} 1 \mathrm{i}-\mathrm{M} 2 \mathrm{i}
$$$$
\text { \# }
$$$$
\text { ave.xmisi } \leftarrow \operatorname{simpson}(x \cdot \text { mis } * \text { density.i, a1, a2)\$integral }
$$$$
\text { ave. } x i \leftarrow \mathbf{c}(x 1 i, \text { ave. } x \text { misi })
$$$$
\text { sum. xi } 0 \leftarrow(\text { wxi } * \text { ave. xi })
$$$$
\operatorname{prod} .11 \leftarrow \mathrm{x} 1 \mathrm{i}^{\wedge} 2
$$ 


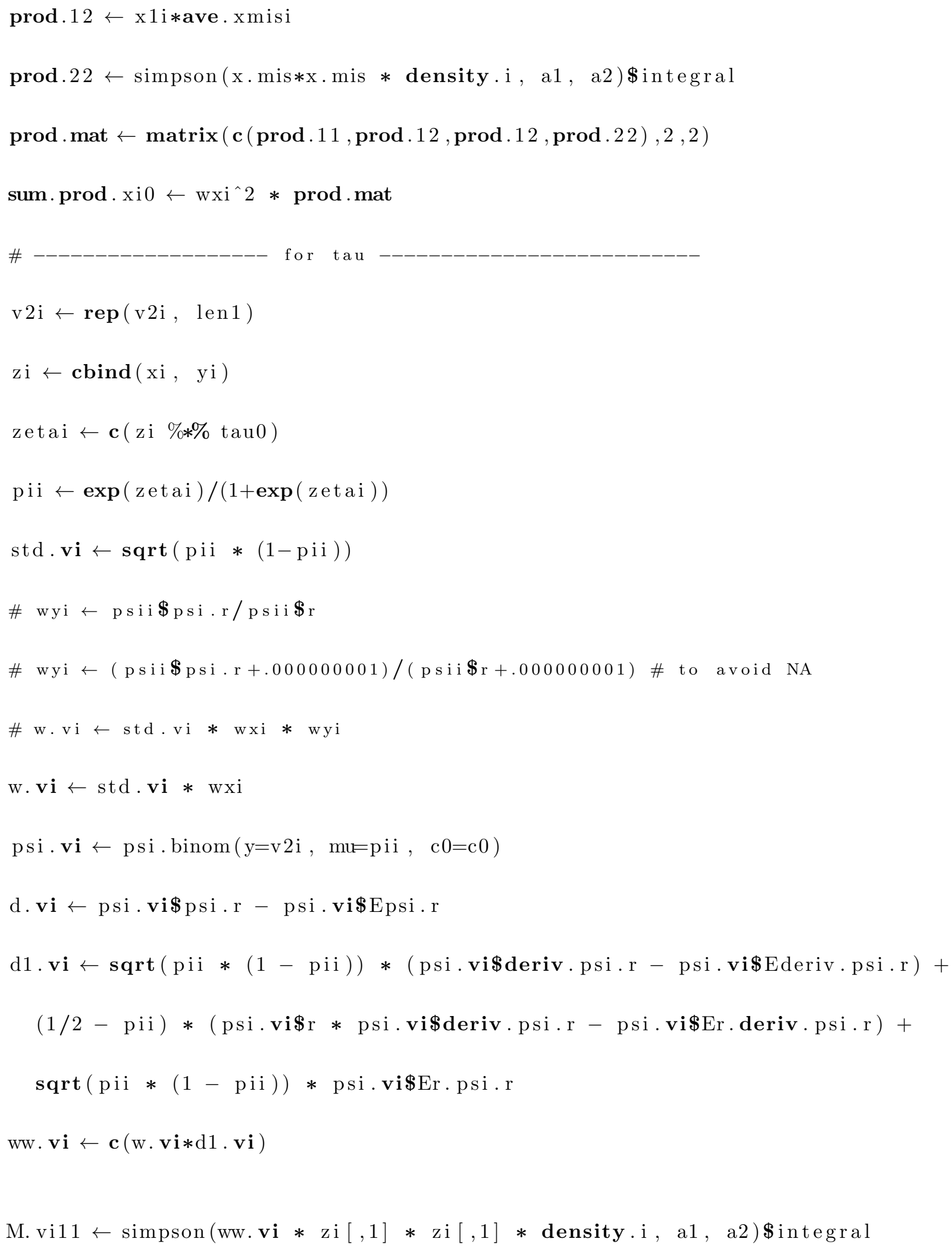




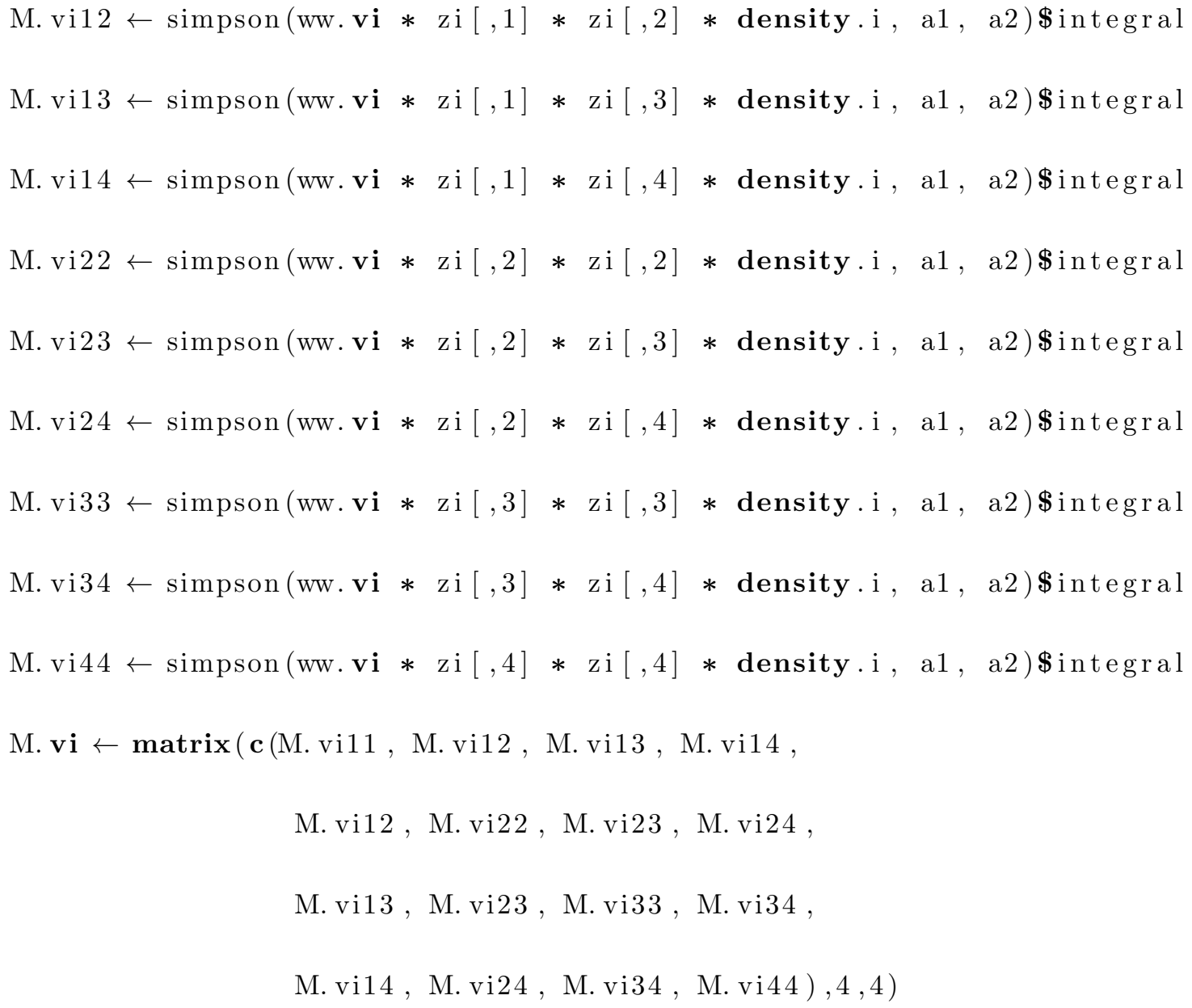




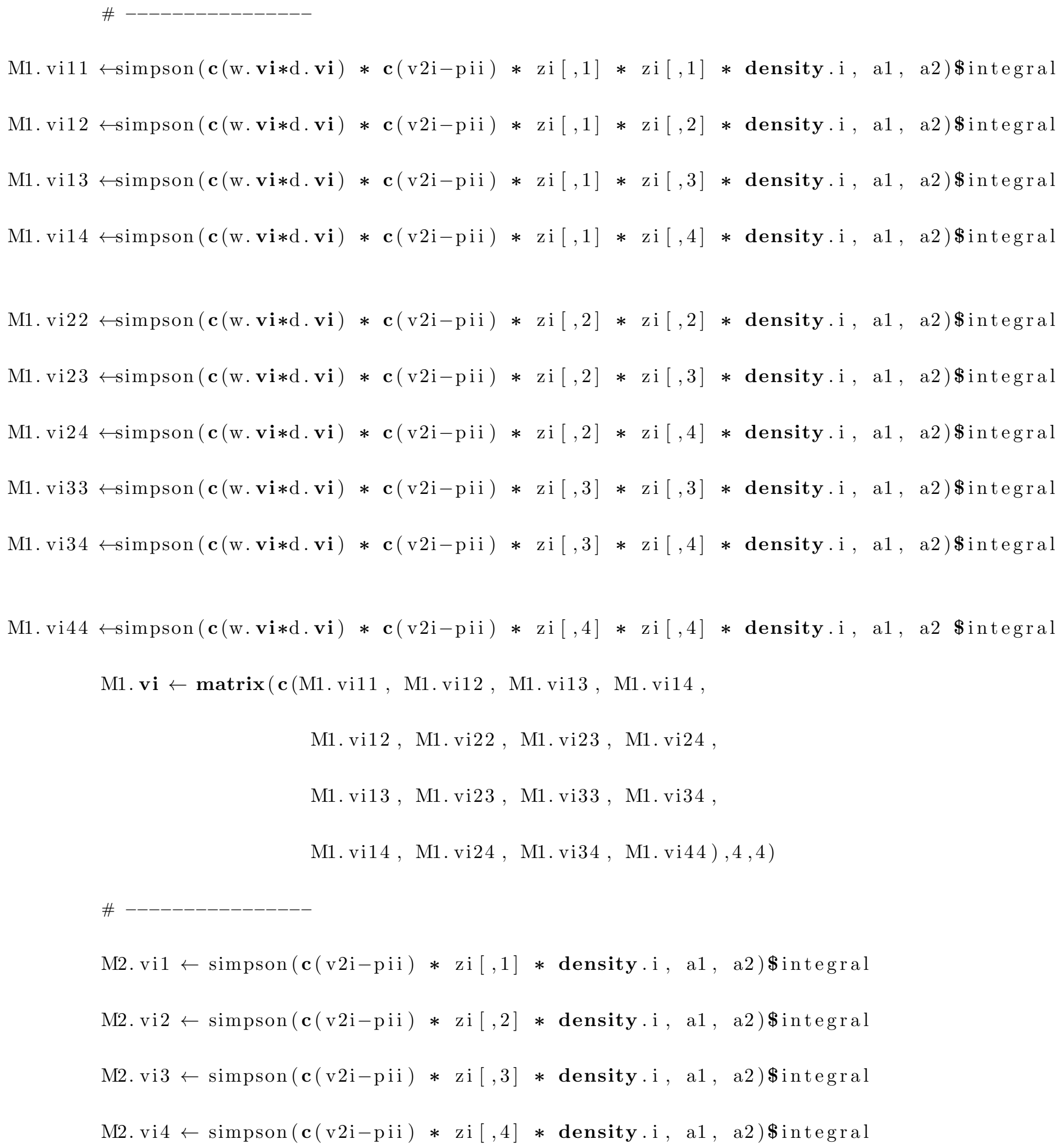




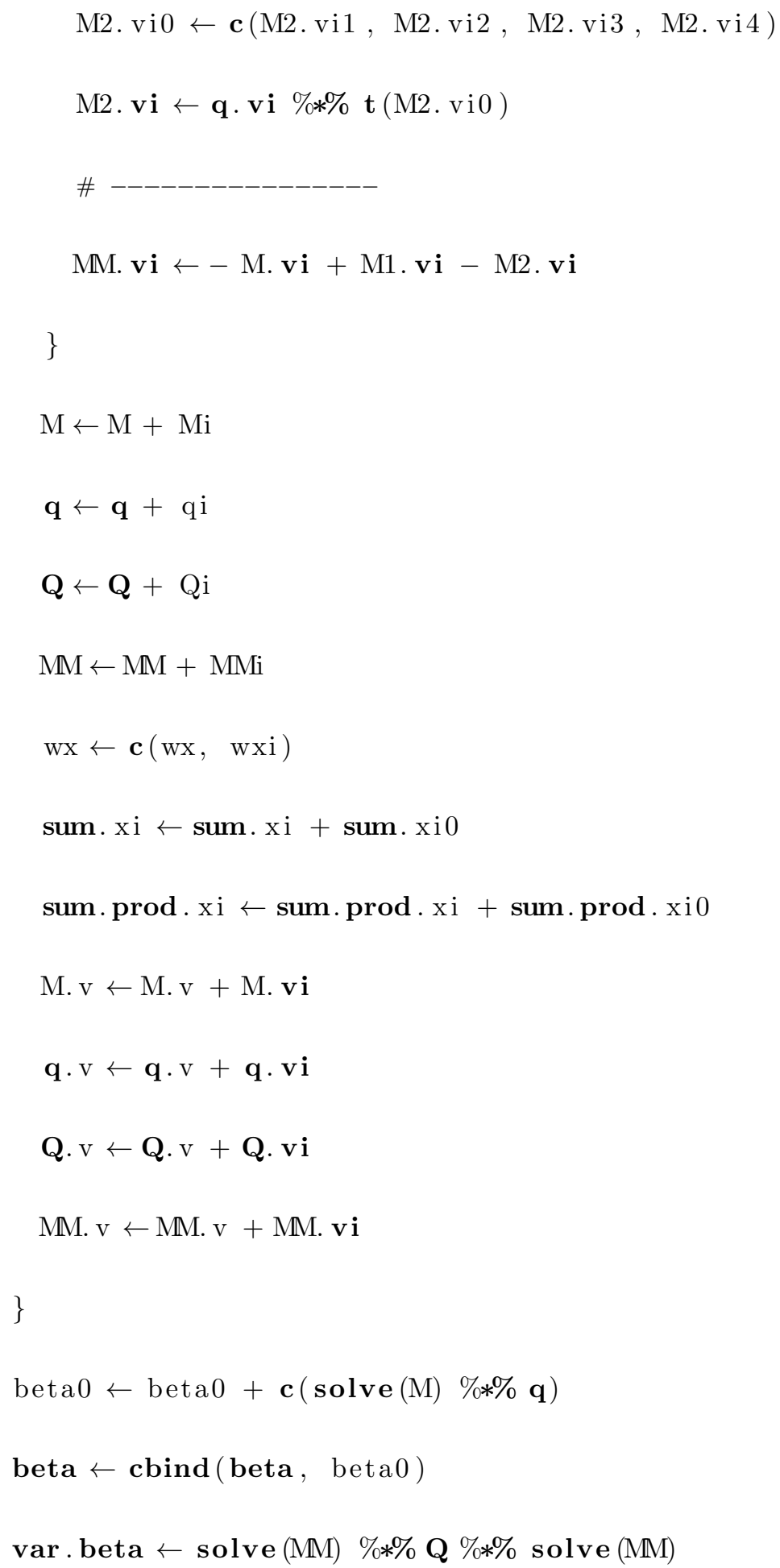




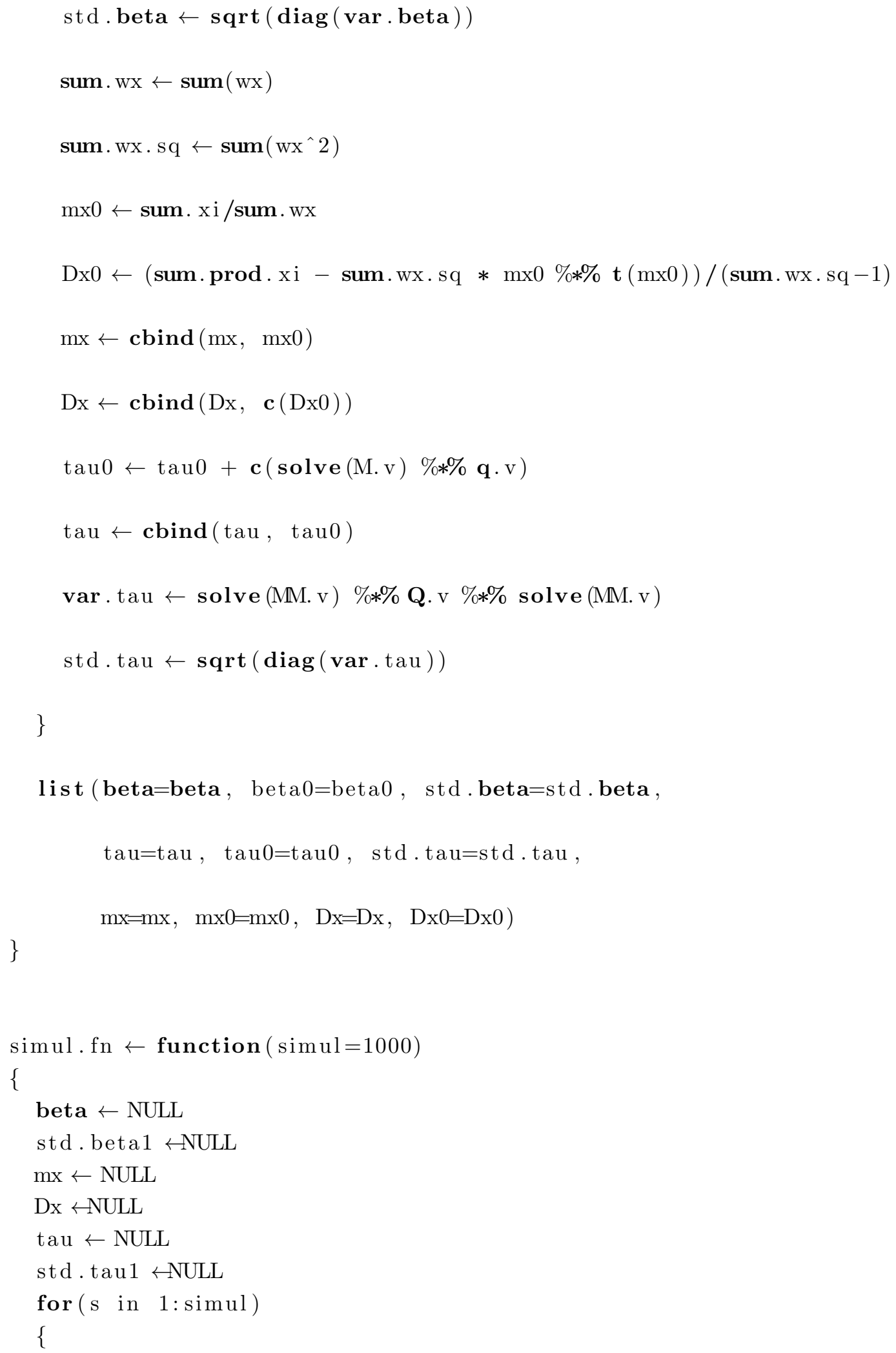




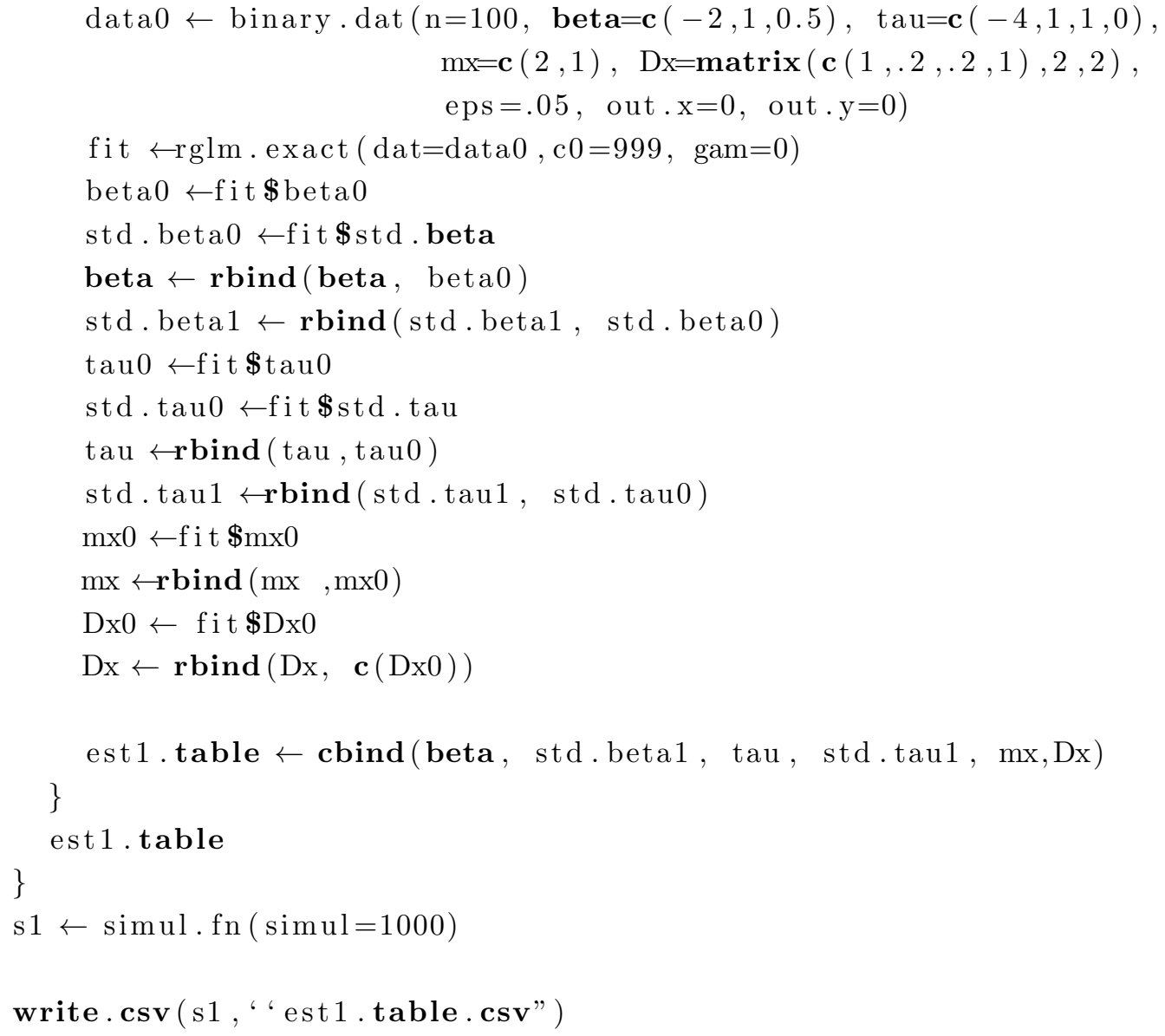




\section{Appendix B}

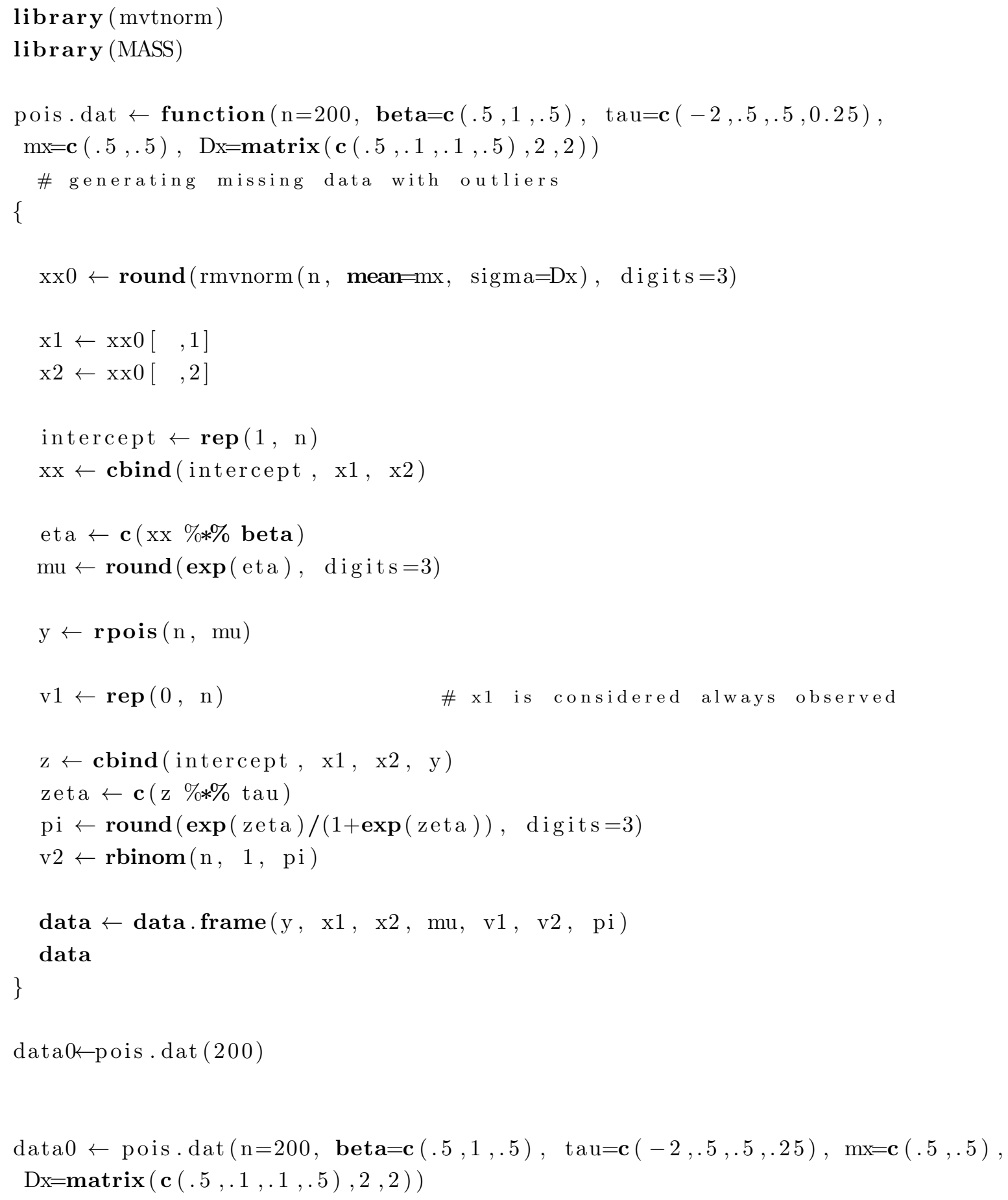




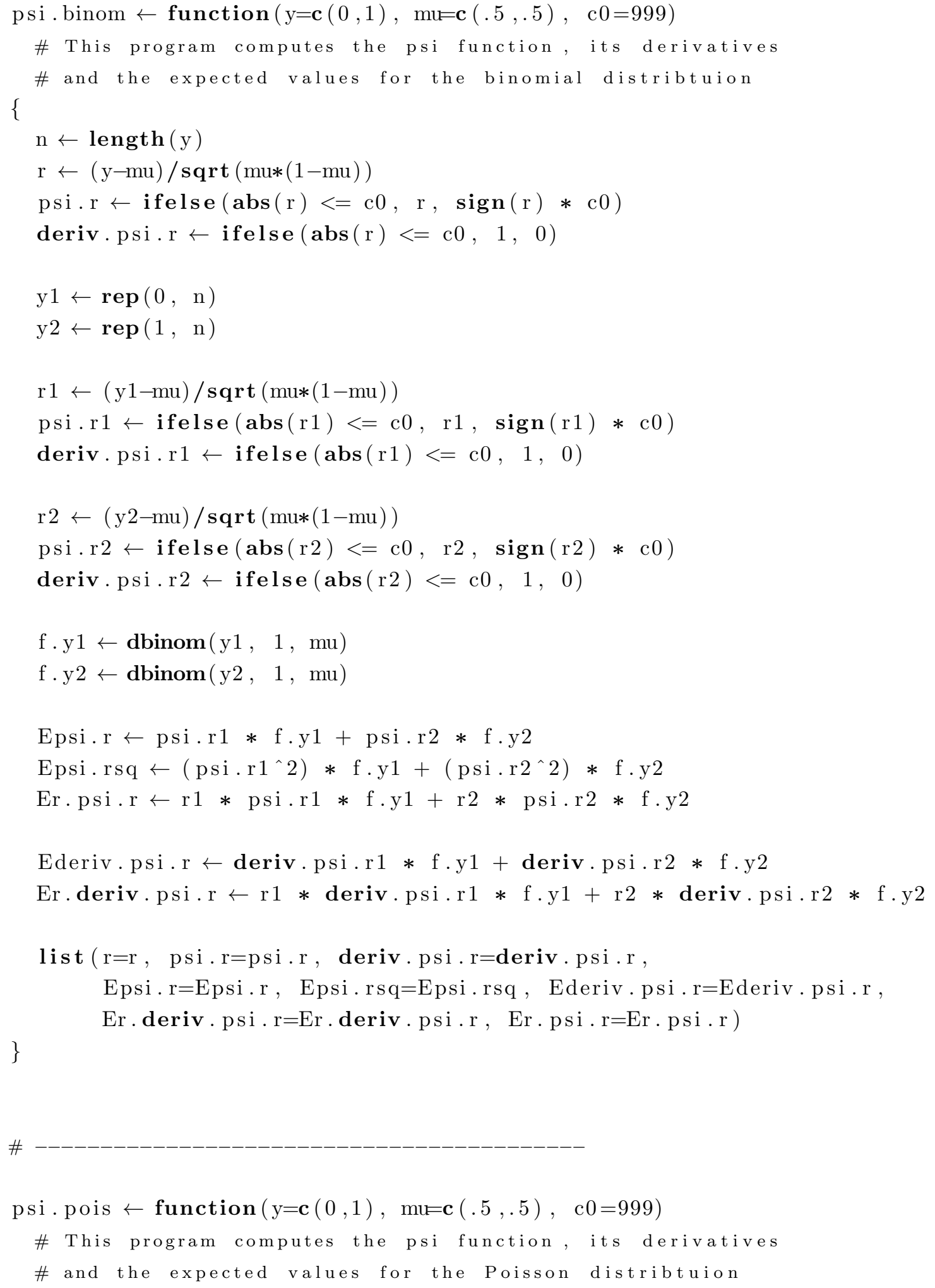




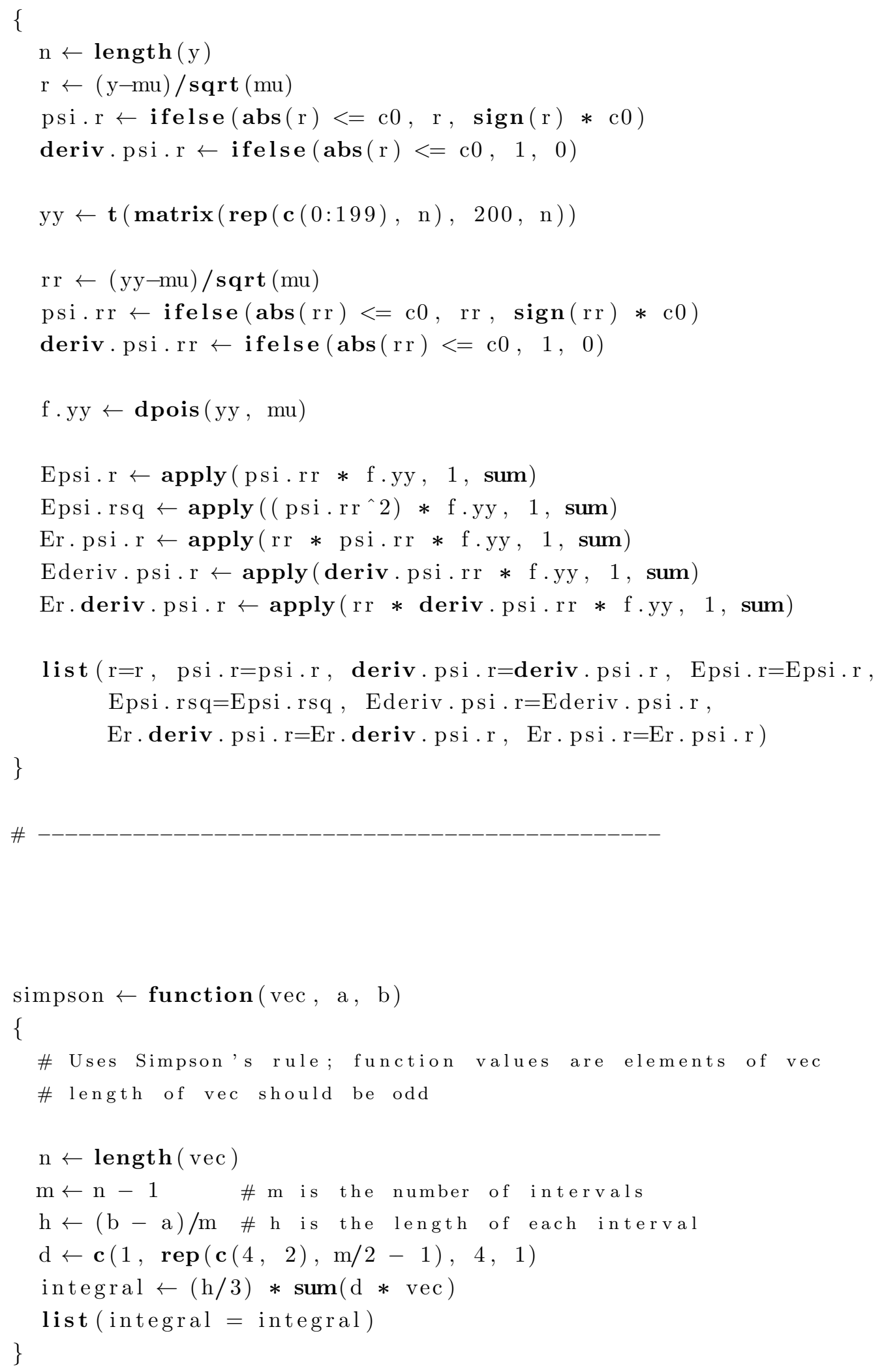




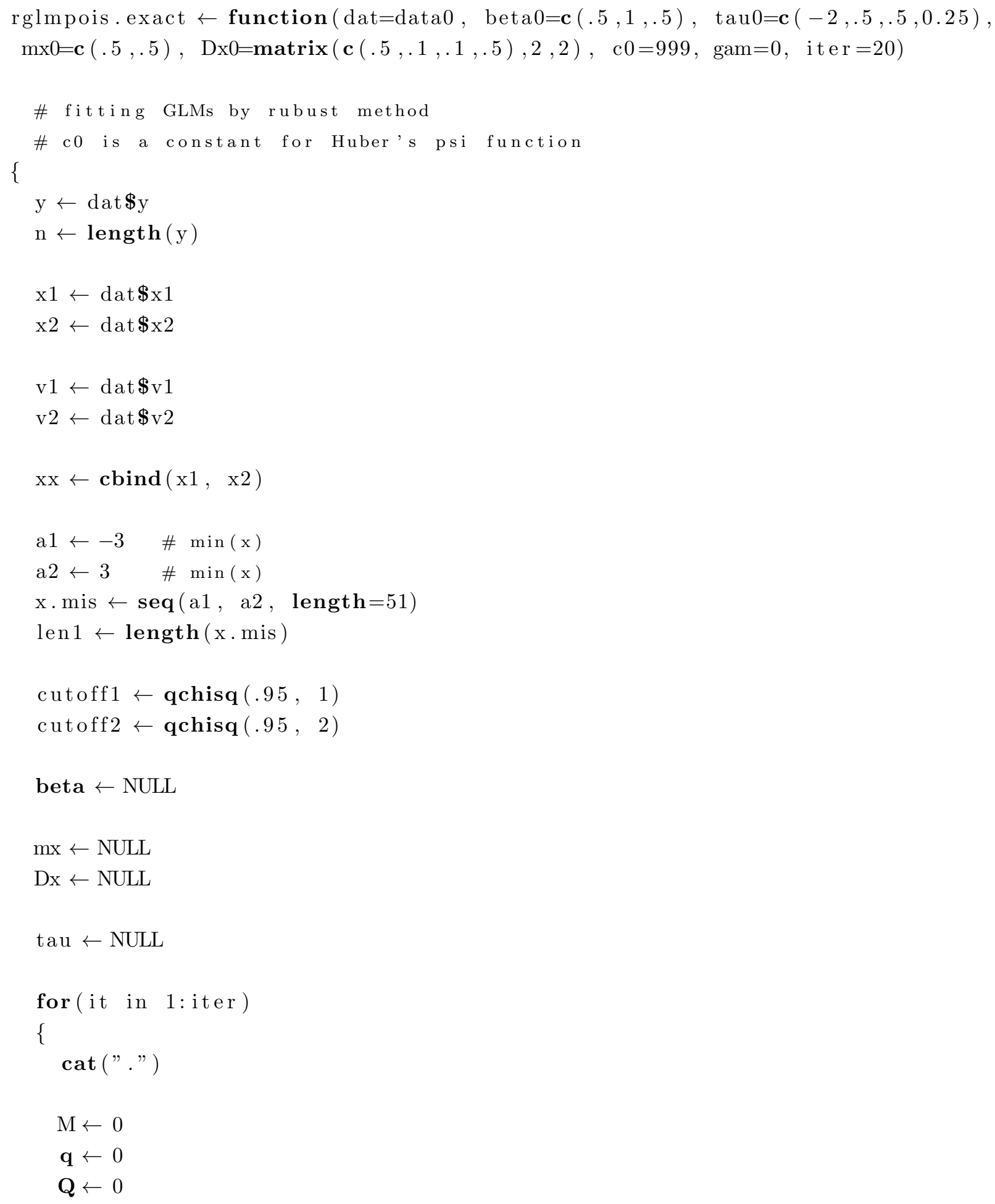




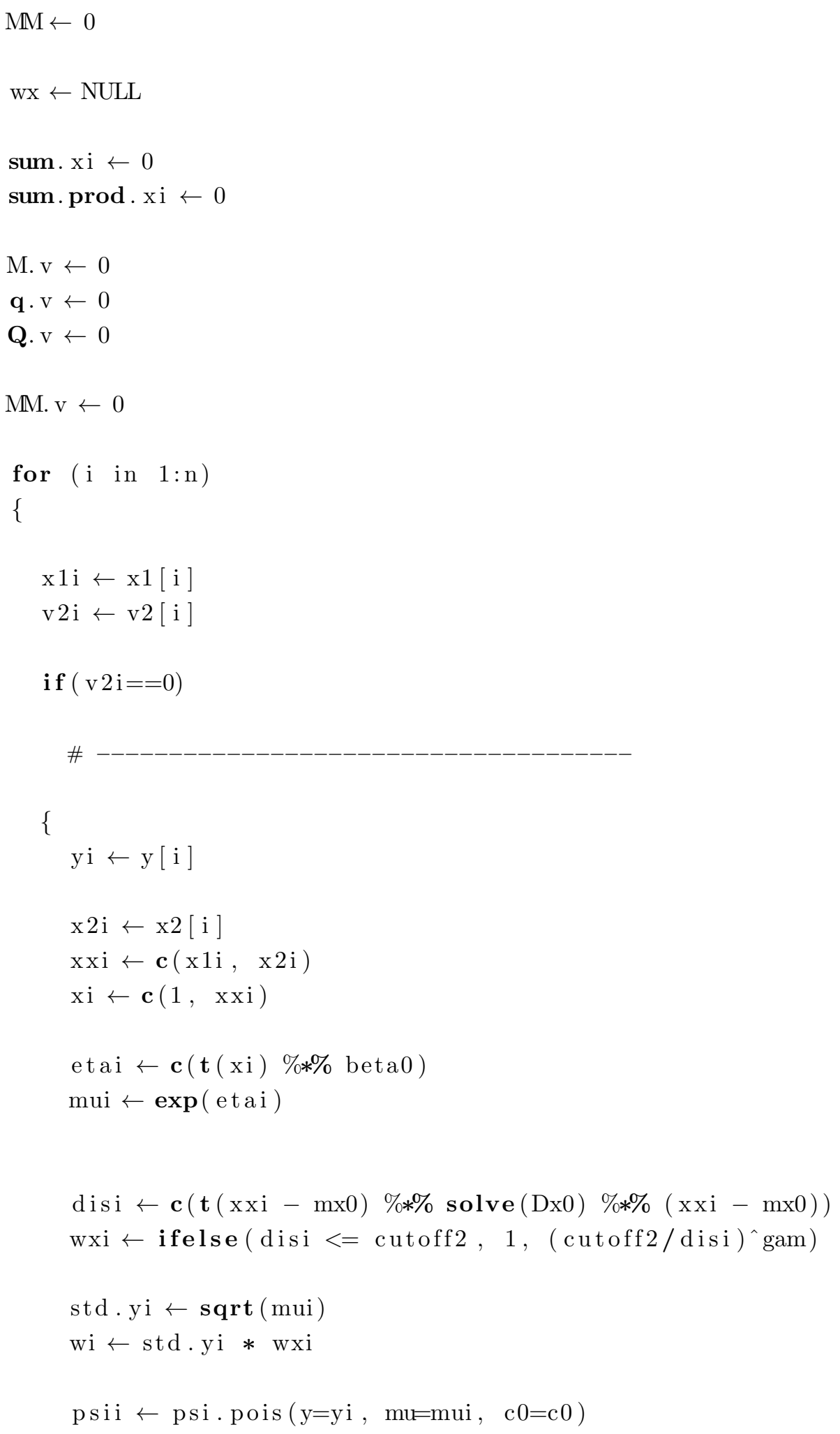




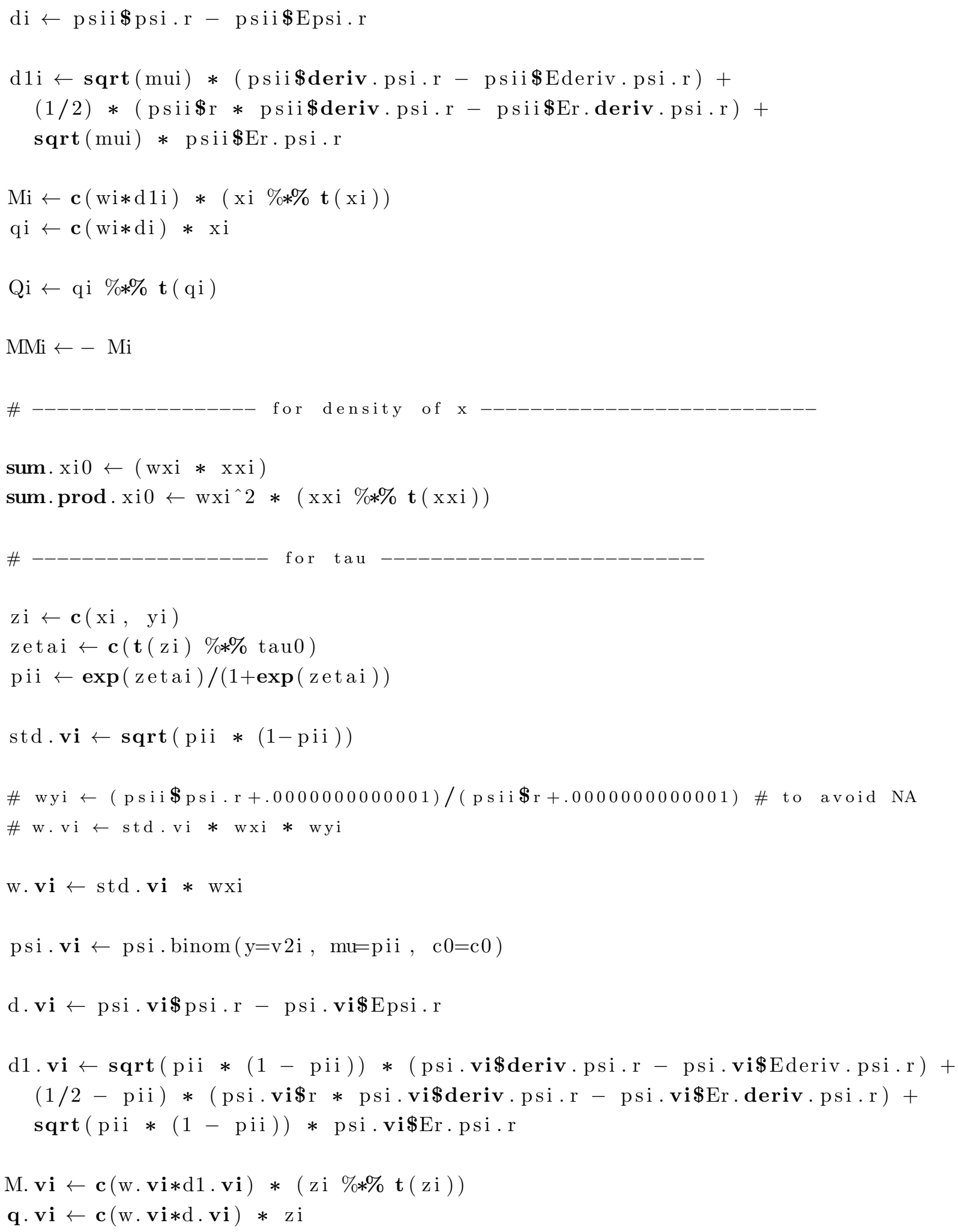




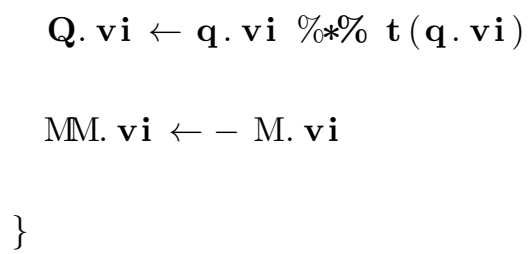




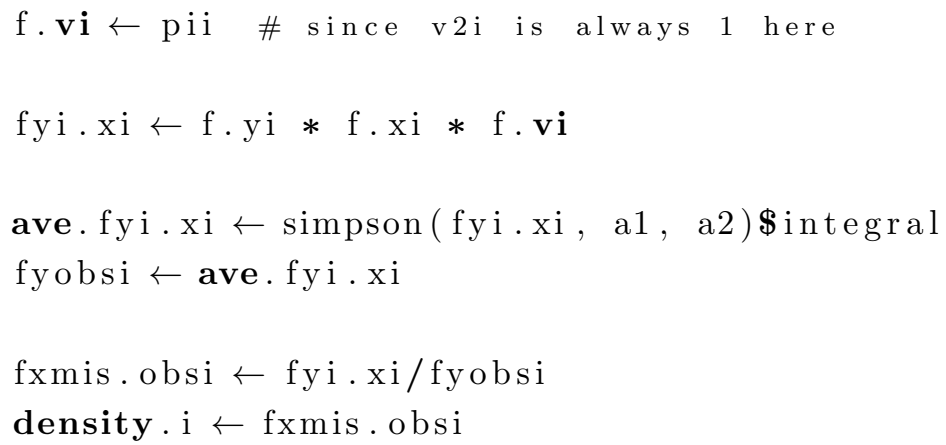




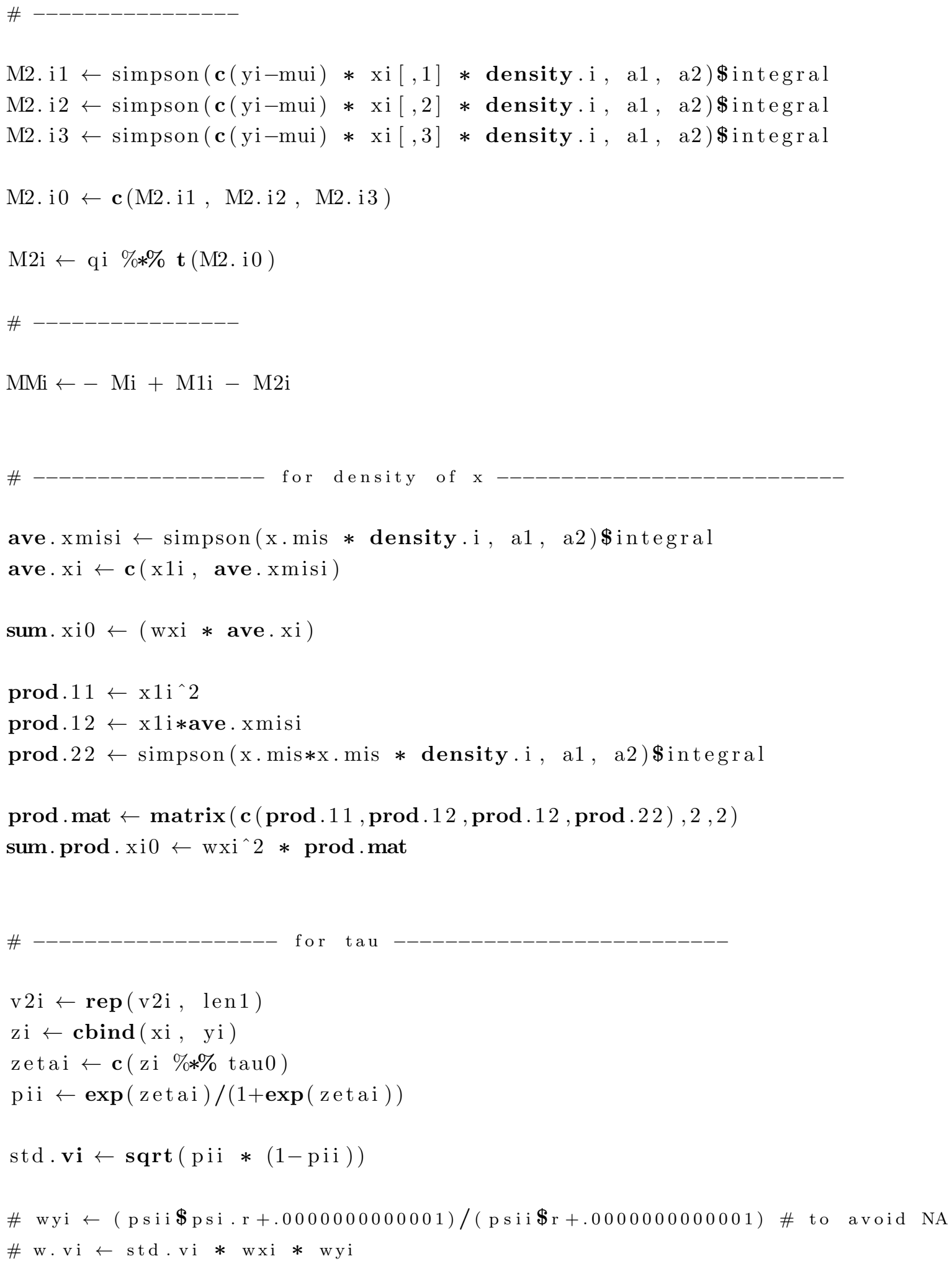




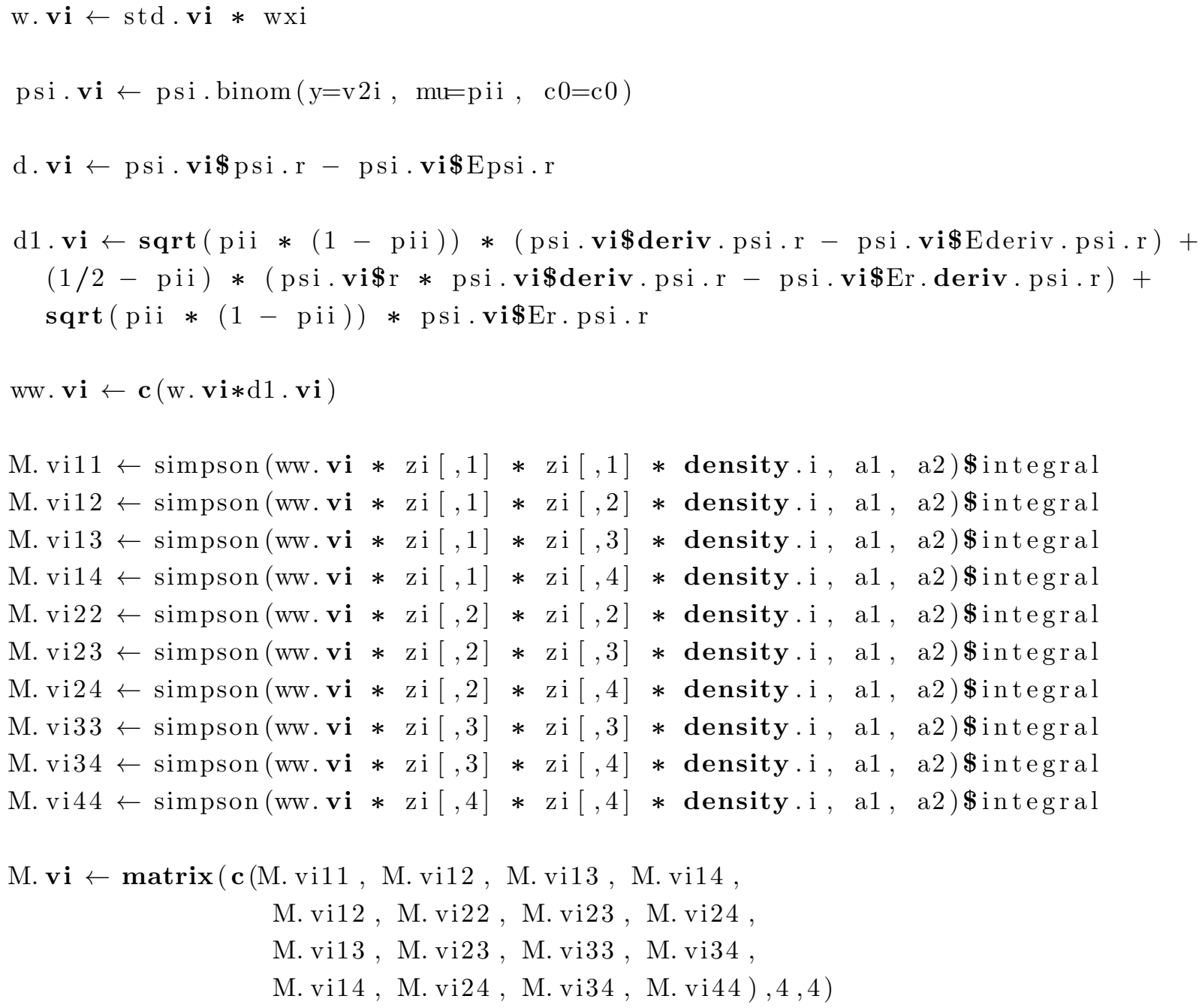




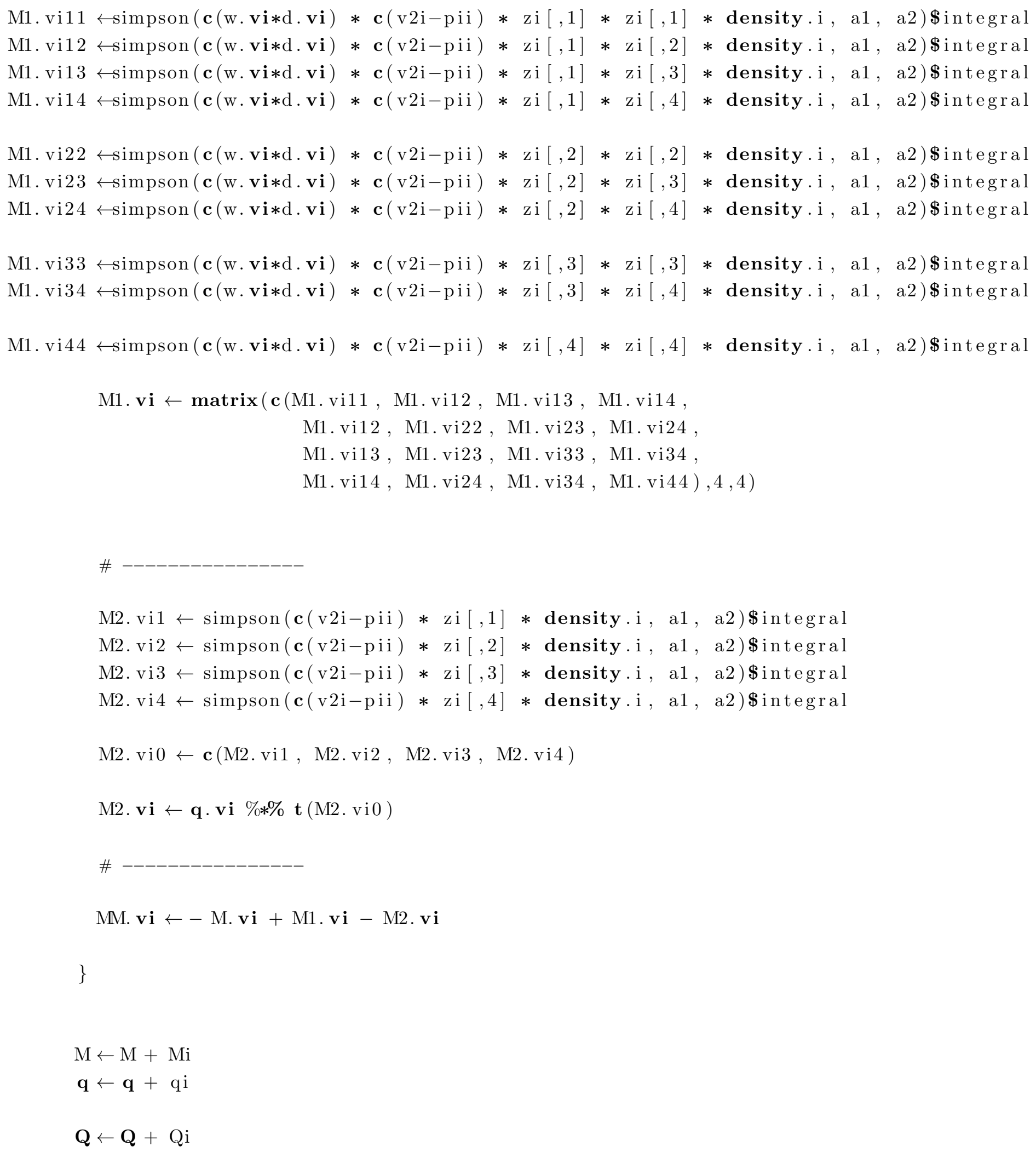




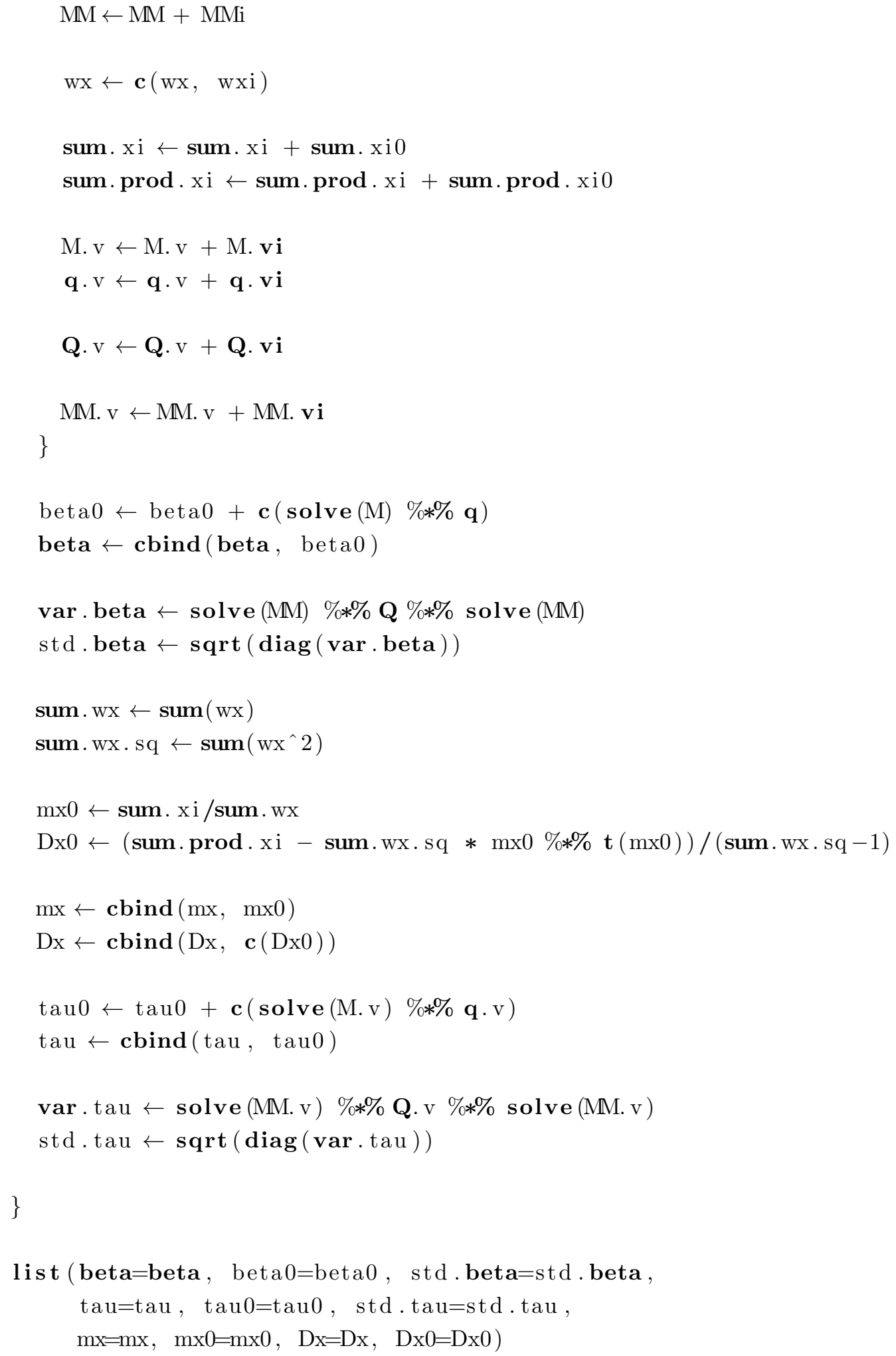


fit $1 \leftarrow \operatorname{rglmpois} . \operatorname{exact}(\mathrm{dat}=\mathrm{data} 0$, beta $0=\mathbf{c}(.5,1, .5), \operatorname{tau} 0=\mathbf{c}(-2, .5, .5, .25)$, $\operatorname{mx} 0=\mathbf{c}(.5, .5), \quad \mathrm{Dx} 0=\operatorname{matrix}(\mathbf{c}(.5, .1, .1, .5), 2,2), \quad \mathrm{c} 0=999$, gam $=0$, it ter $=20)$

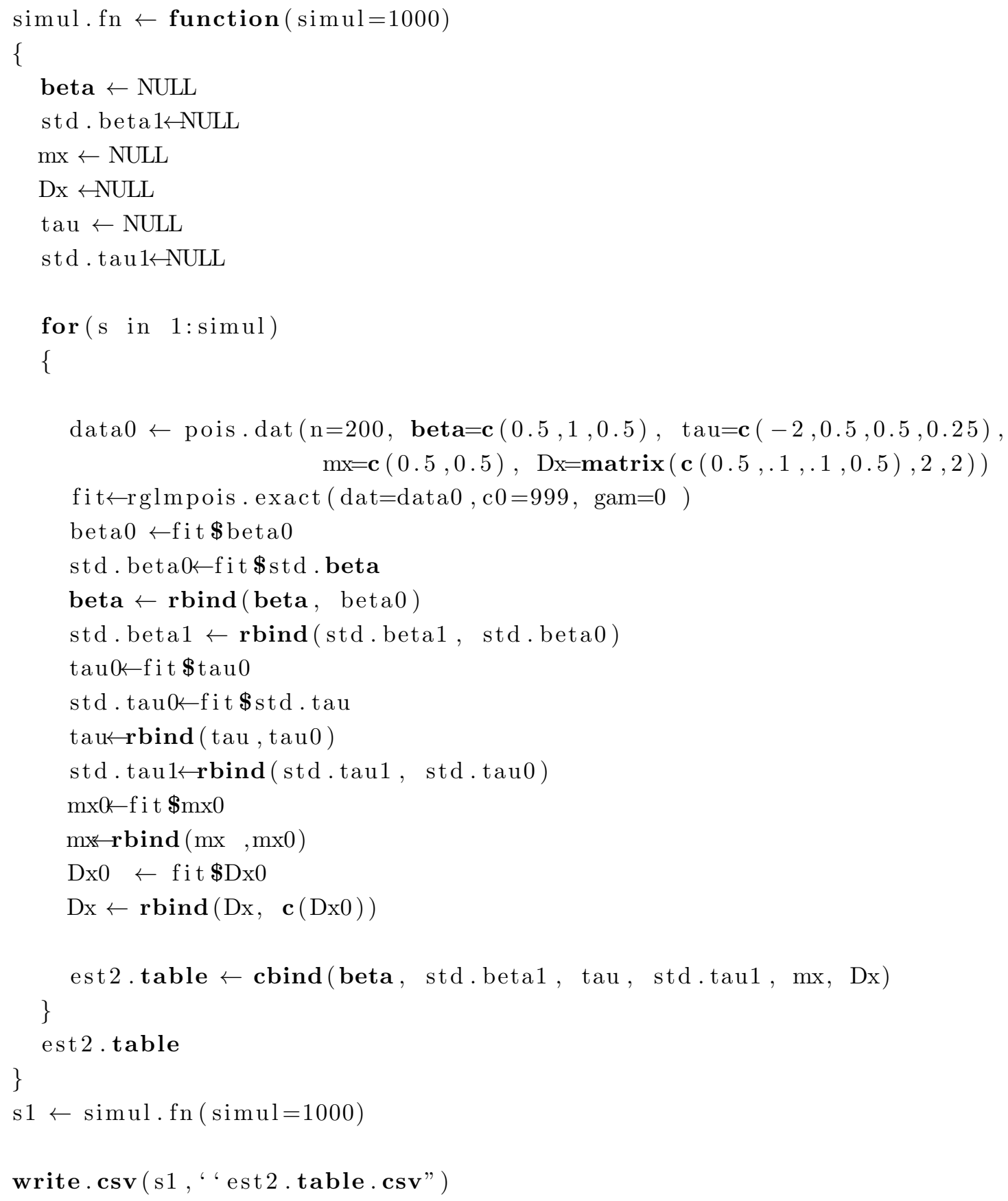

